\title{
On the two-variable Iwasawa main conjecture
}

\author{
Tadashi Ochiai
}

\begin{abstract}
This paper is a continuation of the author's previous work, where we studied one of the inequalities between the characteristic ideal of the Selmer group and the ideal of the $p$-adic $L$-function predicted by the two-variable Iwasawa main conjecture for a nearly ordinary Hida deformation $\mathcal{T}$. In this paper, we study several properties of the Selmer group and the $p$-adic $L$-function solving some of the open questions raised in the author's previous work. As applications, we have an infinite family of elliptic cuspforms where the cyclotomic Iwasawa main conjecture holds for every cuspform in the family.
\end{abstract}

\section{Contents}

1 Introduction

2 Overview of our program in the case of nearly ordinary Hida deformations

3 Local monodromy on $\mathbb{T}_{\mathcal{F}}^{\text {ord }}$

4 Selmer groups for Galois deformations

4.1 Selmer groups over discrete valuation rings . . . . . . . . . . . . . 1167

4.2 Selmer groups over one-variable Iwasawa algebras . . . . . . . . . . . . . 1169

4.3 Selmer groups over the two-variable Iwasawa algebra . . . . . . . . . . . 1174

4.4 Surjectivity of localization maps . . . . . . . . . . . . . . . 1175

5 Control theorem for Greenberg's Selmer groups $\quad 1177$

5.1 From two variables to one variable . . . . . . . . . . . . . . . 1177

5.2 From one variable to the discrete valuation case . . . . . . . . . . . . . 1179

6 Two-variable $p$-adic $L$-function $\quad 1180$

6.1 Review of the work of $[\mathrm{Och} 03] \ldots \ldots \ldots \ldots$. . . . . . . . 1181

6.2 p-adic error terms at weight two . . . . . . . . . . . . . 1182

6.3 Beilinson-Kato element . . . . . . . . . . . . . . 1183

$7 \begin{array}{lll} & \text { IMCs for various specializations of } \mathcal{T} & 1187\end{array}$

8 Pseudo-null submodule $\quad 1192$

9 Examples 1195

9.1 IMC for Ramanujan's cuspform . . . . . . . . . . . . . . . . . . . . 1195

9.2 Ramanujan's cuspform at $p=11 \ldots \ldots \ldots$. . . . . . . . . 1197

$\begin{array}{lr}\text { References } & 1199\end{array}$

\section{Introduction}

This paper is a continuation of the work on the Iwasawa theory for two-variable nearly ordinary modular Galois deformations [Och01, Och03, Och05]. By improving some of the author's previous

Received 19 April 2005, accepted in final form 4 April 2006.

2000 Mathematics Subject Classification 11F33, 11F80, 11G40, 11R23, 11R34.

Keywords: Iwasawa main conjecture, Selmer group, $p$-adic $L$-function, Hida theory.

The author is supported by the Japan Society for the Promotion of Science.

This journal is (c) Foundation Compositio Mathematica 2006. 


\section{T. OCHIAI}

results, we establish one of the inequalities of the two-variable Iwasawa main conjecture (IMC) and its corollaries in this paper.

In this section, we present a very brief plan of our research including future perspectives before stating a detailed description of our theory and our results in the next section. Our interest is to formulate a generalization of the IMC and to solve it for plenty of examples. The setting we consider is a pair $(\mathcal{T}, P)$ as follows.

(1) $\mathcal{T}$ is a free $\mathcal{R}$-module of finite rank, where $\mathcal{R}$ is a local Noetherian algebra which is finite flat over a several-variable Iwasawa algebra $\mathbb{Z}_{p}\left[\left[X_{1}, \ldots, X_{g}\right]\right]$ with a fixed prime $p$.

(2) There exists a finite number of primes $\Sigma$ containing $\{p, \infty\}$ such that we have a continuous $\mathcal{R}$-linear action of $G_{\mathbb{Q}}=\operatorname{Gal}(\overline{\mathbb{Q}} / \mathbb{Q})$ on $\mathcal{T}$ which is unramified outside $\Sigma$.

(3) There exists a certain dense subset $P$ of $\operatorname{Spec}(\mathcal{R})$ which consists of the kernels of various specializations $\mathcal{R} \longrightarrow \overline{\mathbb{Q}}$ such that the specialization of $\mathcal{T}$ at $\kappa \in P$ is a $G_{\mathbb{Q}^{-}}$-stable lattice of the $p$-adic étale realization of $M_{\kappa}$ for a critical motive $M_{\kappa}$.

We call a pair $(\mathcal{T}, P)$ satisfying the above conditions a geometric pair. Given a geometric pair $(\mathcal{T}, P)$, we would like to study the following objects:

(A) the analytic $p$-adic $L$-function $L_{p}^{\text {anal }}(\mathcal{T}) \in \operatorname{Frac}(\mathcal{R})$ such that the evaluation at every $\kappa \in P$ of $L_{p}^{\text {anal }}(\mathcal{T})$ is related to the special value of the Hasse-Weil $L$-function $L\left(M_{\kappa}, 0\right)$;

(B) the algebraic $p$-adic $L$-function $L_{p}^{\operatorname{alg}}(\mathcal{T}) \in \operatorname{Frac}(\mathcal{R})$, which is defined to be a generator of the characteristic ideal of the Pontryagin dual $\operatorname{Sel}_{\mathcal{T}}^{\vee}$ of the Selmer group $\operatorname{Sel}_{\mathcal{T}}$.

The existence of such $L_{p}^{\mathrm{anal}}(\mathcal{T})$ and $L_{p}^{\mathrm{alg}}(\mathcal{T})$ as non-zero elements in $\operatorname{Frac}(\mathcal{R})$ is expected only when $(\mathcal{T}, P)$ satisfies a certain local condition called 'nearly ordinary' or 'admissible' as well as certain minor extra conditions. We omit the precise definition of the notion 'nearly ordinary' or 'admissible' and we only refer the reader to [Gre94] (the notion called Panchishkin type in [Gre94] is very close to 'admissible').

(C) Assuming admissibility of $(\mathcal{T}, P)$, we expect equality $\left(L_{p}^{\text {alg }}(\mathcal{T})\right)=\left(L_{p}^{\text {anal }}(\mathcal{T})\right)$ of fractional ideals of $\mathcal{R}$, once they are well-defined as non-zero ideals in $\mathcal{R}$. This will be a generalization of the IMC which contains almost all known examples of the IMC.

A certain class of deformations called the cyclotomic deformations are rather classical examples in our program of 'the Iwasawa theory for Galois deformations'. Let $\mathcal{O}$ be a discrete valuation ring which is finite flat over $\mathbb{Z}_{p}$ and let $T$ be a free $\mathcal{O}$-module of finite rank $d$ which is the $p$-adic étale realization of a critical motive over $\mathbb{Q}$. Let $\Gamma$ be the Galois group $\operatorname{Gal}\left(\mathbb{Q}_{\infty} / \mathbb{Q}\right)$ of the cyclotomic $\mathbb{Z}_{p^{-}}$ extension $\mathbb{Q}_{\infty} / \mathbb{Q}$ of the rational number field $\mathbb{Q}$. We denote by $\tilde{\chi}$ the universal cyclotomic character $G_{\mathbb{Q}} \rightarrow \Gamma \hookrightarrow \mathbb{Z}_{p}[[\Gamma]]^{\times}$and $\mathbb{Z}_{p}[[\Gamma]](\tilde{\chi})$ is a free $\mathbb{Z}_{p}[[\Gamma]]$-module of rank one on which $G_{\mathbb{Q}}$ acts via the character $\tilde{\chi}$. If we consider $\mathcal{T}=T \widehat{\otimes}_{\mathbb{Z}_{p}} \mathbb{Z}_{p}[[\Gamma]](\tilde{\chi})$ and $\mathcal{R}=\mathbb{Z}_{p}[[\Gamma]], \mathcal{T}$ is free of rank $d$ over $\mathcal{R}$ and $G_{\mathbb{Q}}$ acts on $\mathcal{T}$ diagonally. It is known by Serre that $\mathbb{Z}_{p}[[\Gamma]]$ is non-canonically isomorphic to $\mathbb{Z}_{p}[[X]]$. Thus, by choosing $P$ to be a suitable set of primes in $\mathcal{R}=\mathbb{Z}_{p}[[\Gamma]]$ corresponding to Dirichlet characters of $p$-power conductor, $(\mathcal{T}, P)$ satisfies our previous conditions. If $T$ is ordinary at $p$ in the sense of Greenberg [Gre87], $(\mathcal{T}, P)$ is 'admissible'. When $T$ is a representation of rank one associated to a Dirichlet motive, study of (A), (B) and (C) is nothing but the Iwasawa theory for ideal class groups on a cyclotomic tower initiated by Iwasawa. When $T$ is a representation of rank two isomorphic to the $p$-adic Tate module $T_{p} E$ of an elliptic curve $E$ over $\mathbb{Q}$ ordinary at $p$, study of (A), (B) and (C) corresponds to the study of the Iwasawa theory for elliptic curves proposed by Mazur and developed by various people (cf. [Gre99]). The conjecture for existence of the analytic $p$-adic $L$-function (the subject for (A)) for all ordinary cyclotomic deformations is well-formulated by [CP89] and it is solved for class groups, elliptic modular forms and a few other examples. On the 


\section{ON THE TWO-VARIABLE IWASAWA MAIN CONJECTURE}

other hand, the Selmer group $\operatorname{Sel}_{\mathcal{T}}$ related to the algebraic $p$-adic $L$-function (the subject for (B)) has been defined and actively studied by Greenberg [Gre87] for ordinary cyclotomic deformations. $\operatorname{Sel}_{\mathcal{T}}$ is defined to be a subgroup of a Galois cohomology with coefficient $\mathcal{A}=\mathcal{T} \otimes_{\mathcal{R}} \mathcal{R}^{\vee}$. In order that the algebraic $p$-adic $L$-function is non-trivial, we need to assume the conjecture that $\operatorname{Sel}_{\mathcal{T}}^{\vee}$ is a torsion $\mathcal{R}$-module which is proved for only a few examples of critical motives.

To summarize, we can say that the conjectural framework of the Iwasawa main conjecture for ordinary cyclotomic deformations is rather established, although it is far from being proved except for a few examples.

Now, we remark that the category of ordinary cyclotomic deformations is naturally extended to the category of admissible geometric pairs as follows:

$\{$ ordinary cyclotomic deformations $\} \subset\{$ admissible geometric pairs $\}$.

A generalization of Iwasawa theory to the category of all admissible pairs was first proposed by Greenberg [Gre94]. However, in spite of work by several others who follow it, even a conjectural framework has not been well-understood because of the many new phenomena which do not happen for cyclotomic deformations. The two-variable nearly ordinary modular deformation constructed by Hida is an admissible geometric pair which is not an ordinary cyclotomic deformation. It seems to be the first step which we have to study toward our goal to understand the framework of the generalized Iwasawa theory which covers all admissible geometric pairs.

The work in this paper has thus been motivated. We close this section by emphasizing several important contributions made in this paper and motivating the inclusion of some technical sections such as $\S \S 2,3$ and 6 , so that the reader will not mistake the paper for a long review of previous results.

(1) For analytic $p$-adic $L$-functions in our two-variable deformation (the subject for (A)), there are several different constructions by Kitagawa, Greenberg and Stevens, Ochiai, Panchishkin and Fukaya. The first two are based on the method of modular symbols and the last three are based on the use of the Eisenstein series and the method of Shimura and Rankin-Selberg. The analytic $p$-adic $L$-function in this case is roughly a function in two variables $j, k$ as below:

$$
\frac{L_{p}^{\mathrm{anal}}(\mathcal{T})(j, k)}{C_{k, p}^{+}}=\left(\frac{p^{(j-1)}}{a_{p}\left(f_{k}\right)}\right)^{c(j)}\left(1-\frac{\omega^{1-j}(p) p^{c(j)}}{a_{p}\left(f_{k}\right)}\right) G\left(\omega^{1-j}\right) \frac{L\left(f_{k}, \omega^{1-j}, j\right)}{(2 \pi \sqrt{-1})^{j} \Omega_{k, \infty}^{+}}
$$

where $c(j)=\operatorname{ord}_{p} \operatorname{Cond}\left(\omega^{1-j}\right)$. Although we cannot explain all the notation in the above equation, which will be given in $\S \S 2$ and 6 , we insist that the differences of several constructions listed at the beginning of this paragraph appear only in the difference of $p$-adic error terms $C_{k, p}^{+}$and complex periods $\Omega_{k, \infty}^{+}$when $k$ varies. In the method of modular symbols, $\Omega_{k, \infty}^{+}$is a period integral associated to the modular form $f_{k}$ depending on a certain (non-canonical) choice of a basis of the module of modular symbols. In the latter method, $\Omega_{k, \infty}^{+}$is replaced by the period in the sense of Shimura which is obtained by a Rankin-Selberg integral depending on a choice of an Eisenstein series.

To compare these two essentially different constructions and to choose the best candidate for (A) seems to be an important problem to establish the Iwasawa theory in this particular case and it also seems important for our future perspectives. However, such a problem has not been studied previously. As we will see in $\S 6$, the best candidate for (A) seems to be that constructed by Kitagawa, since it is a good match for the generalized Birch and Swinnerton-Dyer conjecture. On the other hand, the construction of the analytic $p$-adic $L$-function using the method of Shimura and Rankin-Selberg has the advantage that it is more easily related to the algebraic $p$-adic $L$-function by applying the Euler system theory thanks to Kato's construction [Kat04]. 


\section{T. OCHIAI}

We have obtained an inequality as follows by combining [Och03] and [Och05]:

$$
\left(L_{p}^{\mathrm{O}}(\mathcal{T})\right) \subset\left(L_{p}^{\operatorname{alg}}(\mathcal{T})\right),
$$

where $L_{p}^{\mathrm{O}}(\mathcal{T})$ is an analytic $p$-adic $L$-function constructed in [Och03] from the Euler system obtained by Beilinson-Kato elements and $L_{p}^{\mathrm{O}}(\mathcal{T})$ is essentially the same as those constructed by Panchishkin and Fukaya. Owing to the difference of terms $C_{k, p}^{+}$and $\Omega_{k, \infty}^{+}$by multiplication of algebraic numbers at each $k$ of the interpolation $(1)$, we have a relation $\left(L_{p}^{\mathrm{O}}(\mathcal{T})\right) \subset\left(L_{p}^{\mathrm{Ki}}(\mathcal{T})\right)$ and these two can be different in general. Our observation through our work is that we can modify our Euler system by taking a certain linear combination and a certain projective limit so that the $p$-adic $L$-function obtained from the modified Euler system coincides with $L_{p}^{\mathrm{Ki}}(\mathcal{T})$ (cf. $\S 6$ ). By applying our Euler system theory [Och05] to the modified Euler system, we obtain the inequality as follows which improves $(2)$ :

$$
\left(L_{p}^{\mathrm{Ki}}(\mathcal{T})\right) \subset\left(L_{p}^{\operatorname{alg}}(\mathcal{T})\right) .
$$

For this reason, we have to give a rather detailed description of the theory, a part of which might seem to be a review of our previous results contrary to our intension.

(2) For algebraic $p$-adic $L$-functions in our two-variable deformation (the subject for (B)), we have a similar problem to compare different Selmer groups such as those defined by Greenberg or Bloch-Kato. For cyclotomic deformations, the difference is rather well-known. In the case of the cyclotomic deformation of an ordinary elliptic curve $E$, the algebraic $p$-adic $L$-function defined by Greenberg's Selmer group is equal to that defined by Bloch-Kato's Selmer group (cf. [BK90, $\S 4]$ ) when $E$ is not a Tate curve at $p$ and these two $L$-functions differ by a factor which comes from 'trivial zero' when $E$ is a Tate curve at $p$. Since the analytic $p$-adic $L$-function of a Tate curve also has a factor of 'trivial zero', Greenberg's Selmer group is the one according to the analytic $p$-adic $L$-function in view of the IMC (the subject for $(\mathrm{C})$ ).

Not only is it useful for finding the best candidate for a Selmer group in accordance with the Iwasawa main conjecture; the comparison of different Selmer groups seems to be an important problem in itself. Thus, we compare two different Selmer groups defined by Greenberg's method and by Bloch-Kato's method for large Galois representations over the two-variable nearly ordinary algebra in $\S 4$ of this paper.

We believe that we have taken a step forward to 'the Iwasawa theory for Galois deformations' through our detailed study in this paper for two-variable nearly ordinary deformations.

\section{Notation}

For an integer $r$, we denote by $\mu_{r}$ the group of $r$ th roots of unity and denote by $\mathbb{Q}\left(\mu_{r}\right)$ the field obtained by adjoining $\mu_{r}$ to the rational number field $\mathbb{Q}$. We often denote by $\mathbb{Q}\left(\mu_{p^{\infty}}\right)$ the field obtained by adjoining all $p$-power roots of unity to the rational number field $\mathbb{Q}$. For any Galois extension $L / \mathbb{Q}$ and a prime number $q$ which is unramified in $L / \mathbb{Q}$, we denote by $\operatorname{Frob}_{q} \in \operatorname{Gal}(L / \mathbb{Q})$ (respectively $\varphi_{q} \in \operatorname{Gal}(L / \mathbb{Q})$ ) (a conjugate class of) a geometric (respectively arithmetic) Frobenius element at $q$.

\section{Overview of our program in the case of nearly ordinary Hida deformations}

In this section, we introduce our results for the Iwasawa theory on Hida deformations obtained in [Och03] and [Och05]. We will also give a slight modification (see Theorem 2 and Remark 2.5) of our Euler system theory to give an application in $\S 9$.

To introduce our results, let us recall briefly Hida's nearly ordinary modular deformations. 


\section{ON THE TWO-VARIABLE IWASAWA MAIN CONJECTURE}

We fix a prime number $p \geqslant 3$ and a norm compatible system $\left\{\zeta_{p^{n}}\right\}_{n \geqslant 1}$ of primitive $p^{n}$ th roots of unity throughout the paper. Let $\Gamma$ be the Galois group $\operatorname{Gal}\left(\mathbb{Q}_{\infty} / \mathbb{Q}\right)$ of the cyclotomic $\mathbb{Z}_{p}$-extension $\mathbb{Q}_{\infty} / \mathbb{Q}$ of the rational number field $\mathbb{Q}$. We denote by $\Gamma^{\prime}$ the group of diamond operators for the tower of modular curves $\left\{Y_{1}\left(p^{t}\right)\right\}_{t \geqslant 1}$. We have the canonical isomorphisms:

$$
\Gamma \underset{\chi}{\stackrel{\sim}{\longrightarrow}} 1+p \mathbb{Z}_{p} \subset \mathbb{Z}_{p}^{\times}, \quad \Gamma^{\prime} \underset{\kappa}{\stackrel{\sim}{\longrightarrow}} 1+p \mathbb{Z}_{p} \subset \mathbb{Z}_{p}^{\times} .
$$

Fix a topological generator $\gamma$ (respectively $\gamma^{\prime}$ ) of $\Gamma$ (respectively $\Gamma^{\prime}$ ). For later convenience, we choose $\gamma$ and $\gamma^{\prime}$ so that $\chi(\gamma)=\kappa\left(\gamma^{\prime}\right)$. From now on, we fix an embedding of an algebraic closure $\overline{\mathbb{Q}}$ into the field $\mathbb{C}$ of complex numbers and an embedding of $\overline{\mathbb{Q}}$ into a fixed algebraic closure $\overline{\mathbb{Q}}_{p}$ of the field $\mathbb{Q}_{p}$ of $p$-adic numbers simultaneously. We also fix a natural number $N$ prime to $p$.

Let $\mathbb{H}_{\mathcal{F}}^{\text {ord }}$ be the quotient of the universal ordinary Hecke algebra $\mathbb{H}_{N p^{\infty}}^{\text {ord }}$ with tame conductor $N$, which corresponds to a certain $\Lambda$-adic eigen-cuspform $\mathcal{F}$. The algebra $\mathbb{H}_{\mathcal{F}}^{\text {ord }}$ is a local domain, finite flat over $\mathbb{Z}_{p}\left[\left[\Gamma^{\prime}\right]\right]$. Then (the $\mathcal{F}$-component of) Hida's nearly ordinary Hecke algebra $\mathbb{H}_{\mathcal{F}}^{\text {n.o }}$ is defined to be the formal tensor product of $\mathbb{H}_{\mathcal{F}}^{\text {ord }}$ and the cyclotomic Iwasawa algebra $\mathbb{Z}_{p}[[\Gamma]]$. By this, $\mathbb{H}_{\mathcal{F}}^{\text {n.o }}$ is isomorphic to $\mathbb{H}_{\mathcal{F}}^{\text {ord }}[[\Gamma]]$ and is a local domain, finite flat over $\mathbb{Z}_{p}\left[\left[\Gamma \times \Gamma^{\prime}\right]\right]$. Let $\Sigma$ be the finite set of places of $\mathbb{Q}$ consisting of $\{\infty\}$ and the primes dividing $N p$. In his celebrated paper [Hid86b], Hida constructs a large continuous Galois representation $\rho: G_{\mathbb{Q}} \longrightarrow \operatorname{Aut}_{\mathbb{H}_{\mathcal{F}}^{\text {n.o }}}\left(\mathcal{T}_{\mathcal{F}}^{(0)}\right)$ unramified outside of $\Sigma$, where $\mathcal{T}_{\mathcal{F}}^{(0)}$ is a finitely generated torsion-free module of generic rank two over $\mathbb{H}_{\mathcal{F}}^{\text {n.o }}$. The representation $\mathcal{T}_{\mathcal{F}}^{(0)}$ is presented as $\mathbb{T}_{\mathcal{F}}^{\text {ord }} \widehat{\otimes} \mathbb{Z}_{p}[[\Gamma]](\widetilde{\chi})$, where $\mathbb{T}_{\mathcal{F}}^{\text {ord }}$ is a finitely generated torsionfree module of generic rank two over $\mathbb{H}_{\mathcal{F}}^{\text {ord }}$ with continuous $G_{\mathbb{Q}}$-action. The trace of the Frobenius element $\operatorname{Fr}_{l} \in G_{\mathbb{Q}}$ acting on $\mathbb{T}_{\mathcal{F}}^{\text {ord }}$ is equal to the Fourier coefficient $A_{l}(\mathcal{F})$ of $\mathcal{F}$ for every prime $l \notin \Sigma$. Let $\mathfrak{M}$ be the maximal ideal of $\mathbb{H}_{\mathcal{F}}^{\text {n.o }}$ and let $\mathbb{F}$ be a finite residue field $\mathbb{H}_{\mathcal{F}}^{\text {n.o }} / \mathfrak{M}$. The residual representation of $\mathcal{T}_{\mathcal{F}}^{(0)}$ is defined to be a rank-two $\mathbb{F}$-module with semi-simple $G_{\mathbb{Q}^{-a c t i o n}}$ where the trace of $\operatorname{Fr}_{l}$ is congruent to $A_{l}(\mathcal{F})$ modulo $\mathfrak{M}$ for every prime $l \notin \Sigma$. Such residual representation of $\mathcal{T}_{\mathcal{F}}^{(0)}$ is always known to exist by Hida (cf. [MW86, $\S 9$ ]) and is unique up to isomorphism by the Chebotarev density theorem. Throughout the paper, we always assume the following condition unless otherwise stated.

CONDition (Ir). The residual representation of $\mathcal{T}_{\mathcal{F}}^{(0)}$ is an irreducible $G_{\mathbb{Q}}$-module.

Condition (Ir) implies that $\mathbb{T}_{\mathcal{F}}^{\text {ord }}$ (respectively $\mathcal{T}_{\mathcal{F}}^{(0)}$ ) is free of rank two over $\mathbb{H}_{\mathcal{F}}^{\text {ord }}$ (respectively $\left.\mathbb{H}_{\mathcal{F}}^{\text {n.o }}\right)$. Let us recall the following definition.

Definition 2.1. Let $w$ be an integer. A point $\mathfrak{I} \in \operatorname{Hom}_{\mathbb{Z}_{p}}\left(\mathbb{H}_{\mathcal{F}}^{\text {ord }}, \overline{\mathbb{Q}}_{p}\right)$ is called an arithmetic point of weight $w$ if there exists an open subgroup $U$ of $\Gamma^{\prime}$ such that the restriction $\left.\mathfrak{I}\right|_{U}: U \hookrightarrow \mathbb{Z}_{p}\left[\left[\Gamma^{\prime}\right]\right]^{\times} \hookrightarrow$ $\left(\mathbb{H}_{\mathcal{F}}^{\text {ord }}\right)^{\times} \stackrel{\mathfrak{I}}{\longrightarrow} \overline{\mathbb{Q}}_{p}^{\times}$sends $u$ to $\kappa^{w}(u)$ for any $u \in U$. We denote by $\mathfrak{X}_{\text {arith }}\left(\mathbb{H}_{\mathcal{F}}^{\text {ord }}\right)$ the set of arithmetic points of $\mathbb{H}_{\mathcal{F}}^{\text {ord }}$. For an arithmetic point $\mathfrak{I}$ of $\mathbb{H}_{\mathcal{F}}^{\text {ord }}$, we will denote by $w(\mathfrak{I})$ the weight of $\mathfrak{I}$. We define a subset $\mathfrak{X}_{\text {arith }}\left(\mathbb{H}_{\mathcal{F}}^{\text {ord }}\right)_{\geqslant 0} \subset \mathfrak{X}_{\text {arith }}\left(\mathbb{H}_{\mathcal{F}}^{\text {ord }}\right)$ to be $\mathfrak{X}_{\text {arith }}\left(\mathbb{H}_{\mathcal{F}}^{\text {ord }}\right)_{\geqslant 0}=\left\{\mathfrak{I} \in \mathfrak{X}_{\text {arith }}\left(\mathbb{H}_{\mathcal{F}}^{\text {ord }}\right) \mid w(\mathfrak{I}) \geqslant 0\right\}$.

We briefly recall the properties of $\mathcal{T}_{\mathcal{F}}^{(0)}$ (cf. [Hid86b, Wil88]).

\section{Basic property of nearly ordinary Hida deformations $\mathcal{T}_{\mathcal{F}}^{(0)}$}

Assume Condition (Ir). The deformation $\mathcal{T}_{\mathcal{F}}^{(0)}$ (respectively $\mathbb{T}_{\mathcal{F}}^{\text {ord }}$ ) has the following properties.

(1) For each $\mathfrak{I} \in \mathfrak{X}_{\text {arith }}\left(\mathbb{H}_{\mathcal{F}}^{\text {ord }}\right)_{\geqslant 0}$, there exists a normalized eigen-cuspform $f_{\mathfrak{I}}$ of weight $w(\mathfrak{I})+$ 2 and the quotient $\mathbb{T}^{\text {ord }} / \operatorname{Ker}(\mathfrak{I}) \mathbb{T}_{\mathcal{F}}^{\text {ord }} \cong \mathcal{O}_{\mathfrak{I}}^{\oplus 2}$ with $\mathcal{O}_{\mathfrak{I}}:=\mathbb{H}_{\mathcal{F}}^{\text {ord }} / \operatorname{Ker}(\mathfrak{I})$ is isomorphic to $T_{f_{\mathfrak{I}}}$, where $T_{f_{\mathfrak{I}}}$ is the unique lattice of Deligne's Galois representation associated to $f_{\mathfrak{I}}$ (cf. [Del69]). Thus, $\mathcal{T}_{\mathcal{F}}^{(0)} /\left(\operatorname{Ker}(\mathfrak{I}), \gamma-\chi^{j}(\gamma)\right) \mathcal{T}_{\mathcal{F}}^{(0)}$ is isomorphic to $T_{f_{\mathfrak{I}}} \otimes \chi^{j}$ for each $j \in \mathbb{Z}$ and each $\mathfrak{I} \in$ $\mathfrak{X}_{\text {arith }}\left(\mathbb{H}_{\mathcal{F}}^{\text {ord }}\right) \geqslant 0$. 


\section{T. OCHIAI}

(2) As a representation of the decomposition group $G_{\mathbb{Q}_{p}} \subset G_{\mathbb{Q}}$ at $p, \mathcal{T}_{\mathcal{F}}^{(0)}$ has a filtration $0 \longrightarrow$ $\mathrm{F}^{+} \mathcal{T}_{\mathcal{F}}^{(0)} \longrightarrow \mathcal{T}_{\mathcal{F}}^{(0)} \longrightarrow \mathrm{F}^{-} \mathcal{T}_{\mathcal{F}}^{(0)} \longrightarrow 0$ such that the graded pieces $\mathrm{F}^{+} \mathcal{T}_{\mathcal{F}}^{(0)}$ and $\mathrm{F}^{-} \mathcal{T}_{\mathcal{F}}^{(0)}$ are free of rank one over $\mathbb{H}_{\mathcal{F}}^{\text {n.o }}$.

(3) Further, $\mathrm{F}^{+} \mathcal{T}_{\mathcal{F}}^{(0)}$ is isomorphic to $\mathbb{Z}_{p}[[\Gamma]](\widetilde{\chi}) \widehat{\otimes}_{\mathbb{Z}_{p}} \mathbb{H}_{\mathcal{F}}^{\text {ord }}(\widetilde{\alpha})$ as a $G_{\mathbb{Q}_{p}}$-module, where $\widetilde{\alpha}$ is an unramified character $G_{\mathbb{Q}_{p}} \longrightarrow\left(\mathbb{H}_{\mathcal{F}}^{\text {ord }}\right)^{\times}$such that $A_{p}(\mathcal{F})=\widetilde{\alpha}\left(\right.$ Frob $\left._{p}\right) \in \mathbb{H}_{\mathcal{F}}^{\text {ord }}$ satisfies an interpolation property $\mathfrak{I}\left(A_{p}(\mathcal{F})\right)=a_{p}\left(f_{\mathfrak{I}}\right)$ for each $\mathfrak{I} \in \mathfrak{X}_{\text {arith }}\left(\mathbb{H}_{\mathcal{F}}^{\text {ord }}\right)_{\geqslant 0}$ and $\mathbb{H}_{\mathcal{F}}^{\text {ord }}(\widetilde{\alpha})$ is a rank-one free $\mathbb{H}_{\mathcal{F}}^{\text {ord }}$ module on which $G_{\mathbb{Q}_{p}}$ acts via the character $\widetilde{\alpha}$.

Let $\omega$ be the Teichmüller character. We will study the twist $\mathcal{T}_{\mathcal{F}}^{(i)}=\mathcal{T}_{\mathcal{F}}^{(0)} \otimes \omega^{i}$ for a fixed integer $0 \leqslant i \leqslant p-2$, which we call a nearly ordinary deformation. From now on, we will denote $\mathcal{T}_{\mathcal{F}}^{(i)}$ by $\mathcal{T}$ if this causes no possibility of confusion. We would like to study 'the Iwasawa theory for $\mathcal{T}$ '. The space of $p$-adic characters of $\mathbb{H}_{\mathcal{F}}^{\text {n.o }}$ is naturally viewed as a rigid analytic space, finite flat over a twodimensional open unit ball in $\mathbb{C}_{p}^{2}$. Hence, $\mathcal{T}$ corresponds to a family of Galois representations over a two-dimensional rigid space. Each 'hypersurface' of the space of characters of $\mathbb{H}_{\mathcal{F}}^{\text {n.o }}$ is a rigid space of dimension one, which also interests us from a view point of 'the Iwasawa theory for deformation spaces'. Among infinitely many hypersurfaces, we study the following four types of hypersurfaces $\mathcal{T}_{J}=\mathcal{T} / J \mathcal{T}$ for height one primes $J$ of $\mathbb{H}_{\mathcal{F}}^{\text {n.o }}$ in particular.

(a) Cyclotomic deformations of ordinary cuspforms. We have $\mathcal{T}_{I}=T_{f_{\mathfrak{I}} \otimes \omega^{i}} \otimes_{\mathbb{Z}_{p}} \mathbb{Z}_{p}[[\Gamma]](\tilde{\chi})$ for a cuspform $f_{\mathfrak{I}} \otimes \omega^{i}$ of weight $k=w(\mathfrak{I})+2$, which is free of rank two over $\mathcal{O}_{\mathfrak{I}}[[\Gamma]]$. Here, $\mathfrak{I} \in \mathfrak{X}_{\text {arith }}\left(\mathbb{H}_{\mathcal{F}}^{\text {ord }}\right)_{\geqslant 0}$ and $I$ is a height-one ideal $\operatorname{Ker}(\mathfrak{I}) \mathbb{H}_{\mathcal{F}}^{\text {n.o }}$ of $\mathbb{H}_{\mathcal{F}}^{\text {n.o. }}$. This is the case called 'the cyclotomic deformation' and has been developed by many people since Mazur [Maz72] started the Iwasawa theory for the cyclotomic deformation of an ordinary elliptic curve (see, for example, [Gre87, Gre99, MTT86]).

(b) Ordinary deformation twisted by $\chi$. We have, $\mathcal{T}_{(\gamma-\chi(\gamma))}=\mathbb{T}_{\mathcal{F}}^{\text {ord }} \otimes \chi \omega^{i}$, which is free of rank two over $\mathbb{H}_{\mathcal{F}}^{\text {ord }}$. For each $\mathfrak{I} \in \mathfrak{X}_{\text {arith }}\left(\mathbb{H}_{\mathcal{F}}^{\text {ord }}\right)_{\geqslant 0}, \mathcal{T}_{(\gamma-\chi(\gamma))} / \operatorname{Ker}(\mathfrak{I}) \mathcal{T}_{(\gamma-\chi(\gamma))}$ is isomorphic to $T_{f_{\mathfrak{I}}} \otimes \chi \omega^{i}$. Hence, $\mathcal{T}_{(\gamma-\chi(\gamma))}$ is the interpolation of the $\mathbb{Z}_{p}(1)$-twists of the Galois representations for $f_{\mathfrak{I}} \otimes$ $\omega^{i-1}$ when $\mathfrak{I}$ varies in $\mathfrak{X}_{\text {arith }}\left(\mathbb{H}_{\mathcal{F}}^{\text {ord }}\right) \geqslant 0$.

(c) Ordinary deformation twisted by $\mathbb{Z}_{p}[[\Gamma]](\widetilde{\chi}) \otimes \chi$. We have $\mathcal{T}_{\left(\gamma-\kappa\left(\gamma^{\prime}\right) \gamma^{\prime}\right)}=\mathbb{T}_{\mathcal{F}}^{\text {ord }} \otimes_{\mathbb{Z}_{p}\left[\left[\Gamma^{\prime}\right]\right]} \mathbb{Z}_{p}[[\Gamma]](\widetilde{\chi}) \otimes$ $\chi \omega^{i}$, which is free of rank two over $\mathbb{H}_{\mathcal{F}}^{\text {ord }}$. Note that $\mathcal{T}=\mathbb{T}_{\mathcal{F}}^{\text {ord }} \widehat{\otimes}_{\mathbb{Z}_{p}} \mathbb{Z}_{p}[[\Gamma]](\tilde{\chi}) \otimes \omega^{i}$ and that the tensor product is taken through the canonical isomorphism $\Gamma \stackrel{\sim}{\longrightarrow} \Gamma^{\prime}$. For each $\mathfrak{I} \in \mathfrak{X}_{\text {arith }}\left(\mathbb{H}_{\mathcal{F}}^{\text {ord }}\right)_{\geqslant 0}$, $\mathcal{T}_{\left(\gamma-\kappa\left(\gamma^{\prime}\right) \gamma^{\prime}\right)} / \operatorname{Ker}(\mathfrak{I}) \mathcal{T}_{\left(\gamma-\kappa\left(\gamma^{\prime}\right) \gamma^{\prime}\right)}$ is isomorphic to $T_{f_{\mathfrak{I}}} \otimes \chi^{w(\mathfrak{I})+1} \omega^{i}$. Hence, $\mathcal{T}_{\left(\gamma-\kappa\left(\gamma^{\prime}\right) \gamma^{\prime}\right)}$ is the interpolation of $\mathbb{Z}(1)^{\otimes w(\mathfrak{I})+1}$-twists of the Galois representations of $f_{\mathfrak{I}} \otimes w^{i-1-w(\mathfrak{I})}$ when $\mathfrak{I}$ varies in $\mathfrak{X}_{\text {arith }}\left(\mathbb{H}_{\mathcal{F}}^{\text {ord }}\right) \geqslant 0$.

(d) One-variable deformation at the diagonal line. We have

$$
\mathcal{T}_{\left(\gamma^{2}-\kappa^{2}\left(\gamma^{\prime}\right) \gamma^{\prime}\right)}=\mathbb{T}_{\mathcal{F}}^{\text {ord }} \otimes_{\mathbb{Z}_{p}\left[\left[\Gamma^{\prime}\right]\right]} \mathbb{Z}_{p}[[\Gamma]]\left(\widetilde{\chi}^{\frac{1}{2}}\right) \otimes \chi \omega^{i},
$$

which is free of rank two over $\mathbb{H}_{\mathcal{F}}^{\text {ord }}$. Similarly as above, $\mathcal{T}_{\left(\gamma^{2}-\kappa^{2}\left(\gamma^{\prime}\right) \gamma^{\prime}\right)}$ is the interpolation of $\mathbb{Z}(1)^{\otimes k(\mathfrak{I}) / 2}$-twists of the Galois representations of $f_{\mathfrak{I}} \otimes \omega^{i-k(\mathfrak{I}) / 2}$ when $\mathfrak{I}$ runs arithmetic points of $\mathbb{H}_{\mathcal{F}}^{\text {ord }}$ with $k(\mathfrak{I}) \in 2 \mathbb{Z}_{\geqslant 0}$, where $k(\mathfrak{I})=w(\mathfrak{I})+2$ is the weight of the cuspform $f_{\mathfrak{I}}$. Note that the representations with the above twist correspond to the special value of $L\left(f_{\mathfrak{I}} \otimes \omega^{i-k(\mathfrak{I}) / 2}, s\right)$ at the center of the functional equations when $\mathfrak{I}$ varies.

Some of the Iwasawa theoretic properties of $\mathcal{T}_{J}$ are deduced by the method of 'specialization' from those of $\mathcal{T}$ (see $\S 5$ for such a technique and also $\S 7$ for results and conjectures in these cases).

To introduce our main result, we recall the definition of the Euler system in our situation.

Definition 2.2. Let $\mathcal{T}^{*}(1)$ be the Kummer dual $\operatorname{Hom}_{\mathbb{H}_{\mathcal{F}}^{\text {n.o }}}\left(\mathcal{T}, \mathbb{H}_{\mathcal{F}}^{\text {n.o }}\right) \otimes_{\mathbb{Z}_{p}} \mathbb{Z}_{p}(1)$ of $\mathcal{T}$. An Euler system for $\mathcal{T}^{*}(1)$ is a collection of cohomology classes $\left\{\mathcal{C}(r) \in H^{1}\left(\mathbb{Q}\left(\mu_{r}\right), \mathcal{T}^{*}(1)\right)\right\}$ where $r$ runs the set of 


\section{ON THE TWO-VARIABLE IWASAWA MAIN CONJECTURE}

all square-free natural numbers prime to the tame conductor $N$ such that the following properties are satisfied.

(1) The element $\mathcal{C}(r)$ is unramified outside primes dividing $N$.

(2) The norm $\operatorname{Norm}_{\mathbb{Q}\left(\mu_{r q}\right) / \mathbb{Q}\left(\mu_{r}\right)} \mathcal{C}(r q)$ is equal to $P_{q}\left(\operatorname{Frob}_{q}\right) \mathcal{C}(r)$, where $P_{q}(X) \in \mathbb{H}_{\mathcal{F}}^{\text {n.o }}[X]$ is a polynomial $\operatorname{det}\left(1-\operatorname{Frob}_{q} X ; \mathcal{T}\right)$ and $\operatorname{Frob}_{q}$ is a (conjugacy class of) geometric Frobenius element at $q$ in the Galois group $\operatorname{Gal}\left(\mathbb{Q}\left(\mu_{r}\right) / \mathbb{Q}\right)$.

In the rest of this section, we will recall the results for the IMC for $\mathcal{T}$ and the specializations of $\mathcal{T}$. One of the results in this paper is Theorem 6.11 where we give an Euler system $\left\{\mathcal{Z}^{\mathrm{Ki}}(r) \in\right.$ $\left.H^{1}\left(\mathbb{Q}\left(\mu_{r}\right), \mathcal{T}^{*}(1)\right)\right\}$, which is an 'optimal' modification of Beilinson-Kato elements. On the other hand, we constructed the Coleman map $\Xi_{d}: H_{/ f}^{1}\left(\mathbb{Q}_{p}, \mathcal{T}^{*}(1)\right) \longrightarrow \mathbb{H}_{\mathcal{F}}^{\text {n.o }}($ cf. [Och03, Theorem 3.13]) which is recalled in this paper at Theorem 6.3. Combining these results, we have the following result (see $\S 6$ for some of the notation).

Theorem 1 (Corollary 6.17). Let $i$ be an integer such that $0 \leqslant i \leqslant p-2$. We assume Condition (Ir) for a nearly ordinary deformation $\mathcal{T}=\mathcal{T}_{\mathcal{F}}^{(i)}$. Assume also the following condition.

Condition (Nor). $\mathbb{H}_{\mathcal{F}}^{\text {n.o }}$ is integrally closed in its fraction field $\operatorname{Frac}\left(\mathbb{H}_{\mathcal{F}}^{\text {n.o }}\right)$.

We have the Euler system $\left\{\mathcal{Z}^{\mathrm{Ki}}(r) \in H^{1}\left(\mathbb{Q}\left(\mu_{r}\right), \mathcal{T}^{*}(1)\right)\right\}$ whose first layer $\mathcal{Z}^{\mathrm{Ki}}(1)$ satisfies the equality

$$
\operatorname{length}_{\mathbb{H}_{\mathcal{F}, \mathfrak{l}}^{\text {n.o }}}\left(H_{/ f}^{1}\left(\mathbb{Q}_{p}, \mathcal{T}^{*}(1)\right) / \operatorname{loc}_{/ f}\left(\mathcal{Z}^{\mathrm{Ki}}(1)\right) \mathbb{H}_{\mathcal{F}}^{\text {n.o }}\right)_{\mathfrak{l}}=\operatorname{ord}_{\mathfrak{l}}\left(L_{p}^{\mathrm{Ki}}(\mathcal{T})\right)
$$

for each height-one prime $\mathfrak{l} \subset \mathbb{H}_{\mathcal{F}}^{\text {n.o }}$, where $L_{p}^{\mathrm{Ki}}(\mathcal{T})$ is Kitagawa's two-variable p-adic L-function (see [Kit94] and also Theorem 6.7 for the interpolation property of Kitagawa's p-adic L-function) and $\mathbb{H}_{\mathcal{F}, \mathfrak{l}}^{\text {n.o }}$ is the discrete valuation ring obtained by localizing $\mathbb{H}_{\mathcal{F}}^{\text {n.o }}$ at $\mathfrak{l}$.

Remark 2.3. In the above theorem, the condition that $\mathbb{H}_{\mathcal{F}}^{\text {n.o }}$ is integrally closed is necessary only to assure that the image of $\Xi_{d}$ is contained in $\mathbb{H}_{\mathcal{F}}^{\text {n.o }}$. Without this condition, the image of $\Xi_{d}$ is in the fraction field $\operatorname{Frac}\left(\mathbb{H}_{\mathcal{F}}^{\text {n.o }}\right)$ of $\mathbb{H}_{\mathcal{F}}^{\text {n.o }}$ and the localization $\Xi_{d}(\mathcal{C})_{\mathfrak{l}}$ of $\Xi_{d}(\mathcal{C})$ is contained in $\mathbb{H}_{\mathcal{F}, \mathfrak{l}}^{\text {n.o }}$ for each height-one prime $\mathfrak{l} \subset \mathbb{H}_{\mathcal{F}}^{\text {n.o }}$. All interpolation properties as above hold without this condition (see the arguments in $[$ Och03, $\S 5]$ ).

On the other hand, we associate the Selmer group $\operatorname{Sel}_{\mathcal{T}}$ to $\mathcal{T}$. Let

$$
\mathcal{A}=\mathcal{T} \otimes_{\mathbb{H}_{\mathcal{F}}^{\text {noo }}} \operatorname{Hom}_{\mathbb{Z}_{p}}\left(\mathbb{H}_{\mathcal{F}}^{\text {n.o }}, \mathbb{Q}_{p} / \mathbb{Z}_{p}\right) \text {. }
$$

We define $\operatorname{Sel}_{\mathcal{T}}$ as a subgroup of $H^{1}\left(\mathbb{Q}_{\Sigma} / \mathbb{Q}, \mathcal{A}\right)$ (see $\S 4.3$ for the precise definition). The Pontryagin dual $\left(\operatorname{Sel}_{\mathcal{T}}\right)^{\vee}$ of $\operatorname{Sel}_{\mathcal{T}}$ is a finitely generated torsion $\mathbb{H}_{\mathcal{F}}^{\text {n.o }}$-module (cf. Proposition 4.9). We propose the following conjecture.

Conjecture 2.4 (Two-variable IMC). We assume Condition (Ir). We have the equality

$$
\operatorname{length}_{\mathbb{H}} \mathbb{H}_{\mathcal{F}, \mathfrak{l}}\left(\operatorname{Sel}_{\mathcal{T}}\right)_{\mathfrak{l}}^{\vee}=\operatorname{ord}_{\mathfrak{l}}\left(L_{p}^{\mathrm{Ki}}(\mathcal{T})\right)
$$

for each height-one prime $\mathfrak{l}$ of $\mathbb{H}_{\mathcal{F}}^{\text {n.o }}$.

In [Och05], we proved that the ideal associated to the (localization of the) Beilinson-Kato element for $\mathcal{T}$ is contained in the characteristic ideal of $\left(\operatorname{Sel}_{\mathcal{T}}\right)^{\vee}$. We restate the result, but with slight modification of the assumptions (see Remark 2.5 below).

Theorem 2. We assume that $\mathbb{H}_{\mathcal{F}}^{\text {n.o }}$ is isomorphic to a two-variable power series algebra $\mathcal{O}\left[\left[X_{1}, X_{2}\right]\right]$ over the ring of the integers $\mathcal{O}$ of a certain finite extension of $\mathbb{Q}_{p}$. Let us assume Condition (Ir) for $\mathcal{T}=\mathcal{T}_{\mathcal{F}}^{(i)}$ and the existence of the elements $\tau \in G_{\mathbb{Q}\left(\mu_{p}^{\infty}\right)}$ and $\tau^{\prime} \in G_{\mathbb{Q}}$ which satisfy the following properties. 


\section{T. OCHIAI}

(i) The image of $\tau$ under the representation $G_{\mathbb{Q}} \longrightarrow \operatorname{Aut}(\mathcal{T}) \cong \mathrm{GL}_{2}\left(\mathbb{H}_{\mathcal{F}}^{\text {n.o }}\right)$ has a presentation $\left(\begin{array}{cc}1 & P_{\tau} \\ 0 & 1\end{array}\right)$ under certain choice of basis $\mathcal{T} \cong\left(\mathbb{H}_{\mathcal{F}}^{\text {n.o }}\right)^{\oplus 2}$, where $P_{\tau}$ is a non-zero element of $\mathbb{H}_{\mathcal{F}}^{\text {n.o. }}$.

(ii) The element $\tau^{\prime} \in G_{\mathbb{Q}}$ acts on $\mathcal{T} / \mathfrak{M T}$ via the multiplication by -1 .

Then there exists an integer $m \geqslant 0$ such that we have the following inequality for each height-one prime $\mathfrak{l}$ of $\mathbb{H}_{\mathcal{F}}^{\text {n.o }}$ :

$$
\operatorname{length}_{\mathbb{H}_{\mathcal{F}, \mathfrak{l}}^{\text {n.o }}}\left(\operatorname{Sel}_{\mathcal{T}}\right)_{\mathfrak{l}}^{\vee} \leqslant \operatorname{length}_{\mathbb{H}_{\mathcal{F}, \mathfrak{l}}^{\text {n.o }}}\left(H_{/ f}\left(\mathbb{Q}_{p}, \mathcal{T}^{*}(1)\right) / \operatorname{loc} / f(\mathcal{Z}) \mathbb{H}_{\mathcal{F}}^{\text {n.o }}\right)_{\mathfrak{l}}+\operatorname{ord}_{\mathfrak{l}}\left(P_{\tau}^{m}\right) .
$$

Remark 2.5. In [Och05], we assumed the following condition (ii') in place of the above condition (ii):

(ii') The element $\tau^{\prime} \in G_{\mathbb{Q}}$ acts on $\mathcal{T}$ via the multiplication by -1 .

However, conditions (ii) and (ii') are equivalent to each other by the following lemma.

Lemma 2.6. Let $R$ be a complete Noetherian local ring whose residue field $R / \mathfrak{M}$ is a finite field of characteristic $p>2$ and let $G$ be a subgroup of $\mathrm{GL}_{2}(R)$. We denote by $\bar{G} \subset \mathrm{GL}_{2}(R / \mathfrak{M})$ the image of $G$ under the reduction map $\mathrm{GL}_{2}(R) \longrightarrow \mathrm{GL}_{2}(R / \mathfrak{M})$. Then $G$ contains a scalar matrix of multiplication by -1 if and only if $\bar{G}$ contains the multiplication by -1 .

We omit the proof of this rather elementary lemma, but we remark that condition (ii) is easier to check than condition (ii') (cf. $\S 9$ and Claim 9.11).

Finally, our results combining Theorems 1 and 2 are summarized as follows.

Theorem 3. Let us assume Condition (Ir). Assume further that $\mathbb{H}_{\mathcal{F}}^{\text {n.o }}$ is isomorphic to a two-variable power series algebra $\mathcal{O}\left[\left[X_{1}, X_{2}\right]\right]$. Then the following hold.

(i) The Pontryagin dual $\left(\operatorname{Sel}_{\mathcal{T}}\right)^{\vee}$ of $\operatorname{Sel}_{\mathcal{T}}$ is a finitely generated torsion $\mathbb{H}_{\mathcal{F}}^{\text {n.o }}$-module.

(ii) Suppose that we have elements $\tau \in G_{\mathbb{Q}\left(\mu_{p} \infty\right)}$ and $\tau^{\prime} \in G_{\mathbb{Q}}$ satisfying conditions (i) and (ii) in Theorem 2. Then, there exists an integer $m$ such that we have the following inequality for each height-one prime $\mathfrak{l}$ of $\mathbb{H}_{\mathcal{F}}^{\text {n.o }}$ :

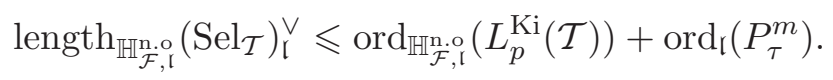

So far, we have given results on the two-variable IMC for nearly ordinary deformations $\mathcal{T}$. The above results are applied to the Iwasawa theory for one-variable specializations $\mathcal{T} / J \mathcal{T}$ for various height-one ideals $J$ of $\mathbb{H}_{\mathcal{F}}^{\text {n.o }}$ as cases (a), (b), (c) and (d) given earlier in this section. In $\S 4.2$, the Selmer group for $\mathcal{T} / J \mathcal{T}$ is studied using Bloch-Kato's method or Greenberg's method and we compare two different definitions. A technique of specialization from two variables to one variable is discussed in $\S 5.1$. Based on these preparations, we discuss the one-variable Iwasawa theory for the deformations (a), (b), (c) and (d) above. For example, by applying Lemma 7.2 to case (a), we have the following corollary to Theorem 3 (see Corollary 7.5).

Corollary 2.7. Assume the same conditions as those in Theorem 2 (and certain technical assumptions given in Corollary 7.5). Then, the following statements are equivalent.

(1) The two-variable IMC holds for $\mathcal{T}$.

(2) The cyclotomic IMC (cf. Conjecture 7.4) formulated by Mazur-Tate-Teitelbaum holds for every specialization $f_{\mathfrak{I}}$ of $\mathcal{F}$ with $\mathfrak{I} \in \mathfrak{X}_{\text {arith }}\left(\mathbb{H}_{\mathcal{F}}^{\text {ord }}\right)_{\geqslant 0}$.

(3) There exists an $\mathfrak{I}_{0} \in \mathfrak{X}_{\text {arith }}\left(\mathbb{H}_{\mathcal{F}}^{\text {ord }}\right)_{\geqslant 0}$ such that the cyclotomic IMC holds for $f_{\mathfrak{I}_{0}}$.

We do not give the proof of this corollary in this section, but it will be given in $\S 7$. Suppose that $\mathcal{F}$ and $\mathcal{T}=\mathcal{T}_{\mathcal{F}}^{(i)}$ satisfy the assumption for the corollary (see Remark 7.6 for some of the explicit sufficient conditions for $\mathcal{T}$ to satisfy the condition). By applying the implication (3) $\Longrightarrow(2)$ of 


\section{ON THE TWO-VARIABLE IWASAWA MAIN CONJECTURE}

Corollary 2.7, we show that Conjecture 7.4 holds for every specialization $f_{\mathfrak{I}}$ of $\mathcal{F}$ with $w(\mathfrak{I}) \geqslant 0$ once the cyclotomic IMC holds for a certain specialization $f_{\mathfrak{I}_{0}}$ in $\mathcal{F}$ with $w\left(\mathfrak{I}_{0}\right) \geqslant 0$. Thus, we have an infinite family of modular forms $\left\{f_{\mathfrak{I}}\right\}_{\mathfrak{I} \in \mathfrak{X}_{\text {arith }}\left(\mathbb{H}_{\mathcal{F}} \text { ord }\right)_{\geqslant 0}}$ where the cyclotomic IMC holds for every member of the family. A recent paper [EPW06] also proves a similar result that the cyclotomic IMC

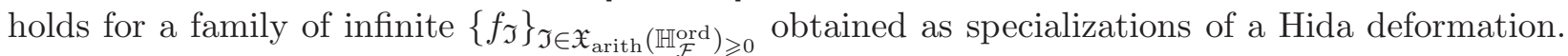
Their method of the proof is not related to the two-variable IMC as in this paper, but they apply a clever use of the congruence developed by Greenberg and Vatsal [GV00]. For this reason, they assume that the vanishing of the cyclotomic Iwasawa $\mu$-invariant $\mu\left(f_{\mathfrak{I}_{0}}\right)$ is zero for a certain specialization $f_{\mathfrak{I}_{0}}$. The advantage of our result in Corollary 2.7 is that we do not have to assume the condition $\mu\left(f_{\mathfrak{I}_{0}}\right)=0$.

Another thing we should remark on is that Skinner and Urban recently announced the cyclotomic IMC for ordinary eigen-cuspforms $f$ with certain technical conditions, assuming the conjecture on the existence of Galois representations for modular forms on $\mathrm{U}(2,2)$. By combining their result with the implication $(3) \Longrightarrow(1)$ of Corollary 2.7, we prove the two-variable IMC (cf. Conjecture 2.4) under certain conditions.

As far as we know, the one-variable IMC in cases (b), (c) and (d) is not known and has not been formulated previously. Based on the preparation in $\S \S 4,5$ and 6 , we formulate these conjectures in $\S 7$. We refer the reader to $\S 7$ for the formulation of the conjectures and our results.

Since only a few things are known about the two-variable Iwasawa theory, we would like explicit examples which help us to develop our future perspective. As an attempt, we study the case of Ramanujan's cuspform $\Delta=q \prod_{n \geqslant 1}\left(1-q^{n}\right)^{24} \in S_{12}\left(\mathrm{SL}_{2}(\mathbb{Z})\right)$. For each prime number $p$ such that $p \nmid a_{p}(\Delta)$, we have a unique $\Lambda$-adic newform $\mathcal{F}(\Delta)$ which contains $\Delta$ at weight 12 . For each integer $i$ with $0 \leqslant i \leqslant p-2$, we have a nearly ordinary deformation $\mathcal{T}_{\mathcal{F}(\Delta)}^{(i)}$.

Proposition 2.8. Let $p \geqslant 11$ be a prime number with $p \nmid a_{p}(\Delta)$. Assume that $1 \leqslant i \leqslant 11$ and $p \leqslant 10000$. Let $\mathcal{T}$ be $\mathcal{T}_{\mathcal{F}(\Delta)}^{(i)}$.

(1) Except for $(p, i)=(11,1),(23,1)$ and $(691,1)$, we have $\operatorname{Sel}_{\mathcal{T}}=0$ and $L_{p}^{\mathrm{Ki}}(\mathcal{T})$ is a unit.

(2) When $p=11$ and $i=1, \operatorname{Sel}_{\mathcal{T}}^{\vee}$ is isomorphic to $\mathbb{Z}_{p}\left[\left[\Gamma \times \Gamma^{\prime}\right]\right] /\left(\gamma^{2}-\kappa^{2}\left(\gamma^{\prime}\right) \gamma^{\prime}\right)$ and we have the equality of ideal $\left(\gamma^{2}-\kappa^{2}\left(\gamma^{\prime}\right) \gamma^{\prime}\right)=\left(L_{p}^{\mathrm{Ki}}(\mathcal{T})\right)$.

Remark 2.9. Thus, in particular, the two-variable IMC of $\Delta$ holds for all $p \leqslant 10000$ and $0 \leqslant i \leqslant 10$ except for $(p, i)=(23,1)$ and $(p, i)=(691,1)$. For $(p, i)=(23,1)$, it is easy to see that $L_{p}^{\mathrm{Ki}}(\mathcal{T})$ is not a unit by the interpolation property in Theorem 6.7 since $a_{p}(\Delta)-1 \equiv 0$ modulo 23 . The image of modulo 23 representation for $\Delta$ is dihedral and thus condition (ii) in Theorem 2 is not satisfied. It is our future project to generalize the results in [Och05] so that Theorem 2 is true in the case $p=23$. For $p=691$, the residual representation is no longer irreducible (Condition (Ir) is not satisfied) and the choice of lattice $\mathcal{T}$ is not unique for a given $\mathcal{F}$. We will treat the IMC for residually reducible deformations in a forthcoming paper.

\section{Local monodromy on $\mathbb{T}_{\mathcal{F}}^{\text {ord }}$}

For later use in $\S \S 4,5$ and 7 , we study the action $\rho_{\mathcal{F}}$ of the inertia group $I_{v}$ at primes $v \mid N$ acting on the Hida deformation $\mathbb{T}_{\mathcal{F}}^{\text {ord }}$ associated to a $\Lambda$-adic newform $\mathcal{F}$ of tame conductor $N$ introduced in $\S 2$. We will keep the notation of the previous section. Throughout the paper, we denote by $\Sigma$ the set of primes of $\mathbb{Q}$ which consists of finite primes dividing $N p$ and the infinite prime $\{\infty\}$. The result of this section is summarized in Theorem 3.3. The reader who is mainly interested in the Selmer group or in the $p$-adic $L$-function can skip this section by admitting Theorem 3.3.

We prepare the following lemma. 


\section{T. OCHIAI}

Lemma 3.1. Let $G \subset \mathrm{Aut}_{\mathbb{H}_{\mathcal{F}}^{\text {ord }}}\left(\mathbb{T}_{\mathcal{F}}^{\text {ord }}\right)$ be a finite subgroup. For each $\mathfrak{I} \in \mathfrak{X}_{\text {arith }}\left(\mathbb{H}_{\mathcal{F}}^{\text {ord }}\right)_{\geqslant 0}$, $G$ is mapped into $\operatorname{Aut}_{\mathcal{O}_{\mathfrak{I}}}\left(T_{f_{\mathfrak{I}}}\right)$ under the specialization $\operatorname{Aut}_{\mathbb{H}_{\mathcal{F}} \text { ord }}\left(\mathbb{T}_{\mathcal{F}}^{\text {ord }}\right) \rightarrow \operatorname{Aut}_{\mathcal{O}_{\mathfrak{I}}}\left(T_{f_{\mathfrak{I}}}\right)$.

Proof. By fixing a basis of $\mathbb{T}_{\mathcal{F}}^{\text {ord }}$, we have isomorphisms $\mathbb{T}_{\mathcal{F}}^{\text {ord }} \cong\left(\mathbb{H}_{\mathcal{F}}^{\text {ord }}\right)^{\oplus 2}$ and $T_{f_{\mathfrak{I}}} \cong\left(\mathcal{O}_{\mathfrak{I}}\right)^{\oplus 2}$. Suppose that there exists an element $g \in G$ which is mapped to a trivial element on $\operatorname{Aut}_{\mathcal{O}_{\mathfrak{I}}}\left(T_{f_{\mathfrak{I}}}\right) \cong \mathrm{GL}_{2}\left(\mathcal{O}_{\mathfrak{I}}\right)$. Since the order of $g$ is finite, by extending the coefficients of $\mathbb{H}_{\mathcal{F}}^{\text {ord }}$ if necessary, we may assume that $g$ is conjugate to a diagonal matrix $\left(\begin{array}{cc}u & 0 \\ 0 & u^{\prime}\end{array}\right) \in \mathrm{GL}_{2}\left(\mathbb{H}_{\mathcal{F}}^{\text {ord }}\right)$ with $u$ and $u^{\prime}$ roots of unity. This completes the proof since the roots of unity in $\left(\mathbb{H}_{\mathcal{F}}^{\text {ord }}\right)^{\times}$are disjoint from $\operatorname{Ker}\left[\left(\mathbb{H}_{\mathcal{F}}^{\text {ord }}\right)^{\times} \rightarrow\left(\mathcal{O}_{\mathfrak{I}}\right)^{\times}\right]$.

Since $v \in \Sigma \backslash\{p, \infty\}$, the action of $I_{v}$ on $\mathbb{T}_{\mathcal{F}}^{\text {ord }}$ is non-trivial. By the above lemma, we consider the following case.

(A) The image $\rho_{\mathcal{F}}\left(I_{v}\right)$ in $\operatorname{Aut}_{\mathbb{H}_{\mathcal{F}}^{\text {ord }}}\left(\mathbb{T}_{\mathcal{F}}^{\text {ord }}\right)$ is a finite subgroup.

In this case, the action of $I_{v}$ on $\left(\mathbb{T}_{\mathcal{F}}^{\text {ord }}\right)^{*}=\operatorname{Hom}_{\mathbb{H}_{\mathcal{F}} \text { ord }}\left(\mathbb{T}_{\mathcal{F}}^{\text {ord }}, \mathbb{H}_{\mathcal{F}}^{\text {ord }}\right)$ also factors through a finite quotient of $I_{v}$. Hence there exist a finite flat extension $\mathcal{O}$ of $\mathbb{Z}_{p}$ contained in $\mathbb{H}_{\mathcal{F}}^{\text {ord }}$ and a finite type $\mathcal{O}$-module $M$ with $\operatorname{rank}_{\operatorname{Frac}(\mathcal{O})}\left(M \otimes_{\mathcal{O}} \operatorname{Frac}(\mathcal{O})\right) \leqslant 1$ such that the coinvariant quotient $\left(\left(\mathbb{T}_{\mathcal{F}}^{\text {ord }}\right)^{*}\right)_{I_{v}}$ is isomorphic to $M \otimes_{\mathcal{O}} \mathbb{H}_{\mathcal{F}}^{\text {ord }}$.

Next, we discuss the following case.

(B) The image $\rho_{\mathcal{F}}\left(I_{v}\right)$ in $\operatorname{Aut}_{\mathbb{H}_{\mathcal{F}} \text { ord }}\left(\mathbb{T}_{\mathcal{F}}^{\text {ord }}\right)$ is an infinite subgroup.

In this case, it is not difficult to see that there exists an arithmetic point $\mathfrak{I} \in \mathfrak{X}_{\text {arith }}\left(\mathbb{H}_{\mathcal{F}}^{\text {ord }}\right)_{\geqslant 0}$ such that the action of $I_{v}$ on $T_{f_{\mathfrak{I}}} \cong \mathbb{T}_{\mathcal{F}}^{\text {ord }} / \operatorname{Ker}(\mathfrak{I}) \mathbb{T}_{\mathcal{F}}^{\text {ord }}$ does not factor through a finite quotient of $I_{v}$. Let us fix one such $\mathfrak{I}_{0} \in \mathfrak{X}_{\text {arith }}\left(\mathbb{H}_{\mathcal{F}}^{\text {ord }}\right)_{\geqslant 0}$ for a while. We note that the action of $I_{v}$ on $T_{f_{\mathfrak{I}_{0}}}$ can be infinite only when the local automorphic representation $\pi_{v}\left(\mathfrak{I}_{0}\right)$ of $\mathrm{GL}_{2}\left(\mathbb{Q}_{v}\right)$ associated to $f_{\mathfrak{I}_{0}}$ is a special representation. Hence the local Galois representation $G_{\mathbb{Q}_{v}} \longrightarrow \mathrm{GL}_{2}\left(\mathcal{O}_{\mathfrak{I}}\right)$ for $f_{\mathfrak{I}_{0}}$ is represented by a matrix $\left(\begin{array}{ll}\chi & * \\ 0 & \chi^{\prime}\end{array}\right)$ such that $\left.\chi\right|_{I_{v}}=\left.\chi^{\prime}\right|_{I_{v}}$ and $\chi^{\prime} \chi^{-1}=||^{ \pm}$, where || is the absolute value character $G_{\mathbb{Q}_{v}} \longrightarrow G_{\mathbb{Q}_{v}}^{\text {ab }} \stackrel{\sim}{\longrightarrow} \mathbb{Q}_{v}^{\times} \longrightarrow||_{v}$. Since a finite-order character of $G_{\mathbb{Q}_{v}}$ is always the localization of a finite-order character of $G_{\mathbb{Q}}$, we have a Dirichlet character $\eta_{0}$ with $v$-primary conductor such that the action of $I_{v}$ on $T_{f_{\mathfrak{I}_{0}}} \otimes \eta_{0}=T_{f_{\mathfrak{I}_{0} \otimes \eta_{0}}}$ is unipotent. Let us now recall the structure on the inertia group $I_{v}$. The group $I_{v}$ has the filtration $P \subset Q \subset I_{v}$ such that $P$ is the maximal pro- $v$ subgroup of $I_{v}$ and $I_{v} / Q$ is isomorphic to $\mathbb{Z}_{p}$. Since $Q / P$ is isomorphic to $\prod_{l \neq v, p} \mathbb{Z}_{l}, Q$ has no non-trivial $p$-primary subquotient. This immediately implies the following lemma.

LEMma 3.2. Let $v \in \Sigma \backslash\{p, \infty\}$.

(1) The image $\rho_{\mathcal{F}}(Q)$ is a finite subgroup of $\operatorname{Aut}_{\mathbb{H}_{\mathcal{F}}}\left(\mathbb{T}_{\mathcal{F}}^{\text {ord }}\right)$.

(2) For each $\mathfrak{I} \in \mathfrak{X}_{\text {arith }}\left(\mathbb{H}_{\mathcal{F}}^{\text {ord }}\right)_{\geqslant 0}$, the group $\rho_{\mathcal{F}}(Q)$ is mapped into $\operatorname{Aut}_{\mathcal{O}_{\mathfrak{I}}}\left(T_{f_{\mathfrak{I}}}\right)$ under the specialization $\operatorname{Aut}_{\mathbb{H}_{\mathcal{F}}^{\text {ord }}}\left(\mathbb{T}_{\mathcal{F}}^{\text {ord }}\right) \rightarrow \operatorname{Aut}_{\mathcal{O}_{\mathfrak{I}}}\left(T_{f_{\mathfrak{I}}}\right)$.

Proof. For the proof, we note that the prime-to- $p$ part of $\mathrm{Aut}_{\mathbb{H}_{\mathcal{F}} \text { ord }}\left(\mathbb{T}_{\mathcal{F}}^{\text {ord }}\right)$ is finite and that the kernel of $\operatorname{Aut}_{\mathbb{H}_{\mathcal{F}}^{\text {ord }}}\left(\mathbb{T}_{\mathcal{F}}^{\text {ord }}\right) \rightarrow \operatorname{Aut}_{\mathcal{O}_{\mathfrak{I}}}\left(T_{f_{\mathfrak{I}}}\right)$ is a pro- $p$ group.

Since the action of $I_{v}$ on $T_{f_{\mathfrak{I}} \otimes \eta_{0}}$ is unipotent, the subgroup $Q$ acts trivially on $T_{f_{\mathfrak{I}} \otimes \eta_{0}}$. By Lemma 3.2, $Q$ acts trivially on $\mathbb{T}_{\mathcal{F}}^{\text {ord }} \otimes \eta_{0}=\mathbb{T}_{\mathcal{F} \otimes \eta_{0}}^{\text {ord }}$, where $\mathcal{F} \otimes \eta_{0}$ is the $\Lambda$-adic newform obtained as the twist of $\mathcal{F}$ by $\eta_{0}$. Let $\gamma$ be a topological generator of $I_{v} / Q \cong \mathbb{Z}_{p}$. By assumption, the action of $\gamma$ on $T_{f_{\mathfrak{I}_{0}} \otimes \eta_{0}}$ is represented by a non-trivial unipotent matrix. Let $\left(\mathbb{T}_{\mathcal{F}}^{\text {ord }} \otimes \eta_{0}\right)^{\text {ss }}$ be the semi-simplification as an $I_{v}$-module. Then, the action of $\gamma$ on $\left(\mathbb{T}_{\mathcal{F} \otimes \eta_{0}}^{\text {ord }}\right)^{\mathrm{ss}}=\left(\mathbb{T}_{\mathcal{F}}^{\text {ord }} \otimes \eta_{0}\right)^{\text {ss }}$ is represented by a matrix $\left(\begin{array}{cc}a & 0 \\ 0 & a^{\prime}\end{array}\right)$ with $a, a^{\prime} \in\left(\mathbb{H}_{\mathcal{F}}^{\text {ord }}\right)^{\times}$. If $a$ or $a^{\prime}$ is not a root of unity, there exists $\mathfrak{I} \in \mathfrak{X}_{\text {arith }}\left(\mathbb{H}_{\mathcal{F}}^{\text {ord }}\right)_{\geqslant 0}$ such that the action of $I_{v}$ on $\left(\mathbb{T}_{\mathcal{F} \otimes \eta_{0}}^{\text {ord }}\right)^{\text {ss }} / \operatorname{Ker}(\mathfrak{I})\left(\mathbb{T}_{\mathcal{F} \otimes \eta_{0}}^{\text {ord }}\right)^{\text {ss }}=\left(T_{f_{\mathfrak{I}}}\right)^{\text {ss }}$ is of infinite order. It is impossible for a 


\section{ON THE TWO-VARIABLE IWASAWA MAIN CONJECTURE}

representation of $I_{v}$ associated to a cuspform. Hence, $a$ and $a^{\prime}$ are roots of unity. Since $a$ and $a^{\prime}$ are congruent to 1 modulo $\operatorname{Ker}\left(\mathfrak{I}_{0}\right)$, we show that $a=a^{\prime}=1$ by a similar discussion as in Lemma 3.1 for $\left(\mathbb{T}_{\mathcal{F} \otimes \eta_{0}}^{\text {ord }}\right)^{\text {ss }}$. Thus, the action of $\gamma$ on $\mathbb{T}_{\mathcal{F} \otimes \eta_{0}}^{\text {ord }}$ is represented by a unipotent matrix $\left(\begin{array}{ll}1 & b \\ 0 & 1\end{array}\right) \in \mathrm{GL}_{2}\left(\mathbb{H}_{\mathcal{F}}^{\text {ord }}\right)$. Recall that the $v$-order of the tame conductor of $f_{\mathfrak{I} \otimes \eta_{0}}$ is constant when $\mathfrak{I}$ varies in $\mathfrak{X}_{\text {arith }}\left(\mathbb{H}_{\mathcal{F}}^{\text {ord }}\right)_{\geqslant 0}$ by applying [Hid86a, Corollary 3.7] to $\mathcal{F} \otimes \eta_{0}$. Thus, $\mathfrak{I}(b)$ are not zero for every $\mathfrak{I} \in \mathfrak{X}_{\text {arith }}\left(\mathbb{H}_{\mathcal{F}}^{\text {ord }}\right) \geqslant 0$. We conclude that $\left(\left(\mathbb{T}_{\mathcal{F}}^{\text {ord }}\right)^{*}\right)_{I_{v}}$ is isomorphic to $\mathbb{H}_{\mathcal{F}}^{\text {ord }} /(1-u) \oplus \mathbb{H}_{\mathcal{F}}^{\text {ord }} /(b, 1-u)$, where $u$ is a root of unity which generates the group of the values of $\eta_{0}$.

Summarizing the above argument, we have the following theorem.

Theorem 3.3. Let $v \in \Sigma \backslash\{p, \infty\}$.

(1) If the image of $I_{v}$ on $\mathrm{Aut}_{\mathbb{H}_{\mathcal{F}} \text { ord }}\left(\mathbb{T}_{\mathcal{F}}^{\text {ord }}\right)$ is finite, there exist a finite flat extension $\mathcal{O}$ of $\mathbb{Z}_{p}$ contained in $\mathbb{H}_{\mathcal{F}}^{\text {ord }}$ and a finite type $\mathcal{O}$-module $M$ with $\operatorname{rank}_{\operatorname{Frac}(\mathcal{O})}\left(M \otimes_{\mathcal{O}} \operatorname{Frac}(\mathcal{O})\right) \leqslant 1$ such that the coinvariant quotient $\left(\left(\mathbb{T}_{\mathcal{F}}^{\text {ord }}\right)^{*}\right)_{I_{v}}$ is isomorphic to $M \otimes \mathcal{O} \mathbb{H}_{\mathcal{F}}^{\text {ord }}$.

(2) If the image of $I_{v}$ on $\mathrm{Aut}_{\mathbb{H}_{\mathcal{F}} \text { ord }}\left(\mathbb{T}_{\mathcal{F}}^{\text {ord }}\right)$ is infinite, $\left(\left(\mathbb{T}_{\mathcal{F}}^{\text {ord }}\right)^{*}\right)_{I_{v}}$ is isomorphic to $\mathbb{H}_{\mathcal{F}}^{\text {ord }} /(1-u) \oplus$ $\mathbb{H}_{\mathcal{F}}^{\text {ord }} /(b, 1-u)$ where $b$ is an element in $\mathbb{H}_{\mathcal{F}}^{\text {ord }}$ such that $\mathfrak{I}(b) \neq 0$ for every $\mathfrak{I} \in \mathfrak{X}_{\text {arith }}\left(\mathbb{H}_{\mathcal{F}}^{\text {ord }}\right)_{\geqslant 0}$ and $u$ is a certain root of unity in $\left(\mathbb{H}_{\mathcal{F}}^{\text {ord }}\right)^{\times}(u=1$ is possible $)$.

The following remark explains Theorem 3.3 from the theory of admissible representations and the local Langlands correspondence for $\mathrm{GL}_{2}$.

Remark 3.4. In case (A) of this section, the admissible representation $\pi_{v}(\mathfrak{I})$ of $\mathrm{GL}_{2}\left(\mathbb{Q}_{p}\right)$ corresponding to $f_{\mathfrak{I}}$ is a supercuspidal representation or a principal series at each $\mathfrak{I} \in \mathfrak{X}_{\text {arith }}\left(\mathbb{H}_{\mathcal{F}}^{\text {ord }}\right)_{\geqslant 0}$. Further, if $\pi_{v}(\mathfrak{I})$ is a supercuspidal representation (respectively a principal series) at one of $\mathfrak{I} \in \mathfrak{X}_{\text {arith }}\left(\mathbb{H}_{\mathcal{F}}^{\text {ord }}\right)_{\geqslant 0}$, $\pi_{v}(\mathfrak{I})$ are supercuspidal representations (respectively a principal series) at every $\mathfrak{I} \in \mathfrak{X}_{\text {arith }}\left(\mathbb{H}_{\mathcal{F}}^{\text {ord }}\right) \geqslant 0$. In case $(\mathrm{B}), \pi_{v}(\mathfrak{I})$ is a special representation at each $\mathfrak{I} \in \mathfrak{X}_{\text {arith }}\left(\mathbb{H}_{\mathcal{F}}^{\text {ord }}\right)_{\geqslant 0}$.

\section{Selmer groups for Galois deformations}

In this section, we review the definition of Selmer groups for a two-variable nearly ordinary deformation $\mathcal{T}=\mathcal{T}_{\mathcal{F}}^{(i)}$ and for its various specializations $\mathcal{T} / \mathfrak{A T}$ by ideals $\mathfrak{A} \subset \mathbb{H}_{\mathcal{F}}^{\text {n.o. }}$. We also give some fundamental properties on these Selmer groups.

Let $\mathcal{A}$ be the discrete Galois representation $\mathcal{T} \otimes_{\mathbb{H}_{\mathcal{F}}^{\text {n.o }}} \operatorname{Hom}_{\mathbb{Z}_{p}}\left(\mathbb{H}_{\mathcal{F}}^{\text {n.o }}, \mathbb{Q}_{p} / \mathbb{Z}_{p}\right)$. We denote by $\mathbb{Q}_{\Sigma}$ the maximal Galois extension of $\mathbb{Q}$ which is unramified outside $\Sigma$.

\subsection{Selmer groups over discrete valuation rings}

Let $(j, k)$ be a pair of integers satisfying $1 \leqslant j \leqslant k-1$ and let $\Delta_{s, t}^{(j, k)}=\left(\gamma^{p^{s}}-\chi^{j}\left(\gamma^{p^{s}}\right), \gamma^{\prime p^{t}}-\kappa^{k-2}\left(\gamma^{\prime p^{t}}\right)\right)$ be a height-two ideal of $\mathbb{H}_{\mathcal{F}}^{\text {n.o }}$. We denote by $A_{s, t}^{(j, k)}$ the $\Delta_{s, t}^{(j, k)}$-torsion part $\mathcal{A}\left[\Delta_{s, t}^{(j, k)}\right]$ of $\mathcal{A}$, which is identified with $\left(\mathcal{T} / \Delta_{s, t}^{(j, k)} \mathcal{T}\right) \otimes_{\mathbb{Z}_{p}} \mathbb{Q}_{p} / \mathbb{Z}_{p}$. Note the following.

(1) $\mathcal{T} / \Delta_{s, t}^{(j, k)} \mathcal{T}$ is free of finite rank over $\mathbb{Z}_{p}$

(2) The $p$-adic representation $\left(\mathcal{T} / \Delta_{s, t}^{(j, k)} \mathcal{T}\right) \otimes_{\mathbb{Z}_{p}} \mathbb{Q}_{p}$ is isomorphic to $\bigoplus_{f}\left(V_{f} \otimes \chi^{j} \omega^{i}\right) \otimes_{\mathbb{Z}_{p}} \mathbb{Z}_{p}\left[\Gamma / \Gamma^{p^{s}}\right](\widetilde{\chi})$, where $f$ runs ordinary eigen-cuspforms of weight $k$ for $\Gamma_{1}\left(N p^{t}\right)$ such that the residual representation for $f$ are isomorphic to that of $\mathcal{T} \otimes \omega^{-i}$. Here, $\mathbb{Z}_{p}\left[\Gamma / \Gamma^{p^{s}}\right](\widetilde{\chi})$ is a free $\mathbb{Z}_{p}\left[\Gamma / \Gamma^{p^{s}}\right]$-module of rank one on which $G_{\mathbb{Q}}$ acts via the tautological character $\tilde{\chi}: G_{\mathbb{Q}} \rightarrow \Gamma / \Gamma^{p^{s}} \hookrightarrow \mathbb{Z}_{p}\left[\Gamma / \Gamma^{p^{s}}\right]^{\times}$. We recall that $i$ is a fixed integer which is implicitly contained in the definition of $\mathcal{T}=\mathcal{T}_{\mathcal{F}}^{(i)}$. 


\section{T. OCHIAI}

For any $\operatorname{Gal}\left(\mathbb{Q}_{\Sigma} / \mathbb{Q}\right)$-module $T$ which is free of finite rank over $\mathbb{Z}_{p}$, Selmer groups are defined as a subgroup of $H^{1}\left(\mathbb{Q}_{\Sigma} / \mathbb{Q}, A\right)$, where $A=T \otimes_{\mathbb{Z}_{p}} \mathbb{Q}_{p} / \mathbb{Z}_{p}$. Once we fix a local condition $H_{?}^{1}\left(\mathbb{Q}_{v}, A\right) \subset$ $H^{1}\left(\mathbb{Q}_{v}, A\right)$ at each $v \in \Sigma \backslash\{\infty\}$, we define a Selmer group $\mathrm{Sel}_{T}^{?}$ as follows (? is BK (Bloch-Kato) or $\operatorname{Gr}($ Greenberg)):

$$
\operatorname{Sel}_{T}^{?}=\operatorname{Ker}\left[H^{1}\left(\mathbb{Q}_{\Sigma} / \mathbb{Q}, A\right) \longrightarrow \prod_{v \in \Sigma} \frac{H^{1}\left(\mathbb{Q}_{v}, A\right)}{H_{?}^{1}\left(\mathbb{Q}_{v}, A\right)}\right] .
$$

For $v \in \Sigma \backslash\{p, \infty\}$, one of the local conditions is given by the unramified part

$$
H_{\mathrm{ur}}^{1}\left(\mathbb{Q}_{v}, A\right)=\operatorname{Ker}\left[H^{1}\left(\mathbb{Q}_{v}, A\right) \longrightarrow H^{1}\left(I_{v}, A\right)\right],
$$

where $I_{v}$ is the inertia subgroup at $v$. Let $V=T \otimes_{\mathbb{Z}_{p}} \mathbb{Q}_{p}$. We define 'the finite part':

$$
H_{f}^{1}\left(\mathbb{Q}_{v}, A\right)=\operatorname{pr}\left(H_{\mathrm{ur}}^{1}\left(\mathbb{Q}_{v}, V\right)\right),
$$

where pr: $H^{1}\left(\mathbb{Q}_{v}, V\right) \longrightarrow H^{1}\left(\mathbb{Q}_{v}, A\right)$ is the map induced by the projection map $V \rightarrow A=V / T$ of $G_{\mathbb{Q}_{v}}$-modules and $H_{\mathrm{ur}}^{1}\left(\mathbb{Q}_{v}, V\right)=\operatorname{Ker}\left[H^{1}\left(\mathbb{Q}_{v}, V\right) \longrightarrow H^{1}\left(I_{v}, V\right)\right]$.

We also give local conditions at $p$.

(1) Greenberg's local condition $H_{\mathrm{Gr}}^{1}\left(\mathbb{Q}_{p}, A\right) \subset H^{1}\left(\mathbb{Q}_{p}, A\right)$ is defined as

$$
H_{\mathrm{Gr}}^{1}\left(\mathbb{Q}_{p}, A\right)=\operatorname{Ker}\left[H^{1}\left(\mathbb{Q}_{p}, A\right) \longrightarrow H^{1}\left(I_{p}, \mathrm{~F}^{-} A\right)\right],
$$

where $\mathrm{F}^{-} A$ is a $G_{\mathbb{Q}_{p}}$-module which is defined to be the quotient $A / \mathrm{F}^{+} A$.

(2) Bloch-Kato defined $H_{f}^{1}\left(\mathbb{Q}_{p}, A\right)$, called 'the finite part', as $H_{f}^{1}\left(\mathbb{Q}_{p}, A\right)=\operatorname{pr}\left(H_{f}^{1}\left(\mathbb{Q}_{p}, V\right)\right)$, where

$$
H_{f}^{1}\left(\mathbb{Q}_{p}, V\right)=\operatorname{Ker}\left[H^{1}\left(\mathbb{Q}_{p}, V\right) \longrightarrow H^{1}\left(\mathbb{Q}_{p}, V \otimes B_{\text {crys }}\right)\right],
$$

by using the ring of $p$-adic periods $B_{\text {crys }}$ defined by Fontaine (cf. [Fon94]).

Selmer groups $\mathrm{Sel}_{T}^{\mathrm{BK}}$ and $\mathrm{Sel}_{T}^{\mathrm{Gr}}$ according to [BK90] and [Gre87] are defined by the following condition (cf. (3)).

\begin{tabular}{ccc}
\hline & $\operatorname{Sel}_{T}^{\mathrm{BK}}$ & $\operatorname{Sel}_{T}^{\mathrm{Gr}}$ \\
\hline$H_{?}^{1}\left(\mathbb{Q}_{v}, A\right)$ for $v \in \Sigma \backslash\{p, \infty\}$ & $H_{f}^{1}$ & $H_{\mathrm{ur}}^{1}$ \\
$H_{?}^{1}\left(\mathbb{Q}_{p}, A\right)$ & $H_{f}^{1}\left(\mathbb{Q}_{p}, A\right)$ & $H_{\mathrm{Gr}}^{1}\left(\mathbb{Q}_{p}, A\right)$ \\
\hline
\end{tabular}

Recall that we have the following proposition (cf. [Och03, §4]).

Proposition 4.1. Let us assume that $1 \leqslant j \leqslant k-1$. Then $H_{f}^{1}\left(\mathbb{Q}_{p}, A_{s, t}^{(j, k)}\right)$ is the maximal divisible subgroup of $H_{\mathrm{Gr}}^{1}\left(\mathbb{Q}_{p}, A_{s, t}^{(j, k)}\right)$ for each pair of integers $(s, t) \geqslant(0,0)$.

We have the following corollary of Proposition 4.1.

Corollary 4.2. Let us assume that $1 \leqslant j \leqslant k-1$. We denote by $T_{s, t}^{(j, k)}$ the representation $\mathcal{T} / \Delta_{s, t}^{(j, k)} \mathcal{T}$, which is free of finite rank over $\mathbb{Z}_{p}$ for each $(s, t) \geqslant(0,0)$. Then $\operatorname{Sel}_{T_{s, t}^{(j, k)}}^{\mathrm{BK}}$ is a subgroup of $\mathrm{Sel}_{T_{s, t}^{(j, k)}}^{\mathrm{Gr}}$ with finite index.

Remark 4.3. Let $T$ be a $G_{\mathbb{Q}^{-}}$-module which is a quotient $\mathcal{T} / J \mathcal{T}$ by a height-two ideal (not necessarily a prime ideal) $J \subset \mathbb{H}_{\mathcal{F}}^{\text {n.o }}$. Assume that there is a pair $(j, k)$ with $1 \leqslant j \leqslant k-1$ such that $T$ is dominated by $T_{s, t}^{(j, k)}$ for sufficiently large $s, t$. Since $T$ is free of finite rank over $\mathbb{Z}_{p}$, we define $\mathrm{Sel}_{T}^{\mathrm{BK}}$ as in the previous subsection. We also define $\mathrm{Sel}_{T}^{\mathrm{Gr}}$ by means of the $G_{\mathbb{Q}_{p}}$-stable filtration $\mathrm{F}^{+} T$ induced from $\mathrm{F}^{+} \mathcal{T}$. Then, the same results as Proposition 4.1 and Corollary 4.2 hold. 


\section{ON THE TWO-VARIABLE IWASAWA MAIN CONJECTURE}

\subsection{Selmer groups over one-variable Iwasawa algebras}

In this subsection, we give Selmer groups for specializations $\mathcal{T}_{J}=\mathcal{T} / J \mathcal{T}$ at height-one primes $J$ of $\mathbb{H}_{\mathcal{F}}^{\text {n.o }}$ in cases (a), (b), (c) and (d) in $\S 2$. Recall that $J$ is given as follows in each case:

(a) $J$ is $I=\operatorname{Ker}(\mathfrak{I}) \mathbb{H}_{\mathcal{F}}^{\text {n.o }}$ for $\mathfrak{I} \in \mathfrak{X}_{\text {arith }}\left(\mathbb{H}_{\mathcal{F}}^{\text {ord }}\right)_{\geqslant 0}$;

(b) $J$ is $(\gamma-\chi(\gamma)) \subset \mathbb{H}_{\mathcal{F}}^{\text {n.o }}$;

(c) $J$ is $\left(\gamma-\kappa\left(\gamma^{\prime}\right) \gamma^{\prime}\right) \subset \mathbb{H}_{\mathcal{F}}^{\text {n.o }}$;

(d) $J$ is $\left(\gamma^{2}-\kappa^{2}\left(\gamma^{\prime}\right) \gamma^{\prime}\right) \subset \mathbb{H}_{\mathcal{F}}^{\text {n.o }}$.

In case $(\mathrm{a}), \mathbb{H}_{\mathcal{F}}^{\text {n.o }} / J \mathbb{H}_{\mathcal{F}}^{\text {n.o }}$ is isomorphic to $\mathcal{O}_{\mathfrak{J}}[[\Gamma]]$. In cases $(\mathrm{b}),(\mathrm{c})$ and $(\mathrm{d}), \mathbb{H}_{\mathcal{F}}^{\text {n.o }} / J \mathbb{H}_{\mathcal{F}}^{\text {n.o }}$ is isomorphic to $\mathbb{H}_{\mathcal{F}}^{\text {ord }}$. The Greenberg-type Selmer group $\operatorname{Sel}_{J}^{\mathrm{Gr}}$ for $\mathcal{T}_{J}$ is defined by

$$
\operatorname{Sel}_{J}^{\mathrm{Gr}}=\operatorname{Ker}\left[H^{1}\left(\mathbb{Q}_{\Sigma} / \mathbb{Q}, \mathcal{A}[J]\right) \longrightarrow \frac{H^{1}\left(\mathbb{Q}_{p}, \mathrm{~F}^{-} \mathcal{A}[J]\right)}{H_{\mathrm{Gr}}^{1}\left(\mathbb{Q}_{p}, \mathrm{~F}^{-} \mathcal{A}[J]\right)} \oplus \underset{v \in \Sigma \backslash\{p, \infty\}}{\bigoplus} \frac{H^{1}\left(\mathbb{Q}_{v}, \mathcal{A}[J]\right)}{H_{\mathrm{ur}}^{1}\left(\mathbb{Q}_{v}, \mathcal{A}[J]\right)}\right] .
$$

In each of the above four cases, let us take a system of height-one ideals (not necessarily prime ideals) $\left\{H_{u} \subset \mathbb{H}_{\mathcal{F}}^{\text {n.o }}\right\}_{u \geqslant 1}$ of $\mathbb{H}_{\mathcal{F}}^{\text {n.o }}$ with the following properties.

(1) We have $H_{u} \supset H_{u+1}$ for each $u \geqslant 1$ and $\bigcap_{u \geqslant 1} H_{u}=0$.

(2) $\mathbb{H}_{\mathcal{F}}^{\text {n.o }} /\left(J, H_{u}\right)$ is finite flat over $\mathbb{Z}_{p}$ for each $u \geqslant 1$.

For each $u \geqslant 1, \operatorname{Sel}_{\mathcal{T} /\left(J, H_{u}\right) \mathcal{T}}^{\mathrm{Gr}}$ is defined as in $\S 4.1$ by using the filtration $\mathrm{F}^{ \pm}\left(\mathcal{T} /\left(J, H_{u}\right) \mathcal{T}\right):=$ $\mathrm{F}^{ \pm} \mathcal{T} /\left(J, H_{u}\right) \mathrm{F}^{ \pm} \mathcal{T}$. Further, $\operatorname{Sel}_{J}^{\mathrm{Gr}}$ is isomorphic to $\lim _{u \geqslant 1} \operatorname{Sel}_{\mathcal{T} /\left(J, H_{u}\right) \mathcal{T}}^{\mathrm{Gr}}$ by definition. On the other hand, the Bloch-Kato-type Selmer group for $\mathcal{T}_{J}$ is defined via a certain system of height-one ideals $\left\{H_{u} \subset \mathbb{H}_{\mathcal{F}}^{\text {n.o }}\right\}_{u \geqslant 1}$ in $\mathbb{H}_{\mathcal{F}}^{\text {n.o }}$ and might depend on the choice a priori. For a fixed natural number, we will make the following choice of a system $\left\{H_{u}\right\}_{u \geqslant 1}$ of height-one ideals:

$$
\begin{cases}\left\{H_{s}\right\}_{s} \geqslant 1=\left\{\Phi_{s}^{(j)}=\left(\gamma^{p^{s}}-\chi^{j}\left(\gamma^{p^{s}}\right)\right)\right\}_{s} \geqslant 1 & \text { in case (a), } \\ \left\{H_{t}\right\}_{t \geqslant 1}=\left\{\Psi_{t}^{(k)}=\left(\gamma^{\prime p^{t}}-\kappa^{k-2}\left(\gamma^{\prime p^{t}}\right)\right)\right\}_{t \geqslant 1} & \text { in case (b), (c) or (d). }\end{cases}
$$

We define the Bloch-Kato-type Selmer group as follows:

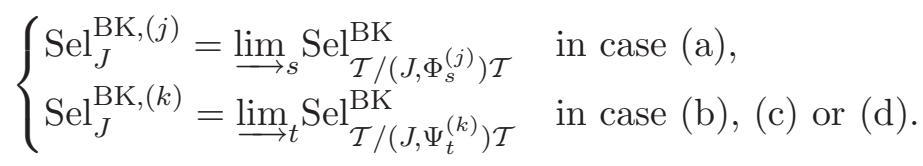

(In case $(\mathrm{a})$, we assume that $1 \leqslant j \leqslant w(\mathfrak{I})+1$.)

Let $\operatorname{Div}(M)$ be the maximal divisible subgroup for an abelian group $M$. We have the following proposition.

Proposition 4.4. We assume Condition (Ir) for $\mathcal{T}=\mathcal{T}_{\mathcal{F}}^{(i)}$ with $0 \leqslant i \leqslant p-2$. Let $J$ be a height-one ideal of $\mathbb{H}_{\mathcal{F}}^{\text {n.o }}$ determined at the beginning of $\S 4.2$ according to which of the cases (a), (b), (c) and (d) we consider. Then, we have the following.

(1) $\mathrm{Sel}_{J}^{\mathrm{BK},(l)}$ is a $\mathbb{H}_{\mathcal{F}}^{\mathrm{n} . \mathrm{o}}$-submodule of $\mathrm{Sel}_{J}^{\mathrm{Gr}}$ ( $l$ stands for $j$ or $k$ depending on which $J$ we take). The Pontryagin dual $\left(\mathrm{Sel}_{J}^{\mathrm{Gr}}\right)^{\vee}$ of $\mathrm{Sel}_{J}^{\mathrm{Gr}}$ is a finitely generated $\mathbb{H}_{\mathcal{F}}^{\text {n.o }} / J$-module. (Sel $\left.{ }_{J}^{\mathrm{Gr}}\right)^{\vee}$ is torsion over $\mathbb{H}_{\mathcal{F}}^{\text {n.o }} / J$ except in case (d) (cf. Remark 4.5).

(2) In case (a) with $J=\operatorname{Ker}(\mathfrak{I}) \mathbb{H}_{\mathcal{F}}^{\text {n.o }}$, we have

$$
\mathrm{Sel}_{J}^{\mathrm{Gr}} / \mathrm{Sel}_{J}^{\mathrm{BK},(j)} \cong \begin{cases}\left(\mathcal{O}_{\mathfrak{I}}\right)^{\vee} & \text { if } \mathrm{F}^{-} \mathcal{A}[\mathfrak{M}]^{I_{p}} \neq 0 \text { and } a_{p}\left(f_{\mathfrak{I}}\right)=1 \\ 0 & \text { if } \mathrm{F}^{-} \mathcal{A}[\mathfrak{M}]^{I_{p}}=0 \text { or } a_{p}\left(f_{\mathfrak{I}}\right) \neq 1\end{cases}
$$




\section{T. OCHIAI}

(3) We have

$$
\begin{cases}\mathrm{Sel}_{J}^{\mathrm{Gr}} / \mathrm{Sel}_{J}^{\mathrm{BK},(k)} \cong W_{J} & \text { in cases }(b) \text { and }(c), \\ \operatorname{Sel}_{J}^{\mathrm{Gr}} / \mathrm{Sel}_{J}^{\mathrm{BK},(k)} \hookrightarrow W_{J} & \text { in cases }(d),\end{cases}
$$

where

$$
W_{J}=\lim _{\longrightarrow} \frac{H_{\mathrm{Gr}}^{1}\left(\mathbb{Q}_{p}, \mathcal{A}\left[J, \Psi_{t}^{(k)}\right]\right)}{\operatorname{Div}\left(H_{\mathrm{Gr}}^{1}\left(\mathbb{Q}_{p}, \mathcal{A}\left[J, \Psi_{t}^{(k)}\right]\right)\right)} \oplus \bigoplus_{v \in \Sigma \backslash\{p, \infty\}}\left(\left(\left(\left(\mathcal{T}_{J}^{*}\right)_{I_{v}}\right)^{G_{\mathbb{Q}_{v}}}\right)_{\mathbb{H}_{\mathcal{F}} \text { ord }- \text { tor }}\right)^{\vee} .
$$

Further, the component of $W_{J}$ at each prime is given as follows.

(i) For each $v \in \Sigma \backslash\{p, \infty\}$, we have

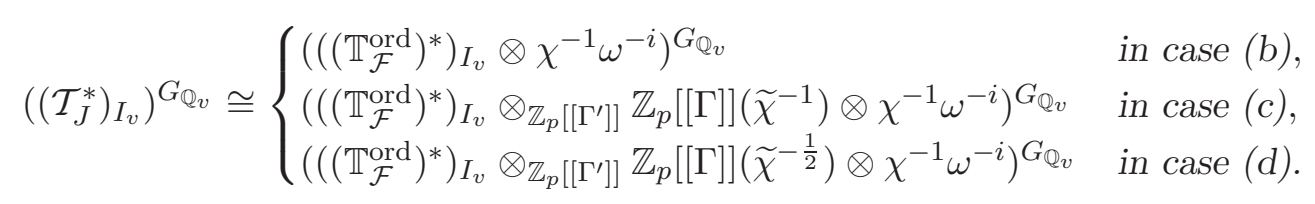

(ii) We have

$$
\lim _{\longrightarrow} \frac{H_{\mathrm{Gr}}^{1}\left(\mathbb{Q}_{p}, \mathcal{A}\left[J, \Psi_{t}^{(k)}\right]\right)}{\operatorname{Div}\left(H_{\mathrm{Gr}}^{1}\left(\mathbb{Q}_{p}, \mathcal{A}\left[J, \Psi_{t}^{(k)}\right]\right)\right)} \rightarrow H_{\mathrm{ur}}^{1}\left(\mathbb{Q}_{p}, \mathcal{A}[J]\right) .
$$

When $i \neq 1$ is satisfied or when $a_{p}\left(f_{\mathfrak{I}}\right) \neq 1$ are satisfied for every $\mathfrak{I} \in \mathfrak{X}_{\text {arith }}\left(\mathbb{H}_{\mathcal{F}}^{\text {ord }}\right)_{\geqslant 0}$, we have

We have

$$
\lim _{t} \frac{H_{\mathrm{Gr}}^{1}\left(\mathbb{Q}_{p}, \mathcal{A}\left[J, \Psi_{t}^{(k)}\right]\right)}{\operatorname{Div}\left(H_{\mathrm{Gr}}^{1}\left(\mathbb{Q}_{p}, \mathcal{A}\left[J, \Psi_{t}^{(k)}\right]\right)\right)} \cong H_{\mathrm{ur}}^{1}\left(\mathbb{Q}_{p}, \mathrm{~F}^{-} \mathcal{A}[J]\right)
$$

$$
H_{\mathrm{ur}}^{1}\left(\mathbb{Q}_{p}, \mathrm{~F}^{-} \mathcal{A}[J]\right) \cong \begin{cases}\left(\left(\left(\mathbb{H}_{\mathcal{F}}^{\text {ord }} /\left(\gamma^{\prime}-1\right)\right)\left[A_{p}(\mathcal{F})-1\right]\right)^{\vee}\right. & \text { in cases }(b) \text { and }(d) \\ 0 & \text { with } \mathrm{F}^{-} \mathcal{A}[\mathfrak{M}]^{I_{p}} \neq 0 \\ 0 & \text { otherwise. }\end{cases}
$$

Remark 4.5.

(1) In case $(\mathrm{d}),\left(\mathrm{Sel}_{J}^{\mathrm{Gr}}\right)^{\vee}$ is not necessarily a torsion $\mathbb{H}_{\mathcal{F}}^{\text {ord }}$-module. We refer the reader to $\S 7$ for more information.

(2) Let us note that $a_{p}\left(f_{\mathfrak{I}}\right)=1$ happens only when $w(\mathfrak{I})=0$ (we show this by studying the complex absolute value of $a_{p}\left(f_{\mathfrak{I}}\right)$; see, for example, [Ogg69]). In case (a), the difference in the second statement is well-known to the experts as 'trivial zero' phenomena, at least when $f_{\mathfrak{I}}$ is associated to an elliptic curve.

(3) The group $\left(\left(\left(\mathcal{T}_{J}^{*}\right)_{I_{v}}\right)^{G_{\mathbb{Q}_{v}}}\right)_{\mathbb{H}_{\mathcal{F}} \text {-ord } \text {-tor }}$ is shown to be zero if certain conditions are satisfied in case (B) of $\S 3$. In fact, we have an extension as follows in case (B):

$$
0 \longrightarrow \mathbb{H}_{\mathcal{F}}^{\text {ord }}\left(\widetilde{\chi}^{1 / 2} \chi \omega \psi\right) \longrightarrow\left(\left(\mathbb{T}_{\mathcal{F}}^{\text {ord }}\right)^{*}\right)_{I_{v}} \longrightarrow \mathbb{H}_{\mathcal{F}}^{\text {ord }}\left(\widetilde{\chi}^{1 / 2} \psi\right) /(b) \mathbb{H}_{\mathcal{F}}^{\text {ord }}\left(\widetilde{\chi}^{1 / 2} \psi\right) \longrightarrow 0,
$$

where $\psi$ is a Dirichlet character and $b \in \mathbb{H}_{\mathcal{F}}^{\text {ord }}$ is a non-zero element such that the ideal $(b)$ is prime to every height-one ideal $\Phi_{t}^{(k)}$ when $k \geqslant 2$ and $t \geqslant 0$ varies. Hence, we have

$$
\left(\left(\left(\mathcal{T}_{J}^{*}\right)_{I_{v}}\right)^{G_{\mathbb{Q}_{v}}}\right)_{\mathbb{H}_{\mathcal{F}}^{\text {ord }- \text { tor }}} \cong \begin{cases}\left(\mathbb{H}_{\mathcal{F}}^{\text {ord }}\left(\tilde{\chi}^{1 / 2} \chi^{-1} \psi \omega^{-i}\right) /(b) \mathbb{H}_{\mathcal{F}}^{\text {ord }}\left(\tilde{\chi}^{1 / 2} \chi^{-1} \psi \omega^{-i}\right)\right)^{G_{\mathbb{Q}_{v}}} & \text { in case (b), } \\ \left(\mathbb{H}_{\mathcal{F}}^{\text {ord }}\left(\tilde{\chi}^{-1 / 2} \chi^{-1} \psi \omega^{-i}\right) /(b) \mathbb{H}_{\mathcal{F}}^{\text {ord }}\left(\tilde{\chi}^{-1 / 2} \chi^{-1} \psi \omega^{-i}\right)\right)^{G_{\mathbb{Q} v}} & \text { in case (c), } \\ \left(\mathbb{H}_{\mathcal{F}}^{\text {ord }}\left(\chi^{-1} \psi \omega^{-i}\right) /(b) \mathbb{H}_{\mathcal{F}}^{\text {ord }}\left(\chi^{-1} \psi \omega^{-i}\right)\right)^{G_{\mathbb{Q}_{v}}} & \text { in case (d). }\end{cases}
$$

We see that $\left(\left(\left(\mathcal{T}_{J}^{*}\right)_{I_{v}}\right)^{G_{\mathbb{Q} v}}\right)_{\mathbb{H}_{\mathcal{F}} \text {-rord }}$ is trivial when $\psi=1$ and case (c) is satisfied or when case (d) is satisfied. We expect that $\left(\left(\left(\mathcal{T}_{J}^{*}\right)_{I_{v}}\right)^{G_{\mathbb{Q}_{v}}}\right)_{\mathbb{H}_{\mathcal{F}} \text { ord } \text {-tor }}$ is trivial in other cases. 


\section{ON THE TWO-VARIABLE IWASAWA MAIN CONJECTURE}

Proof of Proposition 4.4. Recall that $\left(\mathrm{Sel}_{J}^{\mathrm{Gr}}\right)^{\vee}$ is known to be a finitely generated torsion module over $\mathcal{O}_{\mathfrak{I}}[[\Gamma]]$ in case (a) by results of Kato and Rubin (cf. [Kat04, Rub91]). In cases (b) and (c), it is not difficult to see that $\operatorname{Sel}_{\mathcal{T} /\left(J, \Psi_{t}^{(k)}\right) \mathcal{T}}^{\mathrm{Gr}} \longrightarrow \operatorname{Sel}_{J}^{\mathrm{Gr}}\left[\Psi_{t}^{(k)}\right]$ has finite kernel and cokernel for $k \geqslant 3$ (see Proposition 5.5 and Remark 5.6 and note that $k=w(\mathfrak{I})+2$ there). If $k \geqslant 3, \mathrm{Sel}_{\mathcal{T} /\left(J, \Psi_{t}^{(k)}\right) \mathcal{T}}^{\mathrm{Gr}}$ is finite by Kato and Rubin. These facts imply that $\left(\operatorname{Sel}_{J}^{\mathrm{Gr}}\right)^{\vee} / \Psi_{t}^{(k)}\left(\mathrm{Sel}_{J}^{\mathrm{Gr}}\right)^{\vee}$ is finite. Thus, $\left(\mathrm{Sel}_{J}^{\mathrm{Gr}}\right)^{\vee}$ is a torsion $\mathbb{H}_{\mathcal{F}}^{\text {n.o }} / J \cong \mathbb{H}_{\mathcal{F}}^{\text {ord }}$-module. This completes the proof of the first assertion.

For the proof of the second and the third assertions, the following commutative diagram will play an important role.

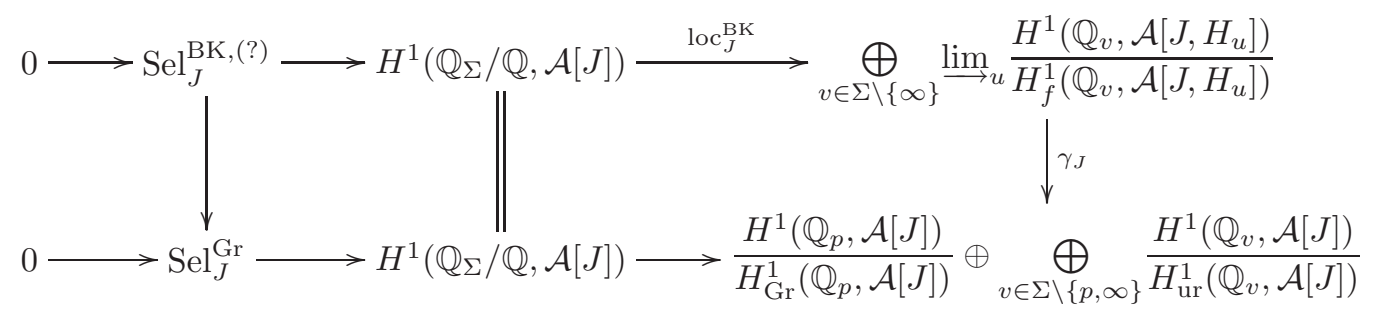

As we will see in Theorem 4.10, the map $\operatorname{loc}_{J}^{\mathrm{BK}}$ is surjective in cases (a), (b) and (c). By the snake lemma, we have

$$
\begin{cases}\operatorname{Sel}_{J}^{\mathrm{Gr}} / \operatorname{Sel}_{J}^{\mathrm{BK},(k)} \cong \operatorname{Ker}\left(\gamma_{J}\right) & \text { in cases }(\mathrm{a}),(\mathrm{b}) \text { and }(\mathrm{c}), \\ \operatorname{Sel}_{J}^{\mathrm{Gr}} / \operatorname{Sel}_{J}^{\mathrm{BK},(k)} \hookrightarrow \operatorname{Ker}\left(\gamma_{J}\right) & \text { in case }(\mathrm{d}) .\end{cases}
$$

Let us denote $\operatorname{Ker}\left(\gamma_{J}\right)$ by $W_{J}$. By Proposition 4.1,

$$
\begin{aligned}
W_{J} & \cong \lim _{\longrightarrow} \frac{H_{\mathrm{Gr}}^{1}\left(\mathbb{Q}_{p}, \mathcal{A}\left[J, H_{u}\right]\right)}{\operatorname{Div}\left(H_{\mathrm{Gr}}^{1}\left(\mathbb{Q}_{p}, \mathcal{A}\left[J, H_{u}\right]\right)\right)} \oplus \underset{v \in \Sigma \backslash\{p, \infty\}}{\bigoplus} \underset{\lim _{\longrightarrow}}{\bigoplus_{\operatorname{Div}\left(H_{\mathrm{ur}}^{1}\left(\mathbb{Q}_{v}, \mathcal{A}\left[J, H_{u}\right]\right)\right)}} \\
& \cong \lim _{\longrightarrow} \frac{H_{\mathrm{Gr}}^{1}\left(\mathbb{Q}_{p}, \mathcal{A}\left[J, H_{u}\right]\right)}{\operatorname{Div}\left(H_{\mathrm{Gr}}^{1}\left(\mathbb{Q}_{p}, \mathcal{A}\left[J, H_{u}\right]\right)\right)} \oplus \underset{v \in \Sigma \backslash\{p, \infty\}}{\bigoplus} \underset{\lim _{u}}{\bigoplus} \frac{\left(\mathcal{A}\left[J, H_{u}\right]^{I_{v}}\right)_{G_{\mathbb{Q}_{v}}}}{\operatorname{Div}\left(\left(\mathcal{A}\left[J, H_{u}\right]^{I_{v}}\right)_{G_{\mathbb{Q}_{v}}}\right)} .
\end{aligned}
$$

From (4), it suffices to calculate $W_{J}$ in each of cases (a), (b), (c) and (d). For the rest of the proof of Proposition 4.4, we restrict ourselves only to case (a). We believe that this restriction is better to keep the proof at a reasonable length. The proof is basically the same in the other cases (b), (c) and (d). So we will also avoid unnecessarily complicated notation caused by unified treatment which covers every case.

In case (a), with $J=\operatorname{Ker}(\mathfrak{I}) \mathbb{H}_{\mathcal{F}}^{\text {n.o }}, u$ and $H_{u}$ correspond to $s$ and $\Phi_{s}^{(j)}$ respectively for $j$ a fixed number with $1 \leqslant j \leqslant w(\mathfrak{I})+1)$. The Pontryagin dual of

$$
\lim _{\rightarrow} \frac{\left(\mathcal{A}\left[J, \Phi_{s}^{(j)}\right]^{I_{v}}\right)_{G_{\mathbb{Q}_{v}}}}{\operatorname{Div}\left(\left(\mathcal{A}\left[J, \Phi_{s}^{(j)}\right]^{I_{v}}\right)_{G_{\mathbb{Q}_{v}}}\right)}
$$

in the term of $W_{J}$ outside $p$ is $\lim _{s}\left(\left(\left(\mathcal{T}_{J}^{*}\right)_{I_{v}} / \Phi_{s}^{(j)}\left(\mathcal{T}_{J}^{*}\right)_{I_{v}}\right)^{G_{\mathbb{Q}_{v}}}\right)_{\mathbb{Z}_{p} \text {-tor }}$ at each $v \in \Sigma \backslash\{p, \infty\}$, where $(\cdot)_{\mathbb{Z}_{p} \text {-tor }}$ means the torsion-part as a $\mathbb{Z}_{p}$-module and $\mathcal{T}_{J}^{*}=\operatorname{Hom}_{\mathbb{H}_{\mathcal{F}}^{\text {n.o }} / J}\left(\mathcal{T}_{J}, \mathbb{H}_{\mathcal{F}}^{\text {n.o }} / J\right)$. We have

$$
\left(\left(\mathcal{T}_{J}^{*}\right)_{I_{v}}\right)_{\left(\mathbb{H}_{\mathcal{F}}^{\text {n.o }} / J\right) \text {-tor }} \cong\left(\left(T_{f_{\mathfrak{I}}}^{*}\right)_{I_{v}}\right)_{\mathbb{Z}_{p} \text {-tor }} \otimes_{\mathbb{Z}_{p}} \mathbb{Z}_{p}[[\Gamma]]\left(\tilde{\chi}^{-1}\right)
$$

since $\mathcal{T}_{J}^{*}$ is isomorphic to $T_{f_{\mathfrak{I}}}^{*} \otimes_{\mathbb{Z}_{p}} \mathbb{Z}_{p}[[\Gamma]]\left(\tilde{\chi}^{-1}\right)$, where $T_{f_{\mathfrak{I}}}^{*}$ means $\operatorname{Hom}_{\mathbb{Z}_{p}}\left(T_{f_{\mathfrak{I}}}, \mathbb{Z}_{p}\right)$. In $(5)$, $\left(\left(T_{f_{\mathfrak{I}}}^{*}\right)_{I_{v}}\right)_{\mathbb{Z}_{p} \text {-tor }}$ is a finite abelian group and the action of $G_{\mathbb{Q}_{v}}$ on $\mathbb{Z}_{p} /\left(p^{n}\right)[[\Gamma]]\left(\tilde{\chi}^{-1}\right)$ is not finite for any $n$. Hence, $\left(\left(\left(\mathcal{T}_{J}^{*}\right)_{I_{v}}\right)_{\left(\mathbb{H}_{\mathcal{F}}^{\text {n.o }} / J\right) \text {-tor }}\right)^{G_{\mathbb{Q} v}}$ must be zero. The proof for the contribution of local terms outside $p$ in case (a) is completed. In cases (b), (c) and (d), we also calculate the contribution outside $p$ by using results in $\S 3$, but we omit the proof for the reason mentioned earlier and we only 


\section{T. OCHIAI}

refer the reader to Theorem 3.3 for the structure of $\left(\mathcal{T}_{J}^{*}\right)_{I_{v}}$ (note that $\left(\mathcal{T}_{J}^{*}\right)_{I_{v}}$ has an isomorphism with $\left(\left(\mathbb{T}^{\text {ord }}\right)^{*}\right)_{I_{v}}$ over $\mathbb{H}_{\mathcal{F}}^{\text {ord }}$ which is not necessarily compatible as a $G_{\mathbb{Q}_{v}}$-module).

Next, we discuss the group

$$
\lim _{\rightarrow} \frac{H_{\mathrm{Gr}}^{1}\left(\mathbb{Q}_{p}, \mathcal{A}\left[J, \Phi_{s}^{(j)}\right]\right)}{\operatorname{Div}\left(H_{\mathrm{Gr}}^{1}\left(\mathbb{Q}_{p}, \mathcal{A}\left[J, \Phi_{s}^{(j)}\right]\right)\right)},
$$

which is the $p$-part of $W_{J}$. By definition, we have the exact sequence

$$
0 \longrightarrow H_{\mathrm{Gr}}^{1}\left(\mathbb{Q}_{p}, \mathcal{A}\left[J, \Phi_{s}^{(j)}\right]\right) \longrightarrow H^{1}\left(\mathbb{Q}_{p}, \mathcal{A}\left[J, \Phi_{s}^{(j)}\right]\right) \stackrel{a_{s}}{\longrightarrow} H^{1}\left(I_{p}, \mathrm{~F}^{-} \mathcal{A}\left[J, \Phi_{s}^{(j)}\right]\right)^{G_{\mathbb{Q}_{p}}},
$$

where the latter map $a_{s}$ is decomposed as follows for each $s$ :

$$
H^{1}\left(\mathbb{Q}_{p}, \mathcal{A}\left[J, \Phi_{s}^{(j)}\right]\right) \stackrel{a_{s}^{\prime}}{\longrightarrow} H^{1}\left(\mathbb{Q}_{p}, \mathrm{~F}^{-} \mathcal{A}\left[J, \Phi_{s}^{(j)}\right]\right) \stackrel{a_{s}^{\prime \prime}}{\longrightarrow} H^{1}\left(I_{p}, \mathrm{~F}^{-} \mathcal{A}\left[J, \Phi_{s}^{(j)}\right]\right)^{G_{\mathbb{Q}_{p}}} .
$$

Hence, we have the following extension:

$$
\lim _{s} \frac{\operatorname{Ker}\left(a_{s}^{\prime}\right)}{\operatorname{Div}\left(\operatorname{Ker}\left(a_{s}^{\prime}\right)\right)} \longrightarrow \lim _{s} \frac{H_{\mathrm{Gr}}^{1}\left(\mathbb{Q}_{p}, \mathcal{A}\left[J, \Phi_{s}^{(j)}\right]\right)}{\operatorname{Div}\left(H_{\mathrm{Gr}}^{1}\left(\mathbb{Q}_{p}, \mathcal{A}\left[J, \Phi_{s}^{(j)}\right]\right)\right)} \longrightarrow \lim _{s} \frac{\operatorname{Ker}\left(a_{s}^{\prime \prime}\right) \cap \operatorname{Im}\left(a_{s}^{\prime}\right)}{\operatorname{Div}\left(\operatorname{Ker}\left(a_{s}^{\prime \prime}\right) \cap \operatorname{Im}\left(a_{s}^{\prime}\right)\right)} \longrightarrow 0 .
$$

The first group

is a quotient of

$$
\frac{\operatorname{Ker}\left(a_{s}^{\prime}\right)}{\operatorname{Div}\left(\operatorname{Ker}\left(a_{s}^{\prime}\right)\right)}
$$

$$
\frac{H^{1}\left(\mathbb{Q}_{p}, \mathrm{~F}^{+} \mathcal{A}\left[J, \Phi_{s}^{(j)}\right]\right)}{\operatorname{Div}\left(H^{1}\left(\mathbb{Q}_{p}, \mathrm{~F}^{+} \mathcal{A}\left[J, \Phi_{s}^{(j)}\right]\right)\right)}
$$

and we have

$$
\begin{aligned}
\frac{H^{1}\left(\mathbb{Q}_{p}, \mathrm{~F}^{+} \mathcal{A}\left[J, \Phi_{s}^{(j)}\right]\right)}{\operatorname{Div}\left(H^{1}\left(\mathbb{Q}_{p}, \mathrm{~F}^{+} \mathcal{A}\left[J, \Phi_{s}^{(j)}\right]\right)\right)} & \cong H^{2}\left(\mathbb{Q}_{p}, \mathrm{~F}^{+} \mathcal{T}_{J} / \Phi_{s}^{(j)} \mathrm{F}^{+} \mathcal{T}_{J}\right)_{\mathbb{Z}_{p} \text {-tor }} \\
& \cong\left(\left(\mathrm{F}^{+} \mathcal{T}_{J}(-1)\right)_{G_{\mathbb{Q}_{p}}} / \Phi_{s}^{(j)}\left(\mathrm{F}^{+} \mathcal{T}_{J}(-1)\right)_{G_{\mathbb{Q}_{p}}}\right)_{\mathbb{Z}_{p} \text {-tor }}
\end{aligned}
$$

In case (a) with $J=\operatorname{Ker}(\mathfrak{I}) \mathbb{H}_{\mathcal{F}}^{\text {n.o }}$, we have

$$
\mathrm{F}^{+} \mathcal{T}_{J}(-1) \cong \mathcal{O}_{\mathfrak{I}}[[\Gamma]]\left(\omega^{i-1} \chi^{-1} \tilde{\chi}\right) \otimes_{\mathcal{O}_{\mathfrak{I}}} \mathcal{O}_{\mathfrak{I}}\left(\alpha_{\mathfrak{I}}\right),
$$

where $\mathcal{O}_{\mathfrak{I}}[[\Gamma]]\left(\omega^{i-1} \chi^{-1} \tilde{\chi}\right)$ is a free $\mathcal{O}_{\mathfrak{I}}[[\Gamma]]$-module of rank one on which $G_{\mathbb{Q}_{p}}$ acts via $\omega^{i-1} \chi^{-1} \tilde{\chi}$ and $\mathcal{O}_{\mathfrak{I}}\left(\alpha_{\mathfrak{I}}\right)$ is a free $\mathcal{O}_{\mathfrak{I}}$-module of rank one on which $G_{\mathbb{Q}_{p}}$ acts via the unramified character $\alpha_{\mathfrak{I}}$ : $G_{\mathbb{Q}_{p}} \longrightarrow \mathcal{O}_{\mathfrak{I}}^{\times}$given by $\alpha_{\mathfrak{I}}\left(\operatorname{Frob}_{p}\right)=a_{p}\left(f_{\mathfrak{I}}\right)$. Since $I_{p}$ acts on $\mathrm{F}^{+} \mathcal{T}_{J}(-1) / \mathfrak{M F}^{+} \mathcal{T}_{J}(-1)$ via $\omega^{i-1}$, we have

$$
\begin{cases}\left(\mathrm{F}^{+} \mathcal{T}_{J}(-1)\right)_{G_{\mathbb{Q}_{p}}} / \Phi_{s}^{(j)}\left(\mathrm{F}^{+} \mathcal{T}_{J}(-1)\right)_{G_{\mathbb{Q} p}}=0 & i \neq 1 \\ \left(\mathrm{~F}^{+} \mathcal{T}_{J}(-1)\right)_{G_{\mathbb{Q}_{p}}} / \Phi_{s}^{(j)}\left(\mathrm{F}^{+} \mathcal{T}_{J}(-1)\right)_{G_{\mathbb{Q} p}} \cong \mathcal{O}_{\mathfrak{J}} /\left(a_{p}\left(f_{\mathfrak{I}}\right)-1\right) & i=1\end{cases}
$$

We recall the following lemma.

Lemma 4.6. Let $M$ be a finite $\mathbb{H}_{\mathcal{F}}^{\text {n.o }} / J$-module. Then we have $\lim _{u}\left(M / \Phi_{s}^{(j)} M\right)=0$ (note that $\mathbb{H}_{\mathcal{F}}^{\text {n.o }} / J$ is isomorphic to $\left.\mathcal{O}_{\mathfrak{I}}[[\Gamma]]\right)$.

We omit the proof of Lemma 4.6 and we only refer the reader to [Och03, Lemma 4.7]. We prove a similar result for modules over a two-variable Iwasawa algebra there, but the proof is the same as the case of a one-variable Iwasawa algebra in Lemma 4.6. By Lemma 4.6, we have

$$
\lim _{s}\left(\left(\mathrm{~F}^{+} \mathcal{T}_{J}(-1)\right)_{G_{\mathbb{Q} p}} / \Phi_{s}^{(j)}\left(\mathrm{F}^{+} \mathcal{T}_{J}(-1)\right)_{G_{\mathbb{Q}_{p}}}\right)_{\mathbb{Z}_{p} \text {-tor }}=0
$$




\section{ON THE TWO-VARIABLE IWASAWA MAIN CONJECTURE}

Hence,

in (6) is trivial.

$$
\lim _{s} \frac{\operatorname{Ker}\left(a_{s}^{\prime}\right)}{\operatorname{Div}\left(\operatorname{Ker}\left(a_{s}^{\prime}\right)\right)}
$$

For the proof of Proposition 4.4, we need to show that

$$
\lim _{s} \frac{\operatorname{Ker}\left(a_{s}^{\prime \prime}\right) \cap \operatorname{Im}\left(a_{s}^{\prime}\right)}{\operatorname{Div}\left(\operatorname{Ker}\left(a_{s}^{\prime \prime}\right) \cap \operatorname{Im}\left(a_{s}^{\prime}\right)\right)} \cong H_{\mathrm{ur}}^{1}\left(\mathbb{Q}_{p}, \mathrm{~F}^{-} \mathcal{A}[J]\right) .
$$

We have the following claims.

Claim 4.7. The module $\operatorname{Ker}\left(a_{s}^{\prime \prime}\right) \cap \operatorname{Im}\left(a_{s}^{\prime}\right)$ is finite for every $s$.

Claim 4.8. For any height-one ideal $I \subset \mathbb{H}_{\mathcal{F}}^{\text {n.o }}, H^{1}\left(\mathbb{Q}_{p}, \mathcal{A}[I]\right) \stackrel{a}{\longrightarrow} H^{1}\left(\mathbb{Q}_{p}, \mathrm{~F}^{-} \mathcal{A}[I]\right)$ is surjective.

We will finish the proof of Proposition 4.4 by using these claims. By Claim 4.7, we have

$$
\lim _{s} \frac{\operatorname{Ker}\left(a_{s}^{\prime \prime}\right) \cap \operatorname{Im}\left(a_{s}^{\prime}\right)}{\operatorname{Div}\left(\operatorname{Ker}\left(a_{s}^{\prime \prime}\right) \cap \operatorname{Im}\left(a_{s}^{\prime}\right)\right)} \cong \lim _{s} \operatorname{Ker}\left(a_{s}^{\prime \prime}\right) \cap \operatorname{Im}\left(a_{s}^{\prime}\right) .
$$

By Claim 4.8, we have

$$
\lim _{s} \operatorname{Ker}\left(a_{s}^{\prime \prime}\right) \cap \operatorname{Im}\left(a_{s}^{\prime}\right) \cong \lim _{s} \operatorname{Ker}\left(a_{s}^{\prime \prime}\right) \cong H_{\mathrm{ur}}^{1}\left(\mathbb{Q}_{p}, \mathrm{~F}^{-} \mathcal{A}[J]\right) .
$$

This completes the proof of (7). Let us finally calculate the group $H_{\mathrm{ur}}^{1}\left(\mathbb{Q}_{p}, \mathrm{~F}^{-} \mathcal{A}[J]\right)$. In case (a) with $J=\operatorname{Ker}(\mathfrak{I}) \mathbb{H}_{\mathcal{F}}^{\text {n.o }}$, we have

$$
\mathrm{F}^{-} \mathcal{A}[J]^{I_{p}} \cong \begin{cases}\left(\mathcal{O}_{\mathfrak{I}}\right)^{\vee} & \text { if } \mathrm{F}^{-} \mathcal{A}[\mathfrak{M}]^{I_{p}} \neq 0 \\ 0 & \text { if } \mathrm{F}^{-} \mathcal{A}[\mathfrak{M}]^{I_{p}}=0\end{cases}
$$

on which $\operatorname{Frob}_{p} \in G_{\mathbb{Q}_{p}} / I_{p}$ acts via multiplication by $a_{p}\left(f_{\mathfrak{I}}\right)^{-1}$.

In the rest of the proof, we finish the proof of the two claims above.

Proof of Claim 4.7. In case (a) with $J=\operatorname{Ker}(\mathfrak{I}) \mathbb{H}_{\mathcal{F}}^{\text {n.o }}$, it suffices to show that

$$
\operatorname{Im}\left[H^{1}\left(\mathbb{Q}_{p}, V_{J, \Phi_{s}^{(j)}}\right) \longrightarrow H^{1}\left(\mathbb{Q}_{p}, \mathrm{~F}^{-} V_{J, \Phi_{s}^{(j)}}\right)\right] \cap H_{\mathrm{ur}}^{1}\left(\mathbb{Q}_{p}, \mathrm{~F}^{-} V_{J, \Phi_{s}^{(j)}}\right)=0
$$

for every $s$, since we have

$$
V_{J, \Phi_{s}^{(j)}}=\left(V_{f_{\mathfrak{I}}} \otimes \chi^{j} \omega^{i}\right) \otimes_{\mathbb{Z}_{p}} \mathbb{Z}_{p}\left[\Gamma / \Gamma^{p^{s}}\right](\tilde{\chi}),
$$

by definition and since the inertia group $I_{p}$ acts on $\mathrm{F}^{-} V_{f_{\mathfrak{I}}} \otimes \chi^{j} \omega^{i}$ via the character $\chi^{j-1-w(\mathfrak{I})}$ modulo a finite character. Thus, we have

$$
\left(\mathrm{F}^{-} V_{J, \Phi_{s}^{(j)}}\right)^{I_{p}}= \begin{cases}0 & \text { if } j \neq w(\mathfrak{I})+1 \text { or }\left(\mathrm{F}^{-} \mathcal{A}[\mathfrak{M}] \otimes \omega^{i}\right)^{I_{p}}=0, \\ K_{\mathfrak{I}}\left(\alpha_{\mathfrak{I}}^{-1}\right) & \text { otherwise, }\end{cases}
$$

where $K_{\mathfrak{I}}\left(\alpha_{\mathfrak{I}}^{-1}\right)$ is a vector space of rank one over $K_{\mathfrak{I}}=\mathcal{O}_{\mathfrak{I}} \otimes_{\mathbb{Z}_{p}} \mathbb{Q}_{p}$ on which $G_{\mathbb{Q}_{p}}$ acts via $\alpha_{\mathfrak{I}}^{-1}$. This implies that $H_{\mathrm{ur}}^{1}\left(\mathbb{Q}_{p}, \mathrm{~F}^{-} V_{J, \Phi_{s}^{(j)}}\right)=\left(\left(\mathrm{F}^{-} V_{J, \Phi_{s}^{(j)}}\right)^{I_{p}}\right)_{G_{\mathbb{Q}_{p}}}=0$ if $a_{p}\left(f_{\mathfrak{I}}\right) \neq 1$ and our proof is completed in this case. Now, let us suppose that $a_{p}\left(f_{\mathfrak{I}}\right)=1$. This happens only when $w(\mathfrak{I})=0$ (cf. Remark 4.5(2)). Note that $w(\mathfrak{I})=0$ implies $j=1$ and $V_{f_{\mathfrak{I}}} \subset H_{\text {ét }}^{1}\left(B_{\mathfrak{I}} \otimes_{\mathbb{Q}} \overline{\mathbb{Q}}, \mathbb{Q}_{p}\right)$ for certain abelian variety $B_{\mathfrak{I}}$ over $\mathbb{Q}$. Let $\mathbb{Q}_{f_{\mathfrak{I}}}$ be a finite extension of $\mathbb{Q}$ obtained by adjoining all Fourier coefficients $a_{n}\left(f_{\mathfrak{I}}\right)$ of $f_{\mathfrak{I}}$. The field $K_{\mathfrak{I}}$ is naturally identified with a direct-summand of $\mathbb{Q}_{f_{\mathfrak{I}}} \otimes_{\mathbb{Q}} \mathbb{Q}_{p}$. Since $a_{p}\left(f_{\mathfrak{I}}\right)=1$, there exists an abelian variety $B_{\mathfrak{I}}^{\prime}$ over $\mathbb{Q}_{p}$ with the following properties.

(1) $B_{\mathfrak{I}}^{\prime}$ is isogenious to a sub-abelian variety of $B_{\mathfrak{I}} \otimes \mathbb{Q}_{p}$ of dimension $d=\left[K_{\mathfrak{I}}: \mathbb{Q}_{p}\right]$ over $\mathbb{Q}_{p}$.

(2) $B_{\mathfrak{J}}^{\prime}$ has totally multiplicative reduction over $\mathbb{Q}_{p}$.

(3) $H_{\text {ét }}^{1}\left(B_{\mathfrak{I}}^{\prime} \otimes_{\mathbb{Q}_{p}} \overline{\mathbb{Q}}_{p}, \mathbb{Q}_{p}\right)$ is isomorphic to $V_{f_{\mathfrak{I}}}$ as a $G_{\mathbb{Q}_{p}}$-module. 


\section{T. OCHIAI}

By the third property, $V_{f_{\mathfrak{I}}} \otimes \chi \omega \cong V_{p}\left(B_{\mathfrak{I}}^{\prime \mathrm{t}}\right):=T_{p}\left(B_{\mathfrak{I}}^{\prime \mathrm{t}}\right) \otimes_{\mathbb{Z}_{p}} \mathbb{Q}_{p}$, where $B_{\mathfrak{I}}^{\prime \mathrm{t}}$ is the dual abelian variety of $B_{\mathfrak{I}}^{\prime}$ and $T_{p}\left(B_{\mathfrak{I}}^{\prime t}\right)$ is the $p$-Tate module $\varliminf_{n} B_{\mathfrak{I}}^{\prime t}\left(\overline{\mathbb{Q}}_{p}\right)\left[p^{n}\right]$. Since $B_{\mathfrak{I}}^{\prime t}$ has totally multiplicative reduction over $\mathbb{Q}_{p}, B_{\mathfrak{J}}^{\prime \mathrm{t}}\left(\overline{\mathbb{Q}}_{p}\right)$ is isomorphic to $\left(\overline{\mathbb{Q}}_{p}^{\times}\right)^{d} / P$ as $G_{\mathbb{Q}_{p}}$-module using Tate's uniformization of $B_{\mathfrak{I}}^{\prime}$ when $d=1$ or its generalization by Mumford (cf. [FC90, Appendix]) when $d>1$, where $P$ is subgroup of $\left(\overline{\mathbb{Q}}_{p}^{\times}\right)^{d}$ which is mapped into a free $\mathbb{Z}$-module of rank $d$ in $\mathbb{Q}^{\oplus d}$ via the composite $P \hookrightarrow\left(\overline{\mathbb{Q}}_{p}^{\times}\right)^{d} \stackrel{\operatorname{ord}_{p}}{\longrightarrow} \mathbb{Q}^{\oplus d}$. Since $a_{p}\left(f_{\mathfrak{I}}\right)=1, \alpha_{\mathfrak{I}}$ is a trivial character. Hence, $P$ is contained in $\left(\mathbb{Q}_{p}^{\times}\right)^{d}$ and $\mathrm{F}^{+} V_{p}\left(B_{\mathfrak{I}}^{\prime \mathrm{t}}\right):=\left(\mathrm{F}^{+} V_{f_{\mathfrak{I}}} \otimes \chi \omega\right) \cap V_{p}\left(B_{\mathfrak{I}}^{\prime \mathrm{t}}\right)\left(\right.$ respectively $\left.\mathrm{F}^{-} V_{p}\left(B_{\mathfrak{I}}^{\prime \mathrm{t}}\right):=V_{p}\left(B_{\mathfrak{I}}^{\prime \mathrm{t}}\right) / \mathrm{F}^{+} V_{p}\left(B_{\mathfrak{I}}^{\prime \mathrm{t}}\right)\right)$ is isomorphic to $\mathbb{Q}_{p}(\chi \omega)^{\oplus d}$ (respectively $\mathbb{Q}_{p}^{\oplus d}$ ). By Shapiro's lemma on induced Galois representations, we have:

$$
\begin{aligned}
& \operatorname{Im}\left[H^{1}\left(\mathbb{Q}_{p}, V_{J, \Phi_{s}^{(1)}}\right) \longrightarrow H^{1}\left(\mathbb{Q}_{p}, \mathrm{~F}^{-} V_{J, \Phi_{s}^{(1)}}\right)\right] \cap H_{\mathrm{ur}}^{1}\left(\mathbb{Q}_{p}, \mathrm{~F}^{-} V_{J, \Phi_{s}^{(1)}}\right) \\
& \quad=\operatorname{Im}\left[H^{1}\left(\mathbb{Q}_{p, s}, V_{p}\left(B_{\mathfrak{I}}^{\prime \mathrm{t}}\right)\right) \longrightarrow H^{1}\left(\mathbb{Q}_{p, s}, \mathrm{~F}^{-} V_{p}\left(B_{\mathfrak{I}}^{\prime \mathrm{t}}\right)\right)\right] \cap H_{\mathrm{ur}}^{1}\left(\mathbb{Q}_{p, s}, \mathrm{~F}^{-} V_{p}\left(B_{\mathfrak{I}}^{\prime \mathrm{t}}\right)\right)
\end{aligned}
$$

where $\mathbb{Q}_{p, s}$ is the unique Galois extension of $\mathbb{Q}_{p}$ contained in $\mathbb{Q}_{p}\left(\mu_{p^{s+1}}\right)$ with $\operatorname{Gal}\left(\mathbb{Q}_{p, s} / \mathbb{Q}_{p}\right) \cong \mathbb{Z} /\left(p^{s}\right)$. By the properties of $P$ mentioned above, we have $P \cong \prod_{1 \leqslant h \leqslant d} q_{h}^{\mathbb{Z}} \subset\left(\mathbb{Q}_{p}^{\times}\right)^{d}$ with $\operatorname{ord}_{p}\left(q_{h}\right)>0$ for each $1 \leqslant i \leqslant d$. Via the identification

$$
H^{1}\left(\mathbb{Q}_{p, s}, \mathrm{~F}^{-} V_{p}\left(B_{\mathfrak{I}}^{\prime t}\right)\right) \cong H^{1}\left(\mathbb{Q}_{p, s}, \mathbb{Q}_{p}^{\oplus d}\right) \cong \bigoplus_{1 \leqslant h \leqslant d} \operatorname{Hom}\left(G_{\mathbb{Q}_{p, s}}, \mathbb{Q}_{p}\right)
$$

the image of $H^{1}\left(\mathbb{Q}_{p, s}, V_{p}\left(B_{\mathfrak{I}}^{\prime t}\right)\right) \longrightarrow H^{1}\left(\mathbb{Q}_{p, s}, \mathrm{~F}^{-} V_{p}\left(B_{\mathfrak{I}}^{\prime t}\right)\right)$ is equal to:

$$
\bigoplus_{1 \leqslant h \leqslant d} \operatorname{Hom}\left(\operatorname{Gal}\left(F_{\infty, s}^{(h)} / \mathbb{Q}_{p, s}\right), \mathbb{Q}_{p}\right) \subset \bigoplus_{1 \leqslant h \leqslant d} \operatorname{Hom}\left(G_{\mathbb{Q}_{p, s}}, \mathbb{Q}_{p}\right)
$$

where $F_{\infty, s}^{(h)} / \mathbb{Q}_{p, s}$ is the Galois extension of $\mathbb{Q}_{p, s}$ characterized as follows.

(1) $\operatorname{Gal}\left(F_{\infty, s}^{(h)} / \mathbb{Q}_{p, s}\right)$ is isomorphic to $\mathbb{Z}_{p}$.

(2) The universal norm $\bigcap_{\mathbb{Q}_{p, s} \subset F \subset F_{\infty, s}^{(h)}} \operatorname{Norm}_{F / \mathbb{Q}_{p, s}}\left(F^{\times}\right)$coincides with $\mu_{p-1} \cdot q_{h}^{\mathbb{Z}} \subset \mathbb{Q}_{p, s}^{\times}$.

Since we have

$$
\begin{aligned}
& \left(\bigoplus_{1 \leqslant h \leqslant d} \operatorname{Hom}\left(\operatorname{Gal}\left(F_{\infty, s}^{(h)} / \mathbb{Q}_{p, s}\right), \mathbb{Q}_{p}\right)\right) \cap H_{\mathrm{ur}}^{1}\left(\mathbb{Q}_{p, s}, \mathbb{Q}_{p}^{\oplus d}\right) \\
& \quad=\left(\bigoplus_{1 \leqslant h \leqslant d} \operatorname{Hom}\left(\operatorname{Gal}\left(F_{\infty, s}^{(h)} / \mathbb{Q}_{p, s}\right), \mathbb{Q}_{p}\right)\right) \cap \operatorname{Hom}\left(\operatorname{Gal}\left(\mathbb{Q}_{p, s}^{\mathrm{ur}} / \mathbb{Q}_{p, s}\right), \mathbb{Q}_{p}^{\oplus d}\right)=0
\end{aligned}
$$

we have completed the proof in case (a).

Proof of Claim 4.8. The cokernel of $a$ is a submodule of $H^{2}\left(\mathbb{Q}_{p}, \mathrm{~F}^{+} \mathcal{A}[J]\right)$, which is the Pontryagin dual of $\left(\left(\left(\mathrm{F}^{+} \mathcal{T}\right)^{*}(-1)\right)_{J}\right)^{G_{\mathbb{Q}_{p}}}$. Since $\left(\left(\mathrm{F}^{+} \mathcal{T}\right)^{*}(-1)\right)_{G_{\mathbb{Q} p}}$ has support whose codimension is greater than or equal to two, $\left(\left(\left(\mathrm{F}^{+} \mathcal{T}\right)^{*}(-1)\right)_{J}\right)^{G_{\mathbb{Q}_{p}}}$ must be zero for any height-one prime $J \subset \mathbb{H}_{\mathcal{F}}^{\text {n.o }}$. Consequently, the map $a$ is surjective. This completes the proof of Claim 4.8.

This completes the proof of Proposition 4.4

\subsection{Selmer groups over the two-variable Iwasawa algebra}

For each $j, k$ with $1 \leqslant j \leqslant k-1$, we define $\operatorname{Sel}_{\mathcal{T}}^{\mathrm{Gr}} \subset H^{1}\left(\mathbb{Q}_{\Sigma} / \mathbb{Q}, \mathcal{A}\right)$ in the same way as above by using

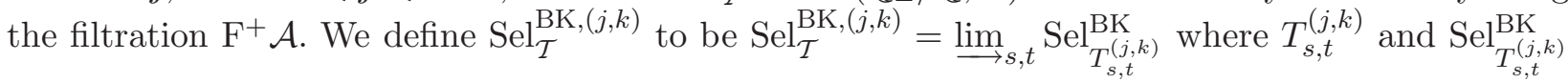
are as given in $\S$ 4.1. A priori, $\operatorname{Sel}_{\mathcal{T}}^{\mathrm{BK},(j, k)}$ might depend on the choice of $(j, k)$. However, we have the following proposition. 


\section{ON THE TWO-VARIABLE IWASAWA MAIN CONJECTURE}

Proposition 4.9. Assume Condition (Ir) above. We have the following statements.

(1) Selmer groups $\operatorname{Sel}_{\mathcal{T}}^{\mathrm{Gr}}$ and $\operatorname{Sel}_{\mathcal{T}}^{\mathrm{BK},(j, k)}$ are equal as subgroups of $H^{1}\left(\mathbb{Q}_{\Sigma} / \mathbb{Q}, \mathcal{A}\right)$. In particular, the definition of $\operatorname{Sel}_{\mathcal{T}}^{\mathrm{BK},(j, k)}$ does not depend on the choice of $(j, k)$ as above.

(2) The Pontryagin dual of $\left(\operatorname{Sel}_{\mathcal{T}}^{\mathrm{Gr}}\right)^{\vee}$ is a torsion module over $\mathbb{H}_{\mathcal{F}}^{\text {n.o }}$.

Proof. The first statement is implicitly proved in [Och03]. We recall briefly how to use the result in [Och03]. Recall the following diagram.

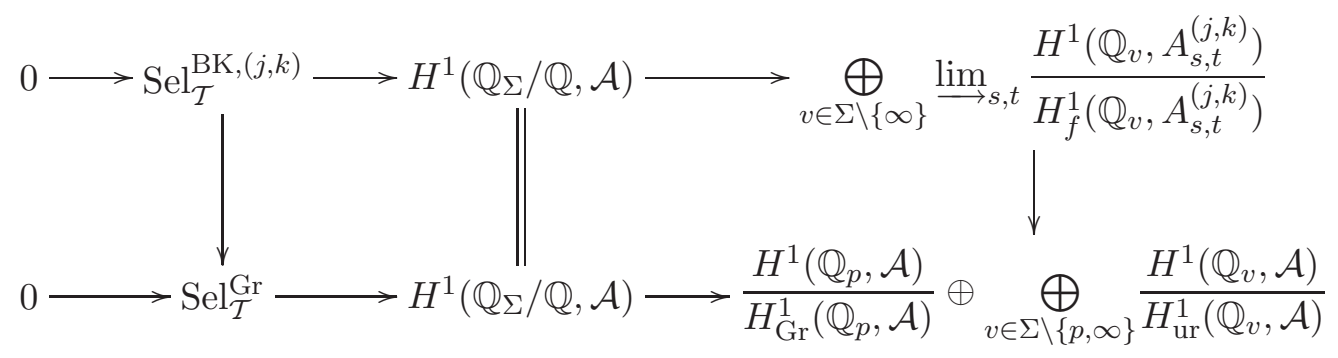

For $v \in \Sigma \backslash\{p, \infty\}, \lim _{s, t} H_{f}^{1}\left(\mathbb{Q}_{v}, A_{s, t}^{(j, k)}\right)$ is a subgroup of $H_{\mathrm{ur}}^{1}\left(\mathbb{Q}_{v}, \mathcal{A}\right)$ by definition. We have $H_{\mathrm{ur}}^{1}\left(\mathbb{Q}_{v}, \mathcal{A}\right) \cong H^{1}\left(\mathbb{Q}_{v}^{\text {ur }} / \mathbb{Q}_{v}, \mathcal{A}^{I_{v}}\right)$ by the inflation-restriction sequence. By Shapiro's lemma, $H^{1}\left(\mathbb{Q}_{v}^{\text {ur }} / \mathbb{Q}_{v}, \mathcal{A}^{I_{v}}\right)$ is isomorphic to $H^{1}\left(\mathbb{Q}_{v}^{\text {ur }} / \mathbb{Q}_{v, \infty},(\mathcal{A}[\gamma-1])^{I_{v}}\right)$. Here $\mathbb{Q}_{v, \infty}$ is the unique sub-extension of $\mathbb{Q}_{v}\left(\mu_{p^{\infty}}\right) / \mathbb{Q}_{v}$ such that $\operatorname{Gal}\left(\mathbb{Q}_{v, \infty} / \mathbb{Q}_{v}\right) \cong \mathbb{Z}_{p}$. Note that $\operatorname{Gal}\left(\mathbb{Q}_{v}^{\text {ur }} / \mathbb{Q}_{v, \infty}\right)$ is isomorphic to $\prod_{l \neq p} \mathbb{Z}_{l}$ and that $(\mathcal{A}[\gamma-1])^{I_{v}}$ is a $p$-torsion group. Hence, we have $\lim _{s, t} H_{f}^{1}\left(\mathbb{Q}_{v}, A_{s, t}^{(j, k)}\right)=H_{\mathrm{ur}}^{1}\left(\mathbb{Q}_{v}, \mathcal{A}\right)=0$ for any $v \in \Sigma \backslash\{p, \infty\}$. On the other hand, $\lim _{s, t} H_{f}^{1}\left(\mathbb{Q}_{p}, A_{s, t}^{(j, k)}\right)=H_{\mathrm{Gr}}^{1}\left(\mathbb{Q}_{p}, \mathcal{A}\right)$ by [Och03, Corollary 4.13]. This completes the proof of the first assertion.

The proof of the second assertion is basically the same as that of Proposition 4.4(1). We apply Proposition 5.2 to compare $\left(\operatorname{Sel}_{\mathcal{T}}^{\mathrm{Gr}}\right)^{\vee} / J\left(\operatorname{Sel}_{\mathcal{T}}^{\mathrm{Gr}}\right)^{\vee}$ with $\left(\operatorname{Sel}_{J}^{\mathrm{Gr}}\right)^{\vee}$ and we apply inductively the result obtained in Proposition 4.4(1) that $\left(\operatorname{Sel}_{J}^{\mathrm{Gr}}\right) \vee$ is a torsion $\mathbb{H}_{\mathcal{F}}^{\text {n.o }} / J$-module. Thus, we show that $\left(\operatorname{Sel}_{\mathcal{T}}^{\mathrm{Gr}}\right)^{\vee}$ is a torsion $\mathbb{H}_{\mathcal{F}}^{\text {n.o }}$-module.

Remark on the notation. By Proposition 4.9(1), $\operatorname{Sel}_{\mathcal{T}}^{\mathrm{Gr}}$ and $\operatorname{Sel}_{\mathcal{T}}^{\mathrm{BK}}$ coincide with each other for a two-variable nearly ordinary deformation $\mathcal{T}$. Hence, we denote the Selmer group for $\mathcal{T}$ by $\operatorname{Sel}_{\mathcal{T}}$ from now on. For various specializations $\mathcal{T}_{J}$ of $\mathcal{T}$, we mainly study $\mathrm{Sel}_{J}^{\mathrm{Gr}}$ rather than $\mathrm{Sel}_{J}^{\mathrm{BK}}$ because of the simplicity of the definition of $\operatorname{Sel}_{J}^{\mathrm{Gr}}$. We denote $\mathrm{Sel}_{J}^{\mathrm{Gr}}$ by $\mathrm{Sel}_{J}$ for brevity if this causes no confusion (note that $\mathrm{Sel}_{J}^{\mathrm{Gr}}$ and $\mathrm{Sel}_{J}^{\mathrm{BK}}$ are different in general).

\subsection{Surjectivity of localization maps}

In this subsection, we give the surjectivity of localization maps from semi-global Galois cohomologies to certain local Galois cohomologies at decomposition groups (Theorem 4.10 and Corollary 4.12). The result in this section was used before in $\S \S 4.1-4.3$ and will be used in $\S \S 5,7$ and 8 .

Let $\mathcal{R}$ be a 'deformation ring' and let $M$ be a rank-two Galois representation over $\mathcal{R}$. In this subsection, we will study the following situations:

(1) $\mathcal{R}=\mathbb{H}_{\mathcal{F}}^{\text {n.o }}$ and $M=\mathcal{T}$;

(2) $\mathcal{R}=\mathcal{O}_{\mathfrak{I}}[[\Gamma]]$ and $M=\mathcal{T}_{I}$ where $I=\operatorname{Ker}(\mathfrak{I}) \mathbb{H}_{\mathcal{F}}^{\text {n.o }}$ for $\mathfrak{I} \in \mathfrak{X}_{\text {arith }}\left(\mathbb{H}_{\mathcal{F}}^{\text {ord }}\right)_{\geqslant 0}$ (the one-variable deformation case (a) introduced in $\S 2$ );

(3) $\mathcal{R}=\mathbb{H}_{\mathcal{F}}^{\text {ord }}$ and $M=\mathcal{T}_{(\gamma-\chi(\gamma))}$ or $M=\mathcal{T}_{\left(\gamma-\kappa\left(\gamma^{\prime}\right) \gamma^{\prime}\right)}$ (case (b) or (c) introduced in $\S 2$ ).

Let $\mathcal{R}$ and $M$ be in one of the above three situations and let $H$ be an ideal of $\mathcal{R}$ such that $\mathcal{R} / H$ is finite flat over $\mathbb{Z}_{p}$ (hence, $H$ is of height-two in case (1) and $H$ is of height-one in cases (2) 


\section{T. OCHIAI}

and (3)). Since $M \otimes_{\mathcal{R}} \mathcal{R}^{\vee}[H]$ is of cofinite type over $\mathbb{Z}_{p}$, the subgroups $H_{f}^{1}\left(\mathbb{Q}_{v}, M \otimes_{\mathcal{R}} \mathcal{R}^{\vee}[H]\right)$ of $H^{1}\left(\mathbb{Q}_{v}, M \otimes_{\mathcal{R}} \mathcal{R}^{\vee}[H]\right)$ are defined as in $\S 4.1$. When we have a system of such ideals $S=\{H \subset \mathcal{R}\}$ such that $\lim _{\mathcal{R}} / H=\mathcal{R}$, we would like to discuss whether the natural localization map

$$
H^{1}\left(\mathbb{Q}_{\Sigma} / \mathbb{Q}, M \otimes_{\mathcal{R}} \mathcal{R}^{\vee}\right) \longrightarrow \bigoplus_{v \in \Sigma \backslash\{\infty\}} \underset{\lim }{\longrightarrow} \frac{H^{1}\left(\mathbb{Q}_{v}, M \otimes_{\mathcal{R}} \mathcal{R}^{\vee}[H]\right)}{H_{f}^{1}\left(\mathbb{Q}_{v}, M \otimes_{\mathcal{R}} \mathcal{R}^{\vee}[H]\right)}
$$

is surjective. The following theorem is obtained from a variant of the global duality theorem in our situation.

THEOREM 4.10. Let us consider the following cases.

(A) $\mathcal{R}$ and $M$ are as in case (1) and $H$ runs height-two ideals $\Delta_{s, t}^{(j, k)}$ for $s, t \geqslant 0$ with fixed $j, k$.

(B) $\mathcal{R}$ and $M$ are as in case (2) and $H$ runs height-one ideals $\Phi_{s}^{(j)} \subset \mathcal{O}_{\mathfrak{I}}[[\Gamma]]$ for $s \geqslant 0$ with fixed $j \geqslant 1$.

(C) $\mathcal{R}$ and $M$ are as in case (3) and $H$ runs height-one ideals $\Psi_{t}^{(k)} \subset \mathbb{H}_{\mathcal{F}}^{\text {ord }}$ for $t \geqslant 0$ with fixed $k \geqslant 2$.

The localization map

$$
H^{1}\left(\mathbb{Q}_{\Sigma} / \mathbb{Q}, M \otimes_{\mathcal{R}} \mathcal{R}^{\vee}\right) \longrightarrow \bigoplus_{v \in \Sigma \backslash\{\infty\}} \frac{\lim }{H} \frac{H^{1}\left(\mathbb{Q}_{v}, M \otimes_{\mathcal{R}} \mathcal{R}^{\vee}[H]\right)}{H_{f}^{1}\left(\mathbb{Q}_{v}, M \otimes_{\mathcal{R}} \mathcal{R}^{\vee}[H]\right)}
$$

is surjective in each of cases $(A),(B)$ or $(C)$.

Proof. By the global duality theorem, we have the following exact sequence

$$
\begin{aligned}
0 \longrightarrow \underset{H, n}{\lim _{H}} \operatorname{Sel}_{M /\left(H, p^{p}\right) M}^{\mathrm{BK}} \longrightarrow \underset{H}{\lim } H^{1}\left(\mathbb{Q}_{\Sigma} / \mathbb{Q}, M \otimes_{\mathcal{R}} \mathcal{R}^{\vee}[H]\right) \\
\longrightarrow \bigoplus_{v \in \Sigma \backslash\{\infty\}} \underset{H, n}{\lim } \frac{H^{1}\left(\mathbb{Q}_{v}, M \otimes_{\mathcal{R}} \mathcal{R}^{\vee}[H]\right)}{H_{f}^{1}\left(\mathbb{Q}_{v}, M \otimes_{\mathcal{R}} \mathcal{R}^{\vee}[H]\right)} \longrightarrow\left(\stackrel{\operatorname{limSel}}{H, n}_{M^{\vee}(1)\left[H, p^{n}\right]}^{\mathrm{BK}}\right)^{\vee},
\end{aligned}
$$

where $\operatorname{Sel}_{M^{\vee}(1)\left[H, p^{n}\right]}^{\mathrm{BK}}$ is defined as

$$
\operatorname{Sel}_{M^{\vee}(1)\left[H, p^{n}\right]}^{\mathrm{BK}}=\operatorname{Ker}\left[H^{1}\left(\mathbb{Q}_{\Sigma} / \mathbb{Q}, M^{\vee}(1)\left[H, p^{n}\right]\right) \longrightarrow \bigoplus_{v \in \Sigma \backslash\{\infty\}} \frac{H^{1}\left(\mathbb{Q}_{v}, M^{\vee}(1)\left[H, p^{n}\right]\right)}{H_{f}^{1}\left(\mathbb{Q}_{v}, M^{\vee}(1)\left[H, p^{n}\right]\right)}\right] .
$$

Note that the local condition $H_{f}^{1}\left(\mathbb{Q}_{v}, M^{\vee}(1)\left[H, p^{n}\right]\right) \subset H^{1}\left(\mathbb{Q}_{v}, M^{\vee}(1)\left[H, p^{n}\right]\right)$ for a finite Galois module $M^{\vee}(1)\left[H, p^{n}\right]$ is defined to be the pull-back of $H_{f}^{1}\left(\mathbb{Q}_{v}, M^{\vee}(1)[H]\right) \subset H^{1}\left(\mathbb{Q}_{v}, M^{\vee}(1)[H]\right)$ via the natural map $H^{1}\left(\mathbb{Q}_{v}, M^{\vee}(1)\left[H, p^{n}\right]\right) \longrightarrow H^{1}\left(\mathbb{Q}_{v}, M^{\vee}(1)[H]\right)$.

Since we assume Condition (Ir), $\mathcal{R}$ is a Gorenstein algebra in each case (1), (2) or (3). Hence, we have an involution $\iota: \mathcal{R} \longrightarrow \mathcal{R}$, which coincides with the canonical involution $g \mapsto g^{-1}$ of $\mathbb{Z}_{p}\left[\left[\Gamma \times \Gamma^{\prime}\right]\right] \subset \mathcal{R}$ (respectively $\mathbb{Z}_{p}[[\Gamma]] \subset \mathcal{R}, \mathbb{Z}_{p}\left[\left[\Gamma^{\prime}\right]\right] \subset \mathcal{R}$ ) for $g \in \Gamma \times \Gamma^{\prime}$ (respectively $g \in \Gamma$, $g \in \Gamma^{\prime}$ ) in case (1) (respectively (2), (3)). Let us denote by $M^{\iota}$ a free $\mathcal{R}$-module of rank two $\lim _{H, n} M^{\vee}(1)\left[H, p^{n}\right]$. By Condition (Ir), the natural restriction map $\operatorname{Sel}_{M^{\vee}(1)\left[H, p^{n}\right]}^{\mathrm{BK}} \longrightarrow \operatorname{Sel}_{M^{\iota}}^{\mathrm{BK}}\left[H, p^{n}\right]$ is injective, where $\operatorname{Sel}_{M^{\iota}}^{\mathrm{BK}}=\lim _{H, n} \operatorname{Sel}_{M^{\iota} /\left(H, p^{n}\right) M^{\iota}}^{\mathrm{BK}}$. Thus, it suffices to show that $\varliminf_{H, n} \operatorname{Sel}_{M^{\iota}}^{\mathrm{BK}}\left[H, p^{n}\right]$ is zero in order to have the desired surjectivity.

We refer the reader to $[\mathrm{Och} 03, \S 5]$ for the above facts and the following lemma.

Lemma 4.11. We have the following $\mathcal{R}$-linear isomorphism:

$$
\operatorname{Sel}_{M^{\iota}}^{\mathrm{BK}}\left[H, p^{n}\right] \cong \operatorname{Hom}_{\mathcal{R} /\left(H, p^{n}\right)}\left(\left(\operatorname{Sel}_{M^{\iota}}^{\mathrm{BK}}\right)^{\vee} /\left(H, p^{n}\right)\left(\operatorname{Sel}_{M^{\iota}}^{\mathrm{BK}}\right)^{\vee}, \mathcal{R} /\left(H, p^{n}\right)\right)^{\iota}
$$

where $(\cdot)^{\iota}$ means the twist of an $\mathcal{R}$-module structure via the involution $\iota$. 


\section{ON THE TWO-VARIABLE IWASAWA MAIN CONJECTURE}

By taking the projective limit of this isomorphism, we have:

$$
\lim _{H, n} \operatorname{Sel}_{M^{\vee}(1)\left[H, p^{n}\right]}^{\mathrm{BK}} \cong \operatorname{Hom}_{\mathcal{R}}\left(\left(\operatorname{Sel}_{M^{\iota}}^{\mathrm{BK}}\right)^{\vee}, \mathcal{R}\right)
$$

Since $\left(\mathrm{Sel}_{M^{\iota}}^{\mathrm{BK}}\right)^{\vee}$ is a torsion $\mathcal{R}$-module, by Proposition 4.9(2) and its proof, the proof is completed.

Corollary 4.12. Let $\mathcal{R}$ and $M$ be one of the pairs given in this subsection. Then the localization map

$$
H^{1}\left(\mathbb{Q}_{\Sigma} / \mathbb{Q}, M \otimes_{\mathcal{R}} \mathcal{R}^{\vee}\right) \longrightarrow \frac{H^{1}\left(\mathbb{Q}_{p}, M \otimes_{\mathcal{R}} \mathcal{R}^{\vee}\right)}{H_{\mathrm{Gr}}^{1}\left(\mathbb{Q}_{p}, M \otimes_{\mathcal{R}} \mathcal{R}^{\vee}\right)} \oplus \underset{v \in \Sigma \backslash\{p, \infty\}}{\bigoplus} \frac{H^{1}\left(\mathbb{Q}_{v}, M \otimes_{\mathcal{R}} \mathcal{R}^{\vee}\right)}{H_{\mathrm{ur}}^{1}\left(\mathbb{Q}_{v}, M \otimes_{\mathcal{R}} \mathcal{R}^{\vee}\right)}
$$

is surjective.

Proof. The corollary is a consequence of Theorem 4.10 because $\lim _{H} H_{f}^{1}\left(\mathbb{Q}_{v}, M \otimes_{\mathcal{R}} \mathcal{R}^{\vee}[H]\right)$ is contained in $H_{\mathrm{Gr}}^{1}\left(\mathbb{Q}_{p}, M \otimes_{\mathcal{R}} \mathcal{R}^{\vee}\right)$ (respectively $H_{\mathrm{ur}}^{1}\left(\mathbb{Q}_{v}, M \otimes_{\mathcal{R}} \mathcal{R}^{\vee}\right)$ ) when $v=p$ (respectively $v \in \Sigma \backslash\{p, \infty\})$.

\section{Control theorem for Greenberg's Selmer groups}

For a Galois representation $M \cong \mathcal{R}^{d}$ of $G_{\mathbb{Q}}$ and a prime ideal $J$ of $\mathcal{R}$, we have the natural restriction map between Selmer groups $\operatorname{Sel}_{M / J M} \stackrel{\text { res }_{J}}{\longrightarrow} \operatorname{Sel}_{M}[J]$ (if they are defined). What we call the control theorem is the type of problems (or theorems) where we study the kernel and the cokernel of res $J$ (or equivalently its Pontryagin dual $\left.\left(\operatorname{Sel}_{M}\right)^{\vee} / J\left(\operatorname{Sel}_{M}\right)^{\vee} \longrightarrow\left(\operatorname{Sel}_{M / J M}\right)^{\vee}\right)$. For a family $M$ over a one-variable algebra $\mathcal{R}$ and its specialization to a zero-variable algebra (i.e. a discrete valuation ring) $\mathcal{R} / J$, the control theorem has already been studied in [Och01].

In this section, we study the control theorem for a nearly ordinary deformation $\mathcal{T}$ or quotient representations of $\mathcal{T}$. Throughout the section, we denote by $N_{J}$ the quotient $N / J N$ for an $\mathbb{H}_{\mathcal{F}}^{\text {n.o }}-$ module $N$ and an ideal $J$ of $\mathbb{H}_{\mathcal{F}}^{\text {n.o }}$ for short. We will always assume Condition (Ir) throughout the section. The assertions on Coker $\left(\operatorname{res}_{J}\right)$ hold without Condition (Ir). However, the assertion on $\operatorname{Ker}\left(\operatorname{res}_{J}\right)$ might be modified if we replace Condition (Ir) with a weaker condition. Although it is not difficult, we decided not to do this in order to avoid an unnecessarily complicated description. We refer the reader to [Och01] for the idea of such an argument in the case without Condition (Ir).

\subsection{From two variables to one variable}

First, we discuss the specialization of the two-variable $\mathrm{Sel}_{\mathcal{T}}$ to some of the important one-variable deformations.

Proposition 5.1. Assume Condition (Ir) for $\mathcal{T}=\mathcal{T}_{\mathcal{F}}^{(i)}$. Let $J$ be a height-one prime ideal of $\mathbb{H}_{\mathcal{F}}^{\text {noo }}$ and let res $J$ be the restriction map $\operatorname{Sel}_{J} \longrightarrow \operatorname{Sel}_{\mathcal{T}}[J]$, where $\operatorname{Sel}_{J}=\operatorname{Sel}_{\mathcal{T} / J \mathcal{T}}$. Then the map res $J$ is injective. Coker $\left(\operatorname{res}_{J}\right)$ is a sub-quotient of the following group $\mathcal{L}_{J}$ :

$$
\mathcal{L}_{J}= \begin{cases}\left(\mathrm{F}^{-} \mathcal{A}\left[\gamma-\kappa\left(\gamma^{\prime}\right) \gamma^{\prime}\right] J\right)^{G_{\mathbb{Q}_{p}}} \oplus \bigoplus_{v \in \Sigma \backslash\{p, \infty\}}\left(\left(\mathcal{A}^{I_{v}}\right)_{J}\right)^{G_{\mathbb{Q}_{v}}} & \text { if } \left.\mathrm{F}^{-} \mathcal{A}[\mathfrak{M}]\right]^{I_{p}} \neq 0 \\ \bigoplus_{v \in \Sigma \backslash\{p, \infty\}}\left(\left(\mathcal{A}^{I_{v}}\right)_{J}\right)^{G_{\mathbb{Q}_{v}}} & \text { if } \mathrm{F}^{-} \mathcal{A}[\mathfrak{M}]^{I_{p}}=0\end{cases}
$$

where $M_{J}$ means $M / J M$ for an $\mathbb{H}_{\mathcal{F}}^{\text {n.o }}$-module $M$. Further, if we have the surjectivity of the localization map

$$
H^{1}\left(\mathbb{Q}_{\Sigma} / \mathbb{Q}, \mathcal{A}[J]\right) \stackrel{\operatorname{loc}_{J}}{\longrightarrow} \frac{H^{1}\left(\mathbb{Q}_{p}, \mathcal{A}[J]\right)}{H_{\mathrm{Gr}}^{1}\left(\mathbb{Q}_{p}, \mathcal{A}[J]\right)} \oplus \underset{v \in \Sigma \backslash\{p, \infty\}}{\bigoplus} \frac{H^{1}\left(\mathbb{Q}_{v}, \mathcal{A}[J]\right)}{H_{\mathrm{ur}}^{1}\left(\mathbb{Q}_{v}, \mathcal{A}[J]\right)}
$$

then the cokernel of res $J$ is isomorphic to $\mathcal{L}_{J}$. 


\section{T. OCHIAI}

Proof. Let us recall a diagram as follows:

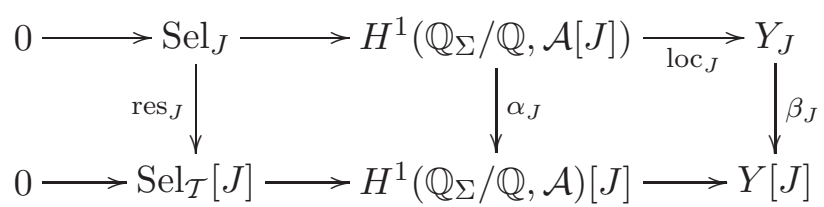

where

$$
\begin{aligned}
Y_{J} & =\frac{H^{1}\left(\mathbb{Q}_{p}, \mathcal{A}[J]\right)}{H_{\mathrm{Gr}}^{1}\left(\mathbb{Q}_{p}, \mathcal{A}[J]\right)} \oplus \bigoplus_{v \in \Sigma \backslash\{p, \infty\}} \frac{H^{1}\left(\mathbb{Q}_{v}, \mathcal{A}[J]\right)}{H_{\mathrm{ur}}^{1}\left(\mathbb{Q}_{v}, \mathcal{A}[J]\right)} \\
Y & =\frac{H^{1}\left(\mathbb{Q}_{p}, \mathcal{A}\right)}{H_{\mathrm{Gr}}^{1}\left(\mathbb{Q}_{p}, \mathcal{A}\right)} \oplus \bigoplus_{v \in \Sigma \backslash\{p, \infty\}} \frac{H^{1}\left(\mathbb{Q}_{v}, \mathcal{A}\right)}{H_{\mathrm{ur}}^{1}\left(\mathbb{Q}_{v}, \mathcal{A}\right)} .
\end{aligned}
$$

By Condition (Ir), the map $\alpha_{J}$ is injective. Consequently, res ${ }_{J}$ is injective. By the snake lemma and by the injectivity of $\alpha_{J}$, Coker(res $\operatorname{res}_{J}$ ) is isomorphic to a submodule of $\operatorname{Ker}\left(\beta_{J}\right)$. Further, we have $\operatorname{Coker}\left(\operatorname{res}_{J}\right) \cong \operatorname{Ker}\left(\beta_{J}\right)$ if $\operatorname{loc}_{J}$ is surjective. Hence, we only have to show that $\operatorname{Ker}\left(\beta_{J}\right)$ is isomorphic to $\mathcal{L}_{J}$. By the inflation-restriction sequence, it is easy to see that the kernel of

$$
\frac{H^{1}\left(\mathbb{Q}_{v}, \mathcal{A}[J]\right)}{H_{\mathrm{ur}}^{1}\left(\mathbb{Q}_{v}, \mathcal{A}[J]\right)} \longrightarrow \frac{H^{1}\left(\mathbb{Q}_{v}, \mathcal{A}\right)}{H_{\mathrm{ur}}^{1}\left(\mathbb{Q}_{v}, \mathcal{A}\right)}
$$

is $\left(\left(\mathcal{A}^{I_{v}}\right)_{J}\right)^{G_{\mathbb{Q} v}}$ at each $v \in \Sigma \backslash\{p, \infty\}$. In the rest of the proof, we will concentrate on the map $\beta_{J}$ restricted to the $p$-part. Let us consider the exact sequence:

$$
0 \longrightarrow H_{\mathrm{Gr}}^{1}\left(\mathbb{Q}_{p}, \mathcal{A}[J]\right) \longrightarrow H^{1}\left(\mathbb{Q}_{p}, \mathcal{A}[J]\right) \longrightarrow H^{1}\left(I_{p}, \mathrm{~F}^{-} \mathcal{A}[J]\right)^{G_{\mathbb{Q}_{p}}}
$$

Note that the second map $H^{1}\left(\mathbb{Q}_{p}, \mathcal{A}[J]\right) \longrightarrow H^{1}\left(I_{p}, \mathrm{~F}^{-} \mathcal{A}[J]\right)^{G_{\mathbb{Q}_{p}}}$ decomposes as

$$
H^{1}\left(\mathbb{Q}_{p}, \mathcal{A}[J]\right) \stackrel{a}{\longrightarrow} H^{1}\left(\mathbb{Q}_{p}, \mathrm{~F}^{-} \mathcal{A}[J]\right) \stackrel{b}{\longrightarrow} H^{1}\left(I_{p}, \mathrm{~F}^{-} \mathcal{A}[J]\right)^{G_{\mathbb{Q}_{p}}}
$$

The map $a$ is surjective as is shown in Claim 4.8 and the map $b$ is surjective since the cohomological dimension of $G_{\mathbb{Q}_{p}} / I_{p}$ is one. Thus, we have

$$
\frac{H^{1}\left(\mathbb{Q}_{p}, \mathcal{A}[J]\right)}{H_{\mathrm{Gr}}^{1}\left(\mathbb{Q}_{p}, \mathcal{A}[J]\right)} \cong H^{1}\left(I_{p}, \mathrm{~F}^{-} \mathcal{A}[J]\right)^{G_{\mathbb{Q}_{p}}}
$$

By a similar argument, we have

$$
\frac{H^{1}\left(\mathbb{Q}_{p}, \mathcal{A}\right)}{H_{\mathrm{Gr}}^{1}\left(\mathbb{Q}_{p}, \mathcal{A}\right)} \cong H^{1}\left(I_{p}, \mathrm{~F}^{-} \mathcal{A}\right)^{G_{\mathbb{Q}_{p}}}
$$

This gives

$$
\operatorname{Ker}\left[\frac{H^{1}\left(\mathbb{Q}_{p}, \mathcal{A}[J]\right)}{H_{\mathrm{Gr}}^{1}\left(\mathbb{Q}_{p}, \mathcal{A}[J]\right)} \longrightarrow \frac{H^{1}\left(\mathbb{Q}_{p}, \mathcal{A}\right)}{H_{\mathrm{Gr}}^{1}\left(\mathbb{Q}_{p}, \mathcal{A}\right)}\right] \cong\left(\left(\mathrm{F}^{-} \mathcal{A}\right)^{I_{p}} / J\left(\mathrm{~F}^{-} \mathcal{A}\right)^{I_{p}}\right)^{G_{\mathbb{Q}_{p}}}
$$

We complete the proof since

$$
\left(\mathrm{F}^{-} \mathcal{A}\right)^{I_{p}} \cong \begin{cases}\mathrm{F}^{-} \mathcal{A}\left[\gamma-\kappa\left(\gamma^{\prime}\right) \gamma^{\prime}\right] & \text { if } \mathrm{F}^{-} \mathcal{A}[\mathfrak{M}]^{I_{p}} \neq 0 \\ 0 & \text { if } \mathrm{F}^{-} \mathcal{A}[\mathfrak{M}]^{I_{p}}=0 .\end{cases}
$$

We will apply Proposition 5.1 to obtain the following.

Proposition 5.2. Assume Condition (Ir) for $\mathcal{T}=\mathcal{T}_{\mathcal{F}}^{(i)}$. Let us consider height-one primes $J \subset \mathbb{H}_{\mathcal{F}}^{\text {n.o }}$ as follows in the following cases:

(a) $J$ is $I=\operatorname{Ker}(\mathfrak{I}) \mathbb{H}_{\mathcal{F}}^{\text {n.o }}$ for $\mathfrak{I} \in \mathfrak{X}_{\text {arith }}\left(\mathbb{H}_{\mathcal{F}}^{\text {ord }}\right)_{\geqslant 0}$;

(b) $J$ is $(\gamma-\chi(\gamma)) \subset \mathbb{H}_{\mathcal{F}}^{\text {n.o }}$; 


\section{ON THE TWO-VARIABLE IWASAWA MAIN CONJECTURE}

(c) $J$ is $\left(\gamma-\kappa\left(\gamma^{\prime}\right) \gamma^{\prime}\right) \subset \mathbb{H}_{\mathcal{F}}^{\text {n.o }}$;

(d) $J$ is $\left(\gamma^{2}-\kappa^{2}\left(\gamma^{\prime}\right) \gamma^{\prime}\right) \subset \mathbb{H}_{\mathcal{F}}^{\text {n.o }}$.

Then, the restriction map $\operatorname{res}_{J}: \operatorname{Sel}_{J} \longrightarrow \operatorname{Sel}_{\mathcal{T}}[J]$ is injective in every case and we have:

$$
\begin{cases}\operatorname{Coker}\left(\operatorname{res}_{J}\right) \cong\left(U_{\mathfrak{I}}\right)^{\vee} & \text { in case (a) with } J=\operatorname{Ker}(\mathfrak{I}), \\ \operatorname{Coker}\left(\operatorname{res}_{J}\right) \cong\left(\mathbb{H}_{\mathcal{F}}\right)^{\vee}{ }^{\vee}\left[A_{p}(\mathcal{F})-1\right] & \text { in case }(c) \text { with } \mathrm{F}^{-} \mathcal{A}[\mathfrak{M}]^{I_{p}} \neq 0 \\ \operatorname{Coker}\left(\operatorname{res}_{J}\right)=0 & \text { otherwise, }\end{cases}
$$

where

$$
U_{\mathfrak{I}}=\bigoplus_{v \in \Sigma \backslash\{p, \infty\}}\left(\left(\left(\mathbb{T}_{\mathcal{F}}^{\text {ord }}\right)^{*}\right)_{I_{v}}[\operatorname{Ker}(\mathfrak{I})] \otimes_{\mathbb{Z}_{p}} \mathbb{Z}_{p}[[\Gamma]]\left(\tilde{\chi}^{-1}\right) \otimes \omega^{-i}\right)^{G_{\mathbb{Q}_{p}}}
$$

Remark 5.3. For each $v \in \Sigma \backslash\{p, \infty\},\left(\left(\mathbb{T}_{\mathcal{F}}^{\text {ord }}\right)^{*}\right)_{I_{v}}[\operatorname{Ker}(\mathfrak{I})]$ is always finite and is trivial except in certain special cases (cf. Theorem 3.3).

Proof. By Proposition 5.1, res $J$ is injective and we have

$$
\operatorname{Coker}\left(\operatorname{res}_{J}\right)= \begin{cases}\left(\mathrm{F}^{-} \mathcal{A}\left[\gamma-\kappa\left(\gamma^{\prime}\right) \gamma^{\prime}\right]_{J}\right)^{G_{\mathbb{Q}_{p}}} \oplus \underset{v \in \Sigma \backslash\{p, \infty\}}{\bigoplus_{v \in \Sigma \backslash\{p, \infty\}}\left(\left(\mathcal{A}^{I_{v}}\right)_{J}\right)^{G_{\mathbb{Q}_{v}}}} & \text { if } \mathrm{F}^{-} \mathcal{A}[\mathfrak{M}]^{I_{p}} \neq 0, \\ \bigoplus_{J}\left(\left(\mathcal{A}^{I_{v}}\right)_{J}\right)_{\mathbb{Q}_{v}} & \text { if } \mathrm{F}^{-} \mathcal{A}[\mathfrak{M}]^{I_{p}}=0 .\end{cases}
$$

Except in case (c), $\mathrm{F}^{-} \mathcal{A}\left[\gamma-\kappa\left(\gamma^{\prime}\right) \gamma^{\prime}\right]_{J}$ is zero. In case (c), $\mathrm{F}^{-} \mathcal{A}\left[\gamma-\kappa\left(\gamma^{\prime}\right) \gamma^{\prime}\right]_{J}=\mathrm{F}^{-} \mathcal{A}\left[\gamma-\kappa\left(\gamma^{\prime}\right) \gamma^{\prime}\right]$ is a cofree $\mathbb{H}_{\mathcal{F}}^{\text {ord }}$-module of rank one with unramified $G_{\mathbb{Q}_{p}}$-action on which Frob $p$ acts via the multipli-

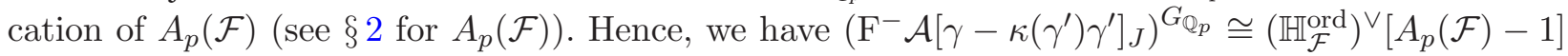
in this case.

Next, we discuss local terms at $v \in \Sigma \backslash\{p, \infty\}$. Recall that

$$
\mathcal{A} \cong\left(\mathbb{T}_{\mathcal{F}}^{\text {ord }} \widehat{\otimes}_{\mathbb{Z}_{p}} \mathbb{Z}_{p}[[\Gamma]](\widetilde{\chi}) \otimes \omega^{i}\right) \otimes_{\mathbb{H}_{\mathcal{F}}^{\text {n.o }}} \operatorname{Hom}_{\mathbb{Z}_{p}}\left(\mathbb{H}_{\mathcal{F}}^{\text {n.o }}, \mathbb{Q}_{p} / \mathbb{Z}_{p}\right)
$$

(see the beginning of $\S 2$ for $\mathbb{T}_{\mathcal{F}}^{\text {ord }}$ ). Since $I_{v}$ acts trivially on $\mathbb{Z}_{p}[[\Gamma]](\widetilde{\chi}) \otimes \omega^{i}$, we have

$$
\left(\left(\left(\mathcal{A}^{I_{v}}\right)_{J}\right)^{G_{\mathbb{Q} v}}\right)^{\vee} \cong\left(\left(\left(\left(\mathbb{T}_{\mathcal{F}}^{\text {ord }}\right)^{*}\right)_{I_{v}} \widehat{\otimes}_{\mathbb{Z}_{p}} \mathbb{Z}_{p}[[\Gamma]]\left(\tilde{\chi}^{-1}\right) \otimes \omega^{-i}\right)[J]\right)_{G_{\mathbb{Q}_{v}}} .
$$

In cases (b), (c) and $(\mathrm{d}),\left(\left(\left(\mathbb{T}_{\mathcal{F}}^{\text {ord }}\right)^{*}\right)_{I_{v}} \widehat{\otimes}_{\mathbb{Z}_{p}} \mathbb{Z}_{p}[[\Gamma]]\left(\tilde{\chi}^{-1}\right) \otimes \omega^{-i}\right)[J]$ is clearly zero. In case (a) for $J=$ $\operatorname{Ker}(\mathfrak{I}) \mathbb{H}_{\mathcal{F}}^{\text {n.o }}$ with certain $\mathfrak{I} \in \mathfrak{X}_{\text {arith }}\left(\mathbb{H}_{\mathcal{F}}^{\text {ord }}\right) \geqslant 0$, we have

$$
\left(\left(\left(\mathbb{T}_{\mathcal{F}}^{\text {ord }}\right)^{*}\right)_{I_{v}} \widehat{\otimes}_{\mathbb{Z}_{p}} \mathbb{Z}_{p}[[\Gamma]]\left(\tilde{\chi}^{-1}\right) \otimes \omega^{-i}\right)[J]=\left(\left(\mathbb{T}_{\mathcal{F}}^{\text {ord }}\right)^{*}\right)_{I_{v}}[\operatorname{Ker}(\mathfrak{I})] \widehat{\otimes}_{\mathbb{Z}_{p}} \mathbb{Z}_{p}[[\Gamma]]\left(\tilde{\chi}^{-1}\right) \otimes \omega^{-i}
$$

This completes the proof.

\subsection{From one variable to the discrete valuation case}

In [Och01], we studied control theorems of the Selmer groups for one-variable Galois deformations when they are specialized into various representations over discrete valuation rings. In this subsection, we restrict ourselves to one-variable deformations inside Hida deformations in order to have more precise and complete results. By applying the fundamental diagram and the snake lemma as in $\S 5.1$, we also prove the control theorem in this case.

Proposition 5.4. Assume condition (Ir) for $\mathcal{T}=\mathcal{T}_{\mathcal{F}}^{(i)}$. Let $J$ and $J^{\prime}$ be two different heightone prime ideals of $\mathbb{H}_{\mathcal{F}}^{\text {n.o }}$ and let res $J_{J^{\prime}}$ be the restriction map $\operatorname{Sel}_{\mathcal{T} /\left(J, J^{\prime}\right) \mathcal{T}^{\prime}} \longrightarrow \operatorname{Sel}_{J}\left[J^{\prime}\right]$, where $\operatorname{Sel}_{J}=\operatorname{Sel}_{\mathcal{T} / J \mathcal{T}}$. Then, the map $\operatorname{res}_{J^{\prime}}$ is injective and Coker(res $\left.J_{J^{\prime}}\right)$ is a sub-quotient of the 


\section{T. OCHIAI}

following group $\mathcal{L}_{J}$ :

$$
\mathcal{L}_{J}= \begin{cases}\left(\left(\mathrm{F}^{-} \mathcal{A}[J]^{I_{p}}\right)_{J^{\prime}}\right)^{G_{\mathbb{Q}_{p}}} \oplus \underset{v \in \Sigma \backslash\{p, \infty\}}{\bigoplus_{v \in \Sigma \backslash\{p, \infty\}}\left(\left(\mathcal{A}[J]^{I_{v}}\right)_{J^{\prime}}\right)^{G_{\mathbb{Q}_{v}}}} & \text { if } \left.\mathrm{F}^{-} \mathcal{A}[\mathfrak{M}]\right]^{I_{p}} \neq 0 \\ \bigoplus_{\mathcal{A}^{\prime}}\left(\left(\mathcal{A}[J]^{I_{v}}\right)_{J^{\prime}}\right)_{\mathbb{Q}_{v}} & \text { if } \left.\mathrm{F}^{-} \mathcal{A}[\mathfrak{M}]\right]^{I_{p}}=0 .\end{cases}
$$

By calculating the term $\mathcal{L}_{J}$ in each case, we have the following proposition.

Proposition 5.5. Assume Condition (Ir) for $\mathcal{T}=\mathcal{T}_{\mathcal{F}}^{(i)}$. We consider the four cases (a), (b), (c) and (d) with the same $J$ as Proposition 5.2. We consider another height-one ideal $J^{\prime}$ :

$$
J^{\prime}= \begin{cases}\left(\gamma-\chi^{j}(\gamma)\right) \text { for a certain } j \text { with } 1 \leqslant j \leqslant w(\mathfrak{I})+1 & \text { in case }(\mathrm{a}), \\ \operatorname{Ker}(\mathfrak{I}) \mathbb{H}_{\mathcal{F}}^{\text {n.o }} \text { for certain } \mathfrak{I} \in \mathfrak{X}_{\text {arith }}\left(\mathbb{H}_{\mathcal{F}}^{\text {ord }}\right)_{\geqslant 0} & \text { in cases }(b),(c) \text { and }(d) .\end{cases}
$$

The kernels and the cokernels of $\operatorname{res}_{J^{\prime}}: \operatorname{Sel}_{\mathcal{T} /\left(J, J^{\prime}\right) \mathcal{T}} \longrightarrow \operatorname{Sel}_{J}\left[J^{\prime}\right]$ are given as follows.

(1) The restriction map res $J^{\prime}$ is injective in each of (a), (b), (c) and (d).

(2) In cases (a), (b), (c) and (d) with $\mathrm{F}^{-} \mathcal{A}[\mathfrak{M}]^{I_{p}}=0$, Coker(res $\left.J^{\prime}\right)$ is a sub-quotient of a finite group $D$ given as follows:

$$
D= \begin{cases}\bigoplus_{v \in \Sigma \backslash\{p, \infty\}}\left(\left(\left(\mathbb{T}_{\mathcal{F}}^{\text {ord }}\right)^{*}\right)_{I_{v}}[\operatorname{Ker}(\mathfrak{I})]\right)^{\vee} & \text { in cases }(b),(c) \text { and }(d), \\ 0 & \text { in case (a). }\end{cases}
$$

In cases (a), (b), (c) and (d) with $\mathrm{F}^{-} \mathcal{A}[\mathfrak{M}]^{I_{p}} \neq 0, \operatorname{Coker}\left(\operatorname{res}_{J^{\prime}}\right)$ is a sub-quotient of the following group:

$$
\begin{cases}\left(\mathcal{O}_{\mathfrak{I}}\right)^{\vee}\left[1-a_{p}\left(f_{\mathfrak{I}}\right)\right] \oplus D & \text { in cases }(\mathrm{a}),(\mathrm{b}) \text { and }(\mathrm{d}), \\ D & \text { in case }(\mathrm{c}) .\end{cases}
$$

Remark 5.6.

(1) Note that $\mathcal{T} /\left(J, J^{\prime}\right) \mathcal{T}$ is isomorphic to $T_{f_{\mathfrak{I}}} \otimes \chi^{j} \omega^{i}$ (respectively $T_{f_{\mathfrak{I}}} \otimes \chi \omega^{i}, T_{f_{\mathfrak{I}}} \otimes \chi^{w(\mathfrak{I})+1} \omega^{i}$, $\left.T_{f_{\mathfrak{I}}} \otimes \chi^{w(\mathfrak{I}) / 2+1} \omega^{i}\right)$ in case (a) (respectively (b), (c), (d)). Hence, in every case, $\operatorname{Sel}_{\mathcal{T} /\left(J, J^{\prime}\right) \mathcal{T}}$ is the Selmer group associated to a twist of a cuspform $f_{\mathfrak{J}}$.

(2) In cases (b), (c) and (d), the group $D$ is a Pontryagin dual of $\left(\mathcal{A}[J]^{I_{v}}\right)_{J^{\prime}}$. Theorem 3.3 immediately implies that $D$ is finite.

(3) The group $\left(\mathcal{O}_{\mathfrak{I}}\right)^{\vee}\left[1-a_{p}\left(f_{\mathfrak{I}}\right)\right]$ is finite when $w(\mathfrak{I}) \neq 0$ by applying Remark $4.5(2)$. Thus, the kernels and the cokernels of $\operatorname{res}_{J^{\prime}}: \operatorname{Sel}_{\mathcal{T} /\left(J, J^{\prime}\right) \mathcal{T}} \longrightarrow \operatorname{Sel}_{J}\left[J^{\prime}\right]$ are finite if we choose $J$ (respectively $J^{\prime}$ ) so that $w(\mathfrak{I}) \neq 0$ in case (a) (respectively in cases (b) and (d)).

(4) The control theorem in case (a) was studied in various references (e.g. [Gre99]) when $f_{\mathfrak{I}}$ is associated to an elliptic curve $E$. Note that there has been a contribution of the local Tamagawa number of $E$ at every $v \in \Sigma \backslash\{p, \infty\}$ to $\operatorname{Coker}\left(\operatorname{res}_{J^{\prime}}\right.$ ) in the above-mentioned references (e.g. [Gre99]), whereas there is no such contribution in our result. This is because $\operatorname{Sel}_{T_{f_{\mathcal{J}}} \otimes \chi \omega}$ is isomorphic to the classical Selmer group for $E$ only after being divided by a finite abelian group whose order is related to the local Tamagawa number of $E$ at $v$.

\section{Two-variable $p$-adic $L$-function}

In this section, we discuss the two-variable $p$-adic $L$-function for a nearly ordinary deformation $\mathcal{T}$ through Beilinson-Kato elements. The construction will be done by using the two-variable Coleman map (Theorem 6.3), which translates a norm compatible elements to a measure. 


\section{ON THE TWO-VARIABLE IWASAWA MAIN CONJECTURE}

The key to this section is an optimization of the two-variable Beilinson-Kato element given in Theorem 6.11. The results of this section make clear the relation between Kitagawa's two-variable $p$-adic $L$-function (see [Kit94]) and our Euler system construction, modifying previous constructions by [Och03] and [Fuk03] which were not well-optimized in general.

\subsection{Review of the work of [Och03]}

In order to introduce Beilinson-Kato elements, we need to prepare notation. For a normalized eigencuspform $f=\sum_{n>0} a_{n}(f) q^{n}$ of weight $k \geqslant 2$, we denote by $\mathbb{Q}_{f}$ a finite extension of $\mathbb{Q}$ obtained by adjoining all Fourier coefficients of $f$ to $\mathbb{Q}$. We denote by $\bar{f}=\sum_{n>0} a_{n}(f)^{\sigma} q^{n}$ the dual modular form of $f$ where $\sigma$ is a complex conjugation. The dual modular form $\bar{f}$ is known to be a Hecke eigen-cuspform of weight $k$ with Neben character dual of that of $f$. The field $\mathbb{Q}_{f}$ is equal to $\mathbb{Q}_{\bar{f}}$. We associate the de Rham realization $V_{\mathrm{dR}}(f)$ to $f$. The de Rham realization $V_{\mathrm{dR}}(f)$ has the following properties.

(1) $V_{\mathrm{dR}}(f)$ is a two-dimensional vector space over $\mathbb{Q}_{f}$ and is equipped with a de Rham filtration $\mathrm{Fil}^{i} V_{\mathrm{dR}}(f) \subset V_{\mathrm{dR}}(f)$, which is a decreasing filtration of $\mathbb{Q}_{f}$-vector spaces.

(2) We have $\mathrm{Fil}^{0} V_{\mathrm{dR}}(f)=V_{\mathrm{dR}}(f)$ and $\mathrm{Fil}^{k} V_{\mathrm{dR}}(f)=\{0\}$. For each $j$ such that $1 \leqslant j \leqslant k-1$, Fil $^{j} V_{\mathrm{dR}}(f)$ is naturally identified with the one-dimensional $\mathbb{Q}_{f}$-vector space $\mathbb{Q}_{f} \cdot f$.

(3) Let $\mathfrak{I}$ be an arithmetic point of weight $w(\mathfrak{I}) \geqslant 0$. For each $j$ such that $1 \leqslant j \leqslant k-1$, $\mathrm{Fil}^{k-j} V_{\mathrm{dR}}\left(\bar{f}_{\mathfrak{I}}\right) \otimes_{\mathbb{Q}_{f_{\mathfrak{I}}}} K_{\mathfrak{I}}$ is naturally identified with Fil ${ }^{0} \mathrm{D}_{\mathrm{dR}}\left(V_{f_{\mathfrak{I}}}^{*} \otimes \chi^{1-j} \omega^{1-i}\right)$, where $K_{\mathfrak{I}}$ is the $p$-adic completion of $\mathbb{Q}_{f_{\mathfrak{I}}}$ with respect to the fixed embedding $\mathbb{Q}_{f_{\mathfrak{I}}} \subset \overline{\mathbb{Q}} \hookrightarrow \overline{\mathbb{Q}}_{p}, V_{f_{\mathfrak{I}}}$ is $T_{f_{\mathfrak{I}}} \otimes_{\mathbb{Z}_{p}} \mathbb{Q}_{p}$ and ${ }^{*}$ means the $\mathbb{Q}_{p}$-linear dual here. Recall that $D_{\mathrm{dR}}$ is the de Rham functor defined by Fontaine, which is a functor from the category of $p$-adic representations of $G_{\mathbb{Q}_{p}}$ to the category of filtered modules over $\mathbb{Q}_{p}$ (cf. [Fon94]).

For each $1 \leqslant j \leqslant w(\mathfrak{I})+1$, we denote by $\bar{\delta}_{\mathfrak{I}}^{\mathrm{dR}}$ the $\mathbb{Q}_{f_{\mathfrak{I}}}$-basis of Fil ${ }^{w(\mathfrak{I})+2-j} V_{\mathrm{dR}}\left(\bar{f}_{\mathfrak{I}}\right)$ sent to $\bar{f}_{\mathfrak{I}}$ under the natural identification Fil ${ }^{w(\mathfrak{I})+2-j} V_{\mathrm{dR}}\left(\bar{f}_{\mathfrak{I}}\right)=\mathbb{Q}_{f_{\mathfrak{I}}} \cdot \bar{f}_{\mathfrak{I}}$. Let $\mathcal{D}$ be an $\mathbb{H}_{\mathcal{F}}^{\text {ord }}$-module $\left(\mathbb{H}_{\mathcal{F}}^{\text {ord }}(\widetilde{\alpha}) \widehat{\otimes}_{\mathbb{Z}_{p}} \widehat{\mathbb{Z}}_{p}^{\text {ur }}\right)^{G_{\mathbb{Q}_{p}}}$ and let $\delta_{\mathbb{Q}_{p}(1)}$ be the inverse image of $1 \in \mathbb{Q}_{p}$ via the isomorphism $D_{\mathrm{dR}}\left(\mathbb{Q}_{p}(1)\right) \stackrel{\sim}{\longrightarrow} \mathbb{Q}_{p}$ determined by a fixed norm compatible system $\left\{\zeta_{p^{n}}\right\}_{n \geqslant 1}$ of $p^{n}$ th roots of unity. We recall the following properties (see $[\mathrm{Och} 03, \S 3]$ for the proof).

Lemma 6.1. We have the following properties.

(i) $\mathcal{D}$ is a free $\mathbb{H}_{\mathcal{F}}^{\text {ord }}$-module of rank one.

(ii) $\mathcal{D} / \operatorname{Ker}(\mathfrak{I}) \mathcal{D}$ is the canonical lattice of $\mathrm{D}_{\mathrm{dR}}\left(\mathrm{F}^{+} V_{f_{\mathfrak{I}}}\right)=\mathrm{D}_{\text {crys }}\left(\mathrm{F}^{+} V_{f_{\mathfrak{I}}}\right)$ for each $\mathfrak{I} \in \mathfrak{X}_{\text {arith }}\left(\mathbb{H}_{\mathcal{F}}^{\text {ord }}\right) \geqslant 0$.

(iii) For each $(j, \mathfrak{I})$ such that $1 \leqslant j \leqslant w(\mathfrak{I})+1$, we have the canonical isomorphism $\mathrm{D}_{\mathrm{dR}}\left(\mathrm{F}^{+} V_{f_{\mathfrak{I}}} \otimes\right.$ $\left.\chi^{j} \omega^{i}\right) \cong \mathrm{D}_{\mathrm{dR}}\left(V_{f_{\mathfrak{I}}} \otimes \chi^{j} \omega^{i}\right) / \mathrm{Fil}^{0} \mathrm{D}_{\mathrm{dR}}\left(V_{f_{\mathfrak{I}}} \otimes \chi^{j} \omega^{i}\right)$.

(iv) The fixed norm compatible system $\left\{\zeta_{p^{n}}\right\}_{n \geqslant 1}$ induces the following isomorphism:

$$
\mathrm{D}_{\mathrm{dR}}\left(\mathrm{F}^{+} V_{f_{\mathfrak{I}}}\right) \underset{\otimes \delta_{\mathbb{Q}_{p}(1)}^{\otimes j}}{\sim} \mathrm{D}_{\mathrm{dR}}\left(\mathrm{F}^{+} V_{f_{\mathfrak{I}}} \otimes \chi^{j} \omega^{j}\right) \cong \mathrm{D}_{\mathrm{dR}}\left(\mathrm{F}^{+} V_{f_{\mathfrak{I}}} \otimes \chi^{j} \omega^{i}\right)
$$

where $0 \leqslant i \leqslant p-2$.

Definition 6.2. Fix an $\mathbb{H}_{\mathcal{F}}^{\text {ord }}$-basis $d$ of $\mathcal{D}$. For each $\mathfrak{I} \in \mathfrak{X}_{\text {arith }}\left(\mathbb{H}_{\mathcal{F}}^{\text {ord }}\right)_{\geqslant 0}$, we define a de Rham $p$-adic error term $C_{p, \Im, d} \in \overline{\mathbb{Q}}_{p}$ to be

$$
C_{p, \mathfrak{I}, d}=\left\langle\bar{\delta}_{\mathfrak{I}}^{\mathrm{dR}}, d_{\mathfrak{I}} \otimes \delta_{\mathbb{Q}_{p}(1)}^{\otimes j}\right\rangle_{\mathrm{dR}, p}
$$

where $j$ is an integer satisfying $1 \leqslant j \leqslant w(\mathfrak{I})+1,\langle\cdot, \cdot\rangle_{\mathrm{dR}, p}$ is the pairing

$$
\langle\cdot, \cdot\rangle_{\mathrm{dR}, p}: \mathrm{Fil}^{0} \mathrm{D}_{\mathrm{dR}}\left(V_{f_{\mathfrak{I}}}^{*} \otimes \chi^{1-j} \omega^{1-i}\right) \times \mathrm{D}_{\mathrm{dR}}\left(\mathrm{F}^{+} V_{f_{\mathfrak{I}}} \otimes \chi^{j} \omega^{i}\right) \longrightarrow \mathrm{D}_{\mathrm{dR}}\left(\mathbb{Q}_{p}(1)\right) \otimes K_{\mathfrak{I}} \cong K_{\mathfrak{I}}
$$




\section{T. OCHIAI}

induced by the identification of Lemma 6.1(3) and the de Rham pairing:

$$
\mathrm{Fil}^{0} \mathrm{D}_{\mathrm{dR}}\left(V_{f_{\mathfrak{I}}}^{*} \otimes \chi^{1-j} \omega^{1-i}\right) \times \mathrm{D}_{\mathrm{dR}}\left(V_{f_{\mathfrak{I}}} \otimes \chi^{j} \omega^{i}\right) / \mathrm{Fil}^{0} \mathrm{D}_{\mathrm{dR}}\left(V_{f_{\mathfrak{I}}} \otimes \chi^{j} \omega^{i}\right) \longrightarrow \mathrm{D}_{\mathrm{dR}}\left(\mathbb{Q}_{p}(1)\right) \otimes K_{\mathfrak{I}},
$$

and $d_{\mathfrak{I}} \in \mathrm{D}_{\mathrm{dR}}\left(\mathrm{F}^{+} V_{f_{\mathfrak{I}}}\right)$ is the specialization modulo $\operatorname{Ker}(\mathfrak{I})$ of $d \in \mathcal{D}$ (cf. Lemma 6.1(2)).

The $p$-adic error term $C_{p, \mathfrak{I}, d}$ does not depend on $j$ and depends only on $d$ and a fixed norm compatible system $\left\{\zeta_{p^{n}}\right\}_{n \geqslant 1}$ of $p^{n}$ th roots of unity.

For a free $\mathbb{Z}_{p}$-module with continuous $G_{\mathbb{Q}_{p}}$-action $T$, we denote by $H_{/ f}^{1}\left(\mathbb{Q}_{p}, T\right)$ the quotient module $H^{1}\left(\mathbb{Q}_{p}, T\right) / H_{f}^{1}\left(\mathbb{Q}_{p}, T\right)$. We have the dual exponential map exp* $: H_{/ f}^{1}\left(\mathbb{Q}_{p}, T\right) \longrightarrow \mathrm{Fil}^{0} \mathrm{D}_{\mathrm{dR}}(V)$ introduced by Kato [Kat93] where $V=T \otimes_{\mathbb{Z}_{p}} \mathbb{Q}_{p}$. In [Och03], we introduced a certain quotient $H_{/ f}^{1}\left(\mathbb{Q}_{p}, \mathcal{T}^{*}(1)\right)$ of $H^{1}\left(\mathbb{Q}_{p}, \mathcal{T}^{*}(1)\right)$ for the two-variable Hida deformation $\mathcal{T}$. We do not recall the definition here since it is not essential for later explanation. We only remark that $H_{/ f}^{1}\left(\mathbb{Q}_{p}, \mathcal{T}^{*}(1)\right)$ interpolates $H_{/ f}^{1}\left(\mathbb{Q}_{p}, T_{f_{\mathfrak{J}}}^{*}(1) \otimes\left(\omega^{i} \eta \chi^{j}\right)^{-1}\right)$ when $\mathfrak{I} \in \mathfrak{X}_{\text {arith }}\left(\mathbb{H}_{\mathcal{F}}^{\text {ord }}\right), \eta$ and $j$ vary. We recall our result on the interpolation of the dual exponential maps as follows.

Theorem 6.3 [Och03, Theorem 3.13]. Let $i$ be an integer such that $0 \leqslant i \leqslant p-2$. We assume Condition (Ir) for a nearly ordinary deformation $\mathcal{T}=\mathcal{T}_{\mathcal{F}}^{(i)}$. Assume, further, that $\mathbb{H}_{\mathcal{F}}$.o is integrally closed in its fraction field $\operatorname{Frac}\left(\mathbb{H}_{\mathcal{F}}^{\text {n.o }}\right)$. Fix an $\mathbb{H}_{\mathcal{F}}^{\text {ord }}$-basis $d$ of $\mathcal{D}=\left(\mathbb{H}_{\mathcal{F}}^{\text {ord }}(\widetilde{\alpha}) \widehat{\otimes}_{\mathbb{Z}_{p}} \widehat{\mathbb{Z}}_{p}^{\text {ur }}\right) G_{\mathbb{Q}_{p}}$ (Lemma 6.1(1)). Then we have a map $\Xi_{d}: H_{/ f}^{1}\left(\mathbb{Q}_{p}, \mathcal{T}^{*}(1)\right) \longrightarrow \mathbb{H}_{\mathcal{F}}^{\text {n.o }}$ which has the following properties.

(i) The map $\Xi_{d}$ is an $\mathbb{H}_{\mathcal{F}}^{\text {n.o }}$-linear pseudo-isomorphism.

(ii) Let $\mathcal{C} \in H_{/ f}^{1}\left(\mathbb{Q}_{p}, \mathcal{T}^{*}(1)\right)$. For each $(j, \mathfrak{I})$ such that $1 \leqslant j \leqslant w(\mathfrak{I})+1$ and for each finite order character $\eta$ of $\Gamma,\left(\chi^{j} \eta \circ \mathfrak{I}\right)\left(\Xi_{d}(\mathcal{C})\right)$ is equal to

$$
\begin{aligned}
\left(1-\frac{\left(\omega^{i-j} \eta\right)(p) p^{j-1}}{a_{p}\left(f_{\mathfrak{I}}\right)}\right)(1 & \left.-\frac{\left(\omega^{i-j} \eta^{-1}\right)(p) a_{p}\left(f_{\mathfrak{I}}\right)}{p^{j}}\right)^{-1} \\
& \times\left(\frac{p^{j-1}}{a_{p}\left(f_{\mathfrak{I}}\right)}\right)^{q(i, j, \eta)} G\left(\omega^{j-i} \eta\right)\left\langle\exp ^{*}\left(\left(\chi^{j} \eta \circ \mathfrak{I}\right)(\mathcal{C})\right), d_{\mathfrak{I}} \otimes \delta_{\mathbb{Q}_{p}(1)}^{\otimes j}\right\rangle_{\mathrm{dR}, p},
\end{aligned}
$$

where $\left(\chi^{j} \eta \circ \mathfrak{I}\right)(\mathcal{C}) \in H_{/ f}^{1}\left(\mathbb{Q}_{p}, T_{f_{\mathfrak{I}}}^{*}(1) \otimes\left(\omega^{i} \eta \chi^{j}\right)^{-1}\right)$ is the specialization of $\mathcal{C}$ via $\chi^{j} \circ \mathfrak{I}, q(i, j, \eta)$ is the p-order of the conductor of $\omega^{j-i} \eta$ and $G\left(\omega^{j-i} \eta\right)$ is the Gauss sum for $\omega^{j-i} \eta$.

\section{$6.2 p$-adic error terms at weight two}

In this subsection, we study the $p$-adic error terms $C_{p, \mathfrak{I}, d}$ in the special cases where $w(\mathfrak{I})=0$. We fix an $\mathbb{H}_{\mathcal{F}}^{\text {ord }}$-basis $d$ of $\mathcal{D}$ throughout this subsection. The main result of this subsection is as follows.

Proposition 6.4. Let $\mathcal{T}=\mathcal{T}_{\mathcal{F}}^{(i)}$ be a nearly ordinary deformation. Then, $C_{p, \mathfrak{I}, d}$ is a $p$-adic unit for every $\mathfrak{I} \in \mathfrak{X}_{\text {arith }}\left(\mathbb{H}_{\mathcal{F}}^{\text {ord }}\right)$ with $w(\mathfrak{I})=0$.

Proof. For an arithmetic point $\mathfrak{I} \in \mathfrak{X}_{\text {arith }}\left(\mathbb{H}_{\mathcal{F}}^{\text {ord }}\right)$ with $w(\mathfrak{I})=0$, let $B_{\mathfrak{I}}$ be the abelian variety associated to the normalized eigen-cuspform $f_{\mathfrak{I}}$ of weight two. $B_{\mathfrak{I}}$ is an abelian variety of dimension $g=\left[\mathbb{Q}_{f_{\mathfrak{I}}}: \mathbb{Q}\right]$ over $\mathbb{Q}$ and we have an injection $\mathbb{Q}_{f_{\mathfrak{I}}} \hookrightarrow \operatorname{End}_{\mathbb{Q}}\left(B_{\mathfrak{I}}\right) \otimes \mathbb{Q}$. Since $f_{\mathfrak{I}}$ is ordinary at $p$, there exists an abelian variety $B_{\mathfrak{I}}^{\prime}$ over $\mathbb{Q}_{p}$ with the following properties (see [Wi186, $\left.\S 2.2\right]$ ).

(1) $B_{\mathfrak{I}}^{\prime}$ is isogenious to a subabelian variety of $B_{\mathfrak{I}} \otimes \mathbb{Q}_{p}$ with $d=\operatorname{dim}\left(B_{\mathfrak{I}}^{\prime}\right)=\left[K_{\mathfrak{I}}: \mathbb{Q}_{p}\right]$ over $\mathbb{Q}_{p}$.

(2) $B_{\mathfrak{J}}^{\prime}$ has totally multiplicative reduction or good ordinary reduction over $\mathbb{Q}_{p}$.

(3) $H_{\text {ét }}^{1}\left(B_{\mathfrak{I}}^{\prime} \otimes_{\mathbb{Q}_{p}} \overline{\mathbb{Q}}_{p}, \mathbb{Q}_{p}\right)$ is isomorphic to $V_{f_{\mathfrak{I}}}$ as a $G_{\mathbb{Q}_{p}}$-module.

Let $B_{\mathfrak{I}}^{\prime \text { t }}$ be the dual abelian variety of $B_{\mathfrak{I}}^{\prime}$. We denote by $\mathcal{B}$ the $p$-divisible group over $\mathbb{Q}_{p}$ associated 


\section{ON THE TWO-VARIABLE IWASAWA MAIN CONJECTURE}

to $B_{\mathfrak{I}}^{\prime \text { t }}$ with its connected part $\mathcal{B}^{0}$. We see that

$$
\begin{aligned}
\operatorname{Fil}^{0} \mathrm{D}_{\mathrm{dR}}\left(V_{f_{\mathfrak{I}}}^{*} \otimes \chi^{1-j} \omega^{1-i}\right) & \cong \operatorname{Fil}^{0} \mathrm{D}_{\mathrm{dR}}\left(H_{\text {ét }}^{1}\left(B_{\mathfrak{I}}^{\prime \mathrm{t}} \otimes \mathbb{Q}_{p} \overline{\mathbb{Q}}_{p}, \mathbb{Q}_{p}\right)\right) \\
& \cong \operatorname{Fil}^{1} H_{\mathrm{dR}}^{1}\left(B_{\mathfrak{I}}^{\prime \mathrm{t}}\right) \cong \mathbb{D}\left(\mathcal{B}^{0}\right),
\end{aligned}
$$

where $H_{\mathrm{dR}}^{1}\left(B_{\mathfrak{I}}^{\prime \mathrm{t}}\right)$ means the de Rham cohomology of $B_{\mathfrak{I}}^{\prime \mathrm{t}}$ and $\mathbb{D}\left(\mathcal{B}^{0}\right)$ is the Dieudonee module for $\mathcal{B}^{0}$. Since $f_{\mathfrak{I}}$ is ordinary, we see that

$$
\begin{aligned}
\operatorname{Fil}^{0} \mathrm{D}_{\mathrm{dR}}\left(V_{f_{\mathfrak{I}}}^{*} \otimes \chi^{1-j} \omega^{1-i}\right) & \cong \mathrm{D}_{\mathrm{dR}}\left(\mathrm{F}^{-} V_{f_{\mathfrak{I}}}^{*} \otimes \chi^{1-j} \omega^{1-i}\right) \\
& \cong \mathrm{D}_{\mathrm{dR}}\left(K_{\mathfrak{I}}\left(\alpha^{-1}\right)\right) .
\end{aligned}
$$

By Definition $6.2, C_{p, \mathfrak{I}, d}$ is a $p$-adic unit if and only if the $K_{\mathfrak{I}}$-basis of $\mathbb{D}\left(\mathcal{B}^{0}\right)$ induced by $\bar{\delta}_{\mathfrak{I}}^{\mathrm{dR}}$ gives an integral basis of $\mathrm{D}_{\mathrm{dR}}\left(K_{\mathfrak{I}}\left(\alpha^{-1}\right)\right)=\left(K_{\mathfrak{I}}\left(\alpha^{-1}\right) \otimes_{\mathbb{Q}_{p}} \widehat{\mathbb{Q}}_{p}^{\text {ur }}\right)^{G_{\mathbb{Q}_{p}}}$ with respect to the lattice $\left(\mathcal{O}_{\mathfrak{I}}\left(\alpha^{-1}\right) \otimes_{\mathbb{Z}_{p}}\right.$ $\left.\widehat{\mathbb{Z}}_{p}^{\text {ur }}\right)^{G_{\mathbb{Q}_{p}}}$. This is clear since $\mathcal{B}^{0}$ is of multiplicative type.

\subsection{Beilinson-Kato element}

Let $H_{\mathrm{B}}^{1}\left(Y_{1}(M)_{\mathbb{C}}, \operatorname{Sym}^{k-2}\left(R^{1} p_{*} A\right)\right)$ be a Betti cohomology and let $H_{\mathrm{B}, c}^{1}\left(Y_{1}(M)_{\mathbb{C}}, \operatorname{Sym}^{k-2}\left(R^{1} p_{*} A\right)\right)$ be the Betti cohomology with compact support, where $p: \mathcal{E} \rightarrow Y_{1}(M)$ is the universal elliptic curve over the affine modular curve $Y_{1}(M)$ and $A$ is a submodule of $\mathbb{C}$. To each normalized eigen-newform $f \in S_{k}\left(\Gamma_{1}(M)\right)$ of weight $k \geqslant 2$, we associate the Betti realization $V_{\mathrm{B}}(f)$. The realization $V_{\mathrm{B}}(f)$ is defined as $H_{\mathrm{B}}^{1}\left(Y_{1}(M)_{\mathbb{C}}, \operatorname{Sym}^{k-2}\left(R^{1} p_{*} \mathbb{Q}_{f}\right)\right)\left[I_{f}\right]\left(\right.$ respectively $\left.H_{\mathrm{B}, c}^{1}\left(Y_{1}(M)_{\mathbb{C}}, \operatorname{Sym}^{k-2}\left(R^{1} p_{*} \mathbb{Q}_{f}\right)\right)\left[I_{f}\right]\right)$, where $I_{f}=\cap\left(T_{l}-a_{l}(f)\right)$ when $T_{l}$ runs the Hecke operators $T_{l} \in \operatorname{End}_{\mathbb{Q}_{f}}\left(S_{k}\left(\Gamma_{1}(M) ; \mathbb{Q}_{f}\right)\right)$ for all primes $l$. The Betti realization $V_{\mathrm{B}}(f)$ has the following properties.

(1) $V_{\mathrm{B}}(f)$ is a two-dimensional vector space over $\mathbb{Q}_{f}$ equipped with natural action of complex conjugate $\sigma$, whose \pm -eigenspace $V_{\mathrm{B}}(f)^{ \pm}$is one-dimensional over $\mathbb{Q}_{f}$.

(2) We have the period maps (cf. [Del79])

$$
\begin{aligned}
& \operatorname{Per}^{+}: \operatorname{Fil}^{j} V_{\mathrm{dR}}(f) \otimes_{\mathbb{Q}_{f}} \mathbb{C} \stackrel{\sim}{\longrightarrow} V_{\mathrm{B}}(f)^{+} \otimes_{\mathbb{Q}_{f}} \mathbb{C} \\
& \operatorname{Per}^{-}:\left(V_{\mathrm{dR}}(f) / \mathrm{Fil}^{j} V_{\mathrm{dR}}(f)\right) \otimes_{\mathbb{Q}_{f}} \mathbb{C} \stackrel{\sim}{\longrightarrow} V_{\mathrm{B}}(f)^{-} \otimes_{\mathbb{Q}_{f}} \mathbb{C}
\end{aligned}
$$

for each $1 \leqslant j \leqslant k-1$.

Let us denote by $\mathcal{H}$ the local system on $Y_{1}(M)_{\mathbb{C}}$ whose fiber $\mathcal{H}_{s}$ at $s \in Y_{1}(M)_{\mathbb{C}}$ is $H_{1}\left(\mathcal{E}_{s}, \mathbb{Z}\right)$. Let $\varphi: \mathfrak{H} \longrightarrow Y_{1}(M)_{\mathbb{C}}$ be the uniformization map. The stalk of $\mathcal{H}$ at $s=\varphi(y i) \in Y_{1}(M)_{\mathbb{C}}$ is identified with $H_{1}(\mathbb{C} /(\mathbb{Z}+\mathbb{Z} y i), \mathbb{Z})=\mathbb{Z}+\mathbb{Z} y i$ for any $y \in(0, \infty)$. We denote by $\beta$ be the element of $\Gamma\left((0, i \infty), \varphi^{-1}(\mathcal{H})\right)$ which corresponds to $1 \in \mathbb{Z}$.

Definition 6.5. Let $f$ be an eigen-cuspform of level $N$ and weight $w+2$.

(i) Let $\delta_{0}^{\mathrm{B}, w}$ be the element of $H_{1}^{\mathrm{B}}\left(Y_{1}(M)_{\mathbb{C}} ;\{\operatorname{cusps}\}, \operatorname{Sym}^{w}(\mathcal{H})\right)$ which represents a path $(0, \infty)$ and $\beta^{w}$. By abuse of notation, we denote by $\delta_{0}^{\mathrm{B}, w}$ the image via the map

$$
H_{1}^{\mathrm{B}}\left(Y_{1}(M)_{\mathbb{C}} ;\{\operatorname{cusps}\}, \operatorname{Sym}^{w}\left(\mathcal{H}_{\mathbb{Q}_{f}}\right)\right) \stackrel{\sim}{\longrightarrow} H_{\mathrm{B}, c}^{1}\left(Y_{1}(M)_{\mathbb{C}}, \operatorname{Sym}^{w}\left(R^{1} p_{*} \mathbb{Q}_{f}\right)\right) \rightarrow V_{\mathrm{B}}(f) .
$$

(ii) Let $V_{\mathrm{B}}(f) \times V_{\mathrm{B}}(f) \stackrel{\langle\cdot \cdot\rangle_{\mathrm{B}}}{\longrightarrow} \mathbb{Q}_{f}$ be the pairing induced from the Poincaré duality:

$$
H_{\mathrm{B}}^{1}\left(Y_{1}(M)_{\mathbb{C}}, \operatorname{Sym}^{w}\left(R^{1} p_{*} \mathbb{Q}_{f}\right)\right) \times H_{\mathrm{B}, c}^{1}\left(Y_{1}(M)_{\mathbb{C}}, \operatorname{Sym}^{w}\left(R^{1} p_{*} \mathbb{Q}_{f}\right)\right) \longrightarrow H_{\mathrm{B}, c}^{2}\left(Y_{1}(M)_{\mathbb{C}}, \mathbb{Q}_{f}\right) \cong \mathbb{Q}_{f} .
$$

Let $\langle\cdot, \cdot\rangle_{\mathrm{B}, \infty}$ be the extension of $\langle\cdot, \cdot\rangle_{\mathrm{B}}$ as follows:

$$
V_{\mathrm{B}}(f) \otimes_{\mathbb{Q}_{f}} \mathbb{C} \times V_{\mathrm{B}}(f) \otimes_{\mathbb{Q}_{f}} \mathbb{C} \stackrel{\langle\cdot \cdot \cdot\rangle_{\mathrm{B}, \infty}}{\longrightarrow} \mathbb{C} .
$$

We have

$$
\left\langle\operatorname{Per}^{+}\left(f_{\mathfrak{I}}\right), \delta_{0}^{\mathrm{B}, w(\mathfrak{I})}\right\rangle_{\mathrm{B}, \infty}=\int_{0}^{\infty} f_{\mathfrak{I}}(\sqrt{-1} y) d y
$$




\section{T. OCHIAI}

by definition, which makes sense since $f_{\mathfrak{I}}$ is a cuspform. The integration

$$
\int_{0}^{\infty} f_{\mathfrak{I}}(\sqrt{-1} y) d y
$$

is equal to $L\left(f_{\mathfrak{I}}, 1\right) /-2 \pi \sqrt{-1}$ by calculation.

In [Kit94], Kitagawa constructed modules of $\Lambda$-adic modular symbols $\mathcal{B}^{ \pm}$, which have the following properties.

(1) $\mathcal{B}^{ \pm}$is a finitely generated $\mathbb{H}_{\mathcal{F}}^{\text {ord }}$-modules whose generic ranks are one.

(2) For each $\mathfrak{I} \in \mathfrak{X}_{\text {arith }}\left(\mathbb{H}_{\mathcal{F}}^{\text {ord }}\right)_{\geqslant 0}, \mathcal{B}^{ \pm} / \operatorname{Ker}(\mathfrak{I}) \mathcal{B}^{ \pm}$is a lattice of $V_{\mathrm{B}}\left(f_{\mathfrak{I}}\right) \otimes_{\mathbb{Q}_{f_{\mathfrak{I}}}} \widehat{\mathbb{Q}}_{f_{\mathfrak{I}}}$.

Definition 6.6. Let $\mathfrak{I} \in \mathfrak{X}_{\text {arith }}\left(\mathbb{H}_{\mathcal{F}}^{\text {ord }}\right)_{\geqslant 0}$. Then $f_{\mathfrak{I}}$ is a newform in $S_{w(\mathfrak{I})+2}\left(\Gamma_{1}(M)\right)$ for a certain multiple $M$ of $N$. Let $\mathcal{O}_{f_{\mathfrak{I}}}$ be the ring of integers of $\mathbb{Q}_{f_{\mathfrak{I}}}$. Choose an $\mathcal{O}_{f_{\mathfrak{I}}}$-basis $\delta_{\mathfrak{I}}^{\mathrm{B}, \pm}$ of the natural $\mathcal{O}_{f_{\mathfrak{I}}}$-lattice $H_{\mathrm{B}, c}^{1}\left(Y_{1}(M)_{\mathbb{C}}, \operatorname{Sym}^{w(\mathfrak{I})}\left(R^{1} p_{*} \mathcal{O}_{f_{\mathfrak{I}}}\right)\right) \cap V_{\mathrm{B}}\left(f_{\mathfrak{I}}\right)^{ \pm}$of $V_{\mathrm{B}}\left(f_{\mathfrak{I}}\right)^{ \pm}$.

(1) We define a complex period $\Omega_{\infty, \mathfrak{I}}^{ \pm} \in \mathbb{C}$ to be $\Omega_{\infty, \mathfrak{I}}^{ \pm}=\left\langle\operatorname{Per}^{ \pm}\left(\delta_{\mathfrak{I}}^{\mathrm{dR}}\right), \delta_{\mathfrak{I}}^{\mathrm{B}, \pm}\right\rangle_{\mathrm{B}, \infty}$.

(2) Let $\langle\cdot, \cdot\rangle_{\mathrm{B}, p}$ be the extension of $\langle\cdot, \cdot\rangle_{\mathrm{B}}$ as follows:

$$
V_{\mathrm{B}}\left(f_{\mathfrak{I}}\right) \otimes_{\mathbb{Q}_{f}} \overline{\mathbb{Q}}_{p} \times V_{\mathrm{B}}\left(f_{\mathfrak{I}}\right) \otimes_{\mathbb{Q}_{f_{\mathfrak{I}}}} \overline{\mathbb{Q}}_{p} \stackrel{\langle\cdot, \cdot\rangle_{\mathrm{B}, p}}{\longrightarrow} \overline{\mathbb{Q}}_{p} .
$$

We define a $p$-adic period $C_{p, \mathfrak{I}, b}^{ \pm} \in \overline{\mathbb{Q}}_{p}$ as $C_{p, \mathfrak{I}, b}^{ \pm}=\left\langle b_{\mathfrak{I}}^{ \pm}, \delta_{\mathfrak{I}}^{\mathrm{B}, \pm}\right\rangle_{\mathrm{B}, p}$.

Theorem 6.7 [Kit94, Theorem 1.1]. Let us fix an $\mathbb{H}_{\mathcal{F}}^{\text {ord }}$-basis $b$ of $\mathcal{B}^{(-1)^{i-1}}$. Then we have a twovariable $p$-adic $L$-function $L_{p}^{\mathrm{Ki}}(\mathcal{T}) \in \mathbb{H}_{\mathcal{F}}^{\text {n.o }}$ with the following interpolation properties:

$$
\begin{aligned}
& \left(\chi^{j} \eta \circ \mathfrak{I}\right)\left(L_{p, b}^{\mathrm{Ki}}(\mathcal{T})\right) / C_{p, \mathfrak{I}, b}^{(-1)^{i-1}} \\
& \quad=(-1)^{j-1}(j-1) !\left(1-\frac{\left(\omega^{i-j} \eta\right)(p) p^{j-1}}{a_{p}\left(f_{\mathfrak{I}}\right)}\right)\left(\frac{p^{j-1}}{a_{p}\left(f_{\mathfrak{I}}\right)}\right)^{q(i, j, \eta)} G\left(\omega^{j-i} \eta^{-1}\right) \frac{L\left(f_{\mathfrak{I}}, \omega^{i-j} \eta, j\right)}{(2 \pi \sqrt{-1})^{j} \Omega_{\infty, \mathfrak{I}}^{(-1)^{i-1}}},
\end{aligned}
$$

where $q(i, j, \eta)$ is the p-order of the conductor of $\omega^{j-i} \eta^{-1}$ and $G\left(\omega^{j-i} \eta^{-1}\right)$ is the Gauss sum for $\omega^{j-i} \eta^{-1}$.

Remark 6.8.

(1) In general, the complex period is defined to be the determinant of the comparison isomorphism obtained by de Rham's theorem between the Betti realization and the de Rham realization. In our case, the comparison isomorphism $\mathrm{Per}^{ \pm}$for the motive associated to $f_{\mathfrak{I}}$ is an isomorphism between one-dimensional vector spaces. On the side of the de Rham realization, we have the canonical basis obtained by $f_{\mathfrak{I}}$. On the other hand, the Betti realization does not have a canonical basis and our complex period depends on the choice of $\mathcal{O}_{f_{\mathfrak{I}}}$ basis $\delta_{\mathfrak{I}}^{\mathrm{B}, \pm}$. To make the dependence of the complex period $\Omega_{\infty, \mathfrak{I}}^{ \pm}$on the choice of $\delta_{\mathfrak{I}}^{\mathrm{B}, \pm}$ clear, it should have been denoted $\Omega_{\infty, \mathfrak{I}}^{ \pm}\left(\delta_{\mathfrak{I}}^{\mathrm{B}, \pm}\right)$. However, we usually denote it by $\Omega_{\infty, \mathfrak{I}}^{ \pm}$to avoid the complicated notation.

(2) In the interpolation property of $L_{p, b}^{\mathrm{Ki}}(\mathcal{T})$, the $p$-adic error term $C_{p, \Im, b}^{ \pm}$also depends on the choice of $\delta_{\mathfrak{I}}^{\mathrm{B}, \pm}$. When we take another choice of basis ${\delta^{\prime}}_{\mathfrak{I}}^{\mathrm{B}, \pm}$, we have

$$
C_{p, \mathfrak{I}, b}^{ \pm}\left(\delta_{\mathfrak{I}}^{\mathrm{B}, \pm}\right) / C_{p, \mathfrak{I}, b}^{ \pm}\left(\delta_{\mathfrak{I}}^{\prime \mathrm{B}, \pm}\right)=\Omega_{\infty, \mathfrak{I}}^{ \pm}\left(\delta_{\mathfrak{I}}^{\mathrm{B}, \pm}\right) / \Omega_{\infty, \mathfrak{I}}^{ \pm}\left(\delta_{\mathfrak{I}}^{\prime \mathrm{B}, \pm}\right) .
$$

Since the left-hand side and the right-hand side of the equation in Theorem 6.7 behave in the same way when we change $\delta_{\mathfrak{I}}^{\mathrm{B}, \pm}$, the interpolation is well-defined in spite of the ambiguity of the basis $\delta_{\mathfrak{J}}^{\mathrm{B}, \pm}$. 


\section{ON THE TWO-VARIABLE IWASAWA MAIN CONJECTURE}

Proposition 6.9 [Kat04]. Assume Condition (Ir). Let us fix an $\mathbb{H}_{\mathcal{F}}^{\text {ord }}$-basis $b$ of $\mathcal{B}^{(-1)^{i-1}}$ and an $\mathbb{H}_{\mathcal{F}}^{\text {ord }}$-basis $d$ of $\mathcal{D}$. Fix an arithmetic point $\mathfrak{I} \in \mathfrak{X}_{\text {arith }}\left(\mathbb{H}_{\mathcal{F}}^{\text {ord }}\right)$ with $w(\mathfrak{I})=0$. Then we have an Euler system $\left\{\mathcal{Z}_{\mathfrak{I}}(r) \in H^{1}\left(\mathbb{Q}\left(\mu_{r}\right)_{\Sigma} / \mathbb{Q}\left(\mu_{r}\right), \mathcal{T}^{*}(1)_{I}\right)\right\}$ whose first layer $\mathcal{Z}_{\mathfrak{I}}=\mathcal{Z}_{\mathfrak{I}}(1)$ satisfies the following properties.

(1) For each finite order character $\eta$ of $\Gamma$, ( $\left.\exp ^{*} \circ \operatorname{loc}_{/ f}\right)\left(\eta\left(\mathcal{Z}_{\mathfrak{I}}\right)\right)$ is contained in $\mathrm{Fil}^{1} V_{\mathrm{dR}}\left(\bar{f}_{\mathfrak{I}} \otimes\right.$ $\left.\omega^{1-i} \eta^{-1}\right) \subset \mathrm{Fil}^{0} \mathrm{D}_{\mathrm{dR}}\left(V_{f_{\mathfrak{I}}}^{*} \otimes \omega^{1-i} \eta^{-1}\right)$, where $\mathrm{loc}_{/ f}$ is the localization map:

$$
H^{1}\left(\mathbb{Q}_{\Sigma} / \mathbb{Q}, T_{f_{\mathfrak{I}}}^{*}(1) \otimes \omega^{1-i} \eta^{-1}\right) \rightarrow H^{1}\left(\mathbb{Q}_{p}, T_{f_{\mathfrak{I}}}^{*}(1) \otimes \omega^{1-i} \eta^{-1}\right) \rightarrow H_{/ f}^{1}\left(\mathbb{Q}_{p}, T_{f_{\mathfrak{I}}}^{*}(1) \otimes \omega^{1-i} \eta^{-1}\right) .
$$

(2) Further,

$$
\left(\exp ^{*} \circ \operatorname{loc}_{/ f}\right)\left(\eta\left(\mathcal{Z}_{\mathfrak{I}}\right)\right)=\frac{C_{p, \mathfrak{I}, b}^{(-1)^{i-1}}}{C_{p, \mathfrak{I}, d}} \cdot \frac{L_{(p)}\left(f_{\mathfrak{I}}, \omega^{i-1} \eta, 1\right)}{(2 \pi \sqrt{-1})^{j} \Omega_{\infty, \mathfrak{I}}^{(-1)^{i-1}}} \cdot \bar{\delta}_{\mathfrak{I}}^{\mathrm{dR}} .
$$

(We denote by $\mathbb{Q}\left(\mu_{r}\right)_{\Sigma}$ the maximal Galois extension of $\mathbb{Q}\left(\mu_{r}\right)$ unramified outside primes over $\Sigma$.)

Remark 6.10. By taking the projective limit of the elements in Galois cohomology groups obtained via the Chern character from Beilinson-Kato elements in the $K_{2}$-group of $Y_{1}(N p) \otimes \mathbb{Q}\left(\mu_{r p^{s}}\right)$, we have an Euler system $\left\{\mathcal{Z}_{\mathfrak{I}, 0}(r) \in H^{1}\left(\mathbb{Q}\left(\mu_{r}\right)_{\Sigma} / \mathbb{Q}\left(\mu_{r}\right), \mathcal{T}^{*}(1)_{\mathfrak{I}}\right)\right\}$ where $r$ runs square-free natural numbers prime to $p$. The above Euler system $\mathcal{Z}_{\mathfrak{I}}(r)$ is optimally normalized at $\mathfrak{I}$ and is obtained as a summation $\sum_{\xi} c_{\xi} \mathcal{Z}_{\mathfrak{I}, 0}(r)^{\xi}$ multiplied by $C_{p, \mathfrak{I}, b} / C_{p, \mathfrak{I}, d}$, where $\mathcal{Z}_{\mathfrak{I}, 0}(r)^{\xi}$ is the twist of $\mathcal{Z}_{\mathfrak{I}, 0}(r)$ by $\xi \in \mathrm{SL}_{2}(\mathbb{Z})$ and $c_{\xi}$ are rational integers. For such an optimal normalization for a fixed $f_{\mathfrak{I}}$, we refer the reader to $[$ Kat04, $\S 12]$.

We give the following optimization of the two-variable Beilinson-Kato element.

Theorem 6.11. Let us fix an $\mathbb{H}_{\mathcal{F}}^{\text {ord }}$-basis $b$ of $\mathcal{B}^{(-1)^{i}}$ and an $\mathbb{H}_{\mathcal{F}}^{\text {ord }}$-basis $d$ of $\mathcal{D}$. Then we have an Euler system $\left\{\mathcal{Z}^{\mathrm{Ki}}(r) \in H^{1}\left(\mathbb{Q}\left(\mu_{r}\right)_{\Sigma} / \mathbb{Q}\left(\mu_{r}\right), \mathcal{T}^{*}(1)\right)\right\}$ in the sense of Definition 2.2 such that the specialization of the first layer $\mathcal{Z}^{\mathrm{Ki}}=\mathcal{Z}^{\mathrm{Ki}}(1)$ at each arithmetic point $\mathfrak{I} \in \mathfrak{X}_{\text {arith }}\left(\mathbb{H}_{\mathcal{F}}^{\text {ord }}\right)$ with $w(\mathfrak{I})=0$ and at each finite order character $\eta$ of $\Gamma$ satisfies the following properties:

(1) $\left(\exp ^{*} \circ \operatorname{loc}_{/ f}\right)\left((\eta \circ \mathfrak{I})\left(\mathcal{Z}^{\mathrm{Ki}}\right)\right)$ is contained in $\mathrm{Fil}^{1} V_{\mathrm{dR}}\left(\bar{f}_{\mathfrak{I}} \otimes \omega^{1-i} \eta^{-1}\right) \subset \mathrm{Fil}^{0} \mathrm{D}_{\mathrm{dR}}\left(V_{f_{\mathfrak{I}}}^{*} \otimes \omega^{1-i} \eta^{-1}\right)$;

(2) further,

$$
\left(\exp ^{*} \circ \operatorname{loc}_{/ f}\right)\left((\eta \circ \mathfrak{I})\left(\mathcal{Z}^{\mathrm{Ki}}\right)\right)=\frac{C_{p, \mathfrak{I}, b}^{(-1)^{i-1}}}{C_{p, \mathfrak{I}, d}} \cdot \frac{L_{(p)}\left(f_{\mathfrak{I}}, \omega^{i-1} \eta, 1\right)}{(2 \pi \sqrt{-1}) \Omega_{\infty, \mathfrak{I}}^{(-1)^{i-1}}} \cdot \bar{\delta}_{\mathfrak{I}}^{\mathrm{dR}},
$$

where $L_{(p)}\left(f_{\mathfrak{I}}, \omega^{i-1} \eta, s\right)$ is the $\omega^{i-1} \eta$-twist of the Hecke L-function for $f_{\mathfrak{I}}$ whose $p$-factor is removed.

Remark 6.12.

(1) The construction of $\mathcal{Z}^{\mathrm{Ki}}(r)$ will be done by 'gluing' of the elements $\mathcal{Z}_{\mathfrak{I}}(r)$ given in Proposition 6.9 for various $\mathfrak{I} \in \mathfrak{X}_{\text {arith }}\left(\mathbb{H}_{\mathcal{F}}^{\text {ord }}\right)$ with $w(\mathfrak{I})=0$ by using Lemma 6.13 below.

(2) Although the interpolation property is given only for $\mathfrak{I} \in \mathfrak{X}_{\text {arith }}\left(\mathbb{H}_{\mathcal{F}}^{\text {ord }}\right)$ with $w(\mathfrak{I})=0$, $\left(\exp ^{*} \circ\right.$ $\left.\operatorname{loc}_{/ f}\right)((\eta \circ \mathfrak{I})(\mathcal{Z}))$ is related to an optimal $L$-value even when $w(\mathfrak{I})>0$.

Proof of Theorem 6.11. Let $\mathfrak{S}=\left\{I=\operatorname{Ker}(\mathfrak{I}) \mathbb{H}_{\mathcal{F}}^{\text {n.o }} \mid \mathfrak{I} \in \mathfrak{X}_{\text {arith }}\left(\mathbb{H}_{\mathcal{F}}^{\text {ord }}\right), w(\mathfrak{I})=0\right\}$. We denote by $\mathfrak{A}$ a subset of the set of height-one ideals of $\mathbb{H}_{\mathcal{F}}^{\text {n.o }}$ as follows:

$$
\mathfrak{A}=\left\{J=\bigcap_{I \in S} I \mid S \subset \mathfrak{S}, \sharp S<\infty\right\} .
$$

Note that $J \cap J^{\prime} \in \mathfrak{A}$ for any $J, J^{\prime} \in \mathfrak{A}$ and that the intersection $\bigcap J$ for infinitely many $J \in \mathfrak{A}$ is zero. 


\section{T. OCHIAI}

Lemma 6.13. For each natural number $r$ and for each $J, J^{\prime} \in \mathfrak{A}$, we have the exact sequence

$$
0 \longrightarrow H^{1}\left(\mathcal{T}^{*}(1)_{J \cap J^{\prime}}\right) \longrightarrow H^{1}\left(\mathcal{T}^{*}(1)_{J}\right) \oplus H^{1}\left(\mathcal{T}^{*}(1)_{J^{\prime}}\right) \longrightarrow H^{1}\left(\mathcal{T}^{*}(1)_{J+J^{\prime}}\right),
$$

where $H^{1}(M)$ is $H^{1}\left(\mathbb{Q}\left(\mu_{r}\right)_{\Sigma} / \mathbb{Q}\left(\mu_{r}\right), M\right)$ in the above sequence.

In the following, we only construct $\mathcal{Z}^{\mathrm{Ki}}=\mathcal{Z}^{\mathrm{Ki}}(1) \in H^{1}\left(\mathbb{Q}_{\Sigma} / \mathbb{Q}, \mathcal{T}^{*}(1)\right)$ with the two desired properties stated in Theorem 6.11. The construction for general $r$ is done in basically the same way using Lemma 6.13. We need the following claim for the proof.

Claim 6.14. Let $J \in \mathfrak{A}$. Then there exists an element $\mathcal{Z}_{J}$ such that $\left(\exp ^{*} \circ\right.$ loc $\left./ f\right)\left((\eta \circ \mathfrak{I})\left(\mathcal{Z}_{J}\right)\right)$ satisfy the two properties stated in Theorem 6.11 for all arithmetic points $\mathfrak{I} \in \mathfrak{X}_{\text {arith }}\left(\mathbb{H}_{\mathcal{F}}^{\text {ord }}\right)$ with $\operatorname{Ker}(\mathfrak{I}) \mathbb{H}_{\mathcal{F}}^{\text {n.o }} \supset J$ and for all finite order characters $\eta$ of $\Gamma$.

In fact, $\mathcal{Z}$ is obtained as $\lim _{J \in A} \mathcal{Z}_{J} \in H^{1}\left(\mathbb{Q}_{\Sigma} / \mathbb{Q}, \mathcal{T}^{*}(1)\right)$ when $J$ runs a directed subset $A \subset \mathfrak{A}$ such that $\bigcap_{J \in A} J=0$. Hence, we will prove the above claim in the rest of the proof. The proof proceeds by induction with respect to the numbers of arithmetic points $\mathfrak{I} \in \mathfrak{X}_{\text {arith }}\left(\mathbb{H}_{\mathcal{F}}^{\text {ord }}\right)$ with $\operatorname{Ker}(\mathfrak{I}) \mathbb{H}_{\mathcal{F}}^{\text {n.o }} \supset J$. By Proposition 6.9 the claim holds when $J=\operatorname{Ker}(\mathfrak{I}) \mathbb{H}_{\mathcal{F}}^{\text {n.o }}$ for an arithmetic point $\mathfrak{I} \in \mathfrak{X}_{\text {arith }}\left(\mathbb{H}_{\mathcal{F}}^{\text {ord }}\right)$. Now we take arbitrary ideal $J \in \mathfrak{A}$ at which Claim 6.14 is true. We will prove Claim 6.14 for $J \cap I$ where $I=\operatorname{Ker}(\mathfrak{I}) \mathbb{H}_{\mathcal{F}}^{\text {n.o }}$ for an arithmetic point $\mathfrak{I} \in \mathfrak{X}_{\text {arith }}\left(\mathbb{H}_{\mathcal{F}}^{\text {ord }}\right)$ such that $w(\mathfrak{I})=0$ and $J \not \subset I$. Let us denote $\mathcal{T}^{*} /(J, \operatorname{Ker}(\eta)) \mathcal{T}^{*}$ by $T_{J, \eta}^{*}$ and let us denote the continuous Galois cohomology $H^{1}\left(\mathbb{Q}_{\Sigma} / \mathbb{Q}, M\right)$ by $H^{1}(M)$ for short. Then, we have the following diagram for each finite order character $\eta$ of $\Gamma$ :

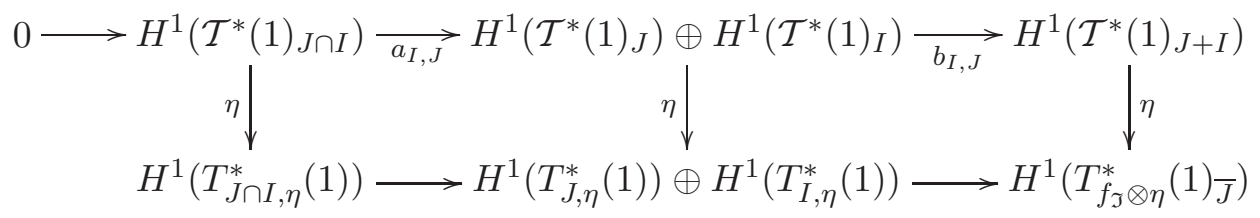

where $a_{I, J}$ sends $x \in H^{1}\left(\mathcal{T}^{*}(1)_{J \cap I}\right)$ to $x_{J} \oplus x_{I} \in H^{1}\left(\mathcal{T}^{*}(1)_{J}\right) \oplus H^{1}\left(\mathcal{T}^{*}(1)_{I}\right)$ and $b_{I, J}$ sends $x \oplus y \in$ $H^{1}\left(\mathcal{T}^{*}(1)_{J}\right) \oplus H^{1}\left(\mathcal{T}^{*}(1)_{I}\right)$ to $x_{J+I}-y_{J+I} \in H^{1}\left(\mathcal{T}^{*}(1)_{J+I}\right)$. Let us consider the following morphism:

$$
H^{1}\left(\mathbb{Q} \Sigma / \mathbb{Q}, T_{f_{\mathfrak{I}} \otimes \eta}^{*}(1)\right) \stackrel{\mathrm{loc} / f}{\longrightarrow} \frac{H^{1}\left(\mathbb{Q}_{p}, T_{f_{\mathfrak{I}} \otimes \eta}^{*}(1)\right)}{H_{f}^{1}\left(\mathbb{Q}_{p}, T_{f_{\mathfrak{I}} \otimes \eta}^{*}(1)\right)} \stackrel{\langle d,\rangle_{\mathrm{dR}} \circ \exp ^{*}}{\longrightarrow}(\eta \circ \mathfrak{I})\left(\mathbb{H}_{\mathcal{F}}^{\text {n.o }}\right) .
$$

The element $\left(\eta \circ \mathfrak{I}^{\prime}\right)\left(\mathcal{Z}_{J}\right) \in H^{1}\left(\mathbb{Q}_{\Sigma} / \mathbb{Q}, T_{f_{\mathfrak{I}^{\prime}} \otimes \eta}^{*}(1)\right)$ (respectively $\eta\left(\mathcal{Z}_{I}\right) \in H^{1}\left(\mathbb{Q}_{\Sigma} / \mathbb{Q}, T_{f_{\mathfrak{I}} \otimes \eta}^{*}(1)\right)$ ) is mapped to

$$
v_{\mathfrak{I}^{\prime}, \eta}:=\frac{C_{p, \mathfrak{I}^{\prime}, b}^{(-1)^{i-1}}}{C_{p, \mathfrak{I}^{\prime}, d}} \frac{L_{(p)}\left(f_{\mathfrak{I}^{\prime}}, \eta, 1\right)}{(2 \pi \sqrt{-1}) \Omega_{\infty, \mathfrak{I}^{\prime}}^{(-1)^{i-1}}}
$$

when $J \subset \operatorname{Ker}\left(\mathfrak{I}^{\prime}\right) \mathbb{H}_{\mathcal{F}}^{\text {n.o }}$ (respectively $\mathfrak{I}=\mathfrak{I}^{\prime}$ ). The following lemma is obtained by the Euler system argument using the Beilinson-Kato element and by a result of Rohrlich (cf. [Kat04]).

Lemma 6.15. Under Condition (Ir), the map in (11) is injective when the conductor of $\eta$ is sufficiently large.

In fact, since $H^{1}\left(\mathbb{Q}_{\Sigma} / \mathbb{Q}, T_{f_{\mathfrak{I}} \otimes \eta}^{*}(1)\right)$ has no non-zero torsion by Condition (Ir), the kernel of (11) is non-zero if and only if $\mathrm{Sel}_{T_{f_{\mathfrak{J}} \otimes \eta}^{*}}(1)$ is an infinite abelian group. This happens only for finitely many $\eta$ by Kato-Rubin and Rohrlich.

Since values $v_{\mathfrak{I}^{\prime}, \eta}$ and $v_{\mathfrak{I}^{\prime \prime}, \eta}$ are congruent to each other modulo $\left(\operatorname{Ker}\left(\mathfrak{I}^{\prime}\right)+\operatorname{Ker}\left(\mathfrak{I}^{\prime \prime}\right), \operatorname{Ker}(\eta)\right)$, $\eta \circ b_{I, J}\left(\mathcal{Z}_{J} \oplus \mathcal{Z}_{I}\right)$ is zero for each finite order character $\eta$ of $\Gamma$ with sufficiently large conductor. 


\section{ON THE TWO-VARIABLE IWASAWA MAIN CONJECTURE}

Lemma 6.16. Let $I$ be a height-one ideal of $\mathbb{H}_{\mathcal{F}}^{\text {n.o }}$ generated by a height-one ideal of $\mathbb{H}_{\mathcal{F}}^{\text {ord }}$. Then the intersection $\bigcap_{\eta} \operatorname{Ker}(\eta) \subset H^{1}\left(\mathbb{Q}_{\Sigma} / \mathbb{Q}, \mathcal{T}^{*}(1)_{I}\right)$ is trivial when $\eta$ runs infinitely many finite order characters of $\Gamma$.

Since $\mathcal{Z}_{J} \oplus \mathcal{Z}_{I}$ is mapped to zero via $b_{I, J}$ by Lemmas 6.15 and 6.16 , we have an element $\mathcal{Z}_{J \cap I} \in$ $H^{1}\left(\mathbb{Q}_{\Sigma} / \mathbb{Q}, \mathcal{T}^{*}(1)_{J \cap I}\right)$ such that $a_{I, J}\left(\mathcal{Z}_{J \cap I}\right)=\mathcal{Z}_{J} \oplus \mathcal{Z}_{I}$. By construction, $\mathcal{Z}_{J \cap I}$ satisfies the desired properties for Claim 6.14. This completes the proof.

COROLlary 6.17. Let us fix an $\mathbb{H}_{\mathcal{F}}^{\text {ord }}$-basis $b$ of $\mathcal{B}^{(-1)^{i}}$ and an $\mathbb{H}_{\mathcal{F}}^{\text {ord }}$-basis $d$ of $\mathcal{D}$. Then we have an Euler system $\left\{\mathcal{Z}_{b, d}^{\mathrm{Ki}}(r) \in H^{1}\left(\mathbb{Q}\left(\mu_{r}\right)_{\Sigma} / \mathbb{Q}\left(\mu_{r}\right), \mathcal{T}^{*}(1)\right)\right\}$ such that $\Xi_{d}\left(\mathcal{Z}_{b, d}^{\mathrm{Ki}}(1)\right) \in \mathbb{H}_{\mathcal{F}}^{\text {n.o }}$ is equal to the two-variable $p$-adic $L$-function $L_{p, b}^{\mathrm{Ki}}(\mathcal{T})$ of Kitagawa.

\section{IMCs for various specializations of $\mathcal{T}$}

In this section, we formulate and discuss the IMC for various one-variable specializations $\mathcal{T}_{J}$ of $\mathcal{T}$. In particular, we will discuss how to obtain a result on the one-variable Iwasawa theory on $\mathcal{T}_{J}$ from the two-variable Iwasawa theory on $\mathcal{T}$ and vice versa. Recall the following definition.

Definition 7.1. Let $R$ be a Noetherian local domain such that $R$ is integrally closed in the fraction field $\operatorname{Frac}(R)$ of $R$. A finitely generated torsion $R$-module $M$ is called pseudo-null if length $R_{\mathfrak{r}}\left(M_{\mathfrak{l}}\right)=0$ for every height-one prime $\mathfrak{l}$ in $R$ or, equivalently, $\operatorname{Supp}_{R}(M)$ has codimension greater than one in $\operatorname{Spec}(R)$. For a finitely generated $R$-module $M$, we denote by $M_{\text {null }}$ the largest pseudo-null $R$ submodule of $M$.

The difference between $\operatorname{Sel}_{\mathcal{T}}[J]$ and $\operatorname{Sel}_{\mathcal{T}_{J}}$ is an obstruction to the study of the relation between the two-variable IMC for $\mathcal{T}$ and the one-variable IMC for each $\mathcal{T}_{J}$. Thus, we prepare the following lemma which ensures that the term $\left(\operatorname{Sel}_{\mathcal{T}}\right)_{\text {null }}^{\vee} / J\left(\operatorname{Sel}_{\mathcal{T}}\right)_{\text {null }}^{\vee}$ which causes the above obstruction is trivial in our case.

Lemma 7.2. Assume Conditions (Ir) and (Nor) for $\mathcal{T}=\mathcal{T}_{\mathcal{F}}^{(i)}$. Let us consider height-one primes $J \subset \mathbb{H}_{\mathcal{F}}^{\text {n.o }}$ in one of the following three cases:

(a) $J$ is equal to $I=\operatorname{Ker}(\mathfrak{I}) \mathbb{H}_{\mathcal{F}}^{\text {n.o }}$ for certain $\mathfrak{I} \in \mathfrak{X}_{\text {arith }}\left(\mathbb{H}_{\mathcal{F}}^{\text {ord }}\right)_{\geqslant 0}$;

(b) $J$ is equal to $(\gamma-\chi(\gamma)) \subset \mathbb{H}_{\mathcal{F}}^{\text {n.o }}$;

(c) $J$ is equal to $\left(\gamma-\kappa\left(\gamma^{\prime}\right) \gamma^{\prime}\right) \subset \mathbb{H}_{\mathcal{F}}^{\text {n.o }}$.

In case (a), we assume that the module $U_{\mathfrak{I}}$ in Proposition 5.2 is trivial (we do not need an assumption in the other cases).

Then, $\left(\operatorname{Sel}_{\mathcal{T}}\right)_{\text {null }}^{\vee} / J\left(\operatorname{Sel}_{\mathcal{T}}\right)_{\text {null }}^{\vee}$ is a pseudo-null $\mathbb{H}_{\mathcal{F}}^{\text {n.o }} / J$-module.

Proof. Let us consider the following diagram:

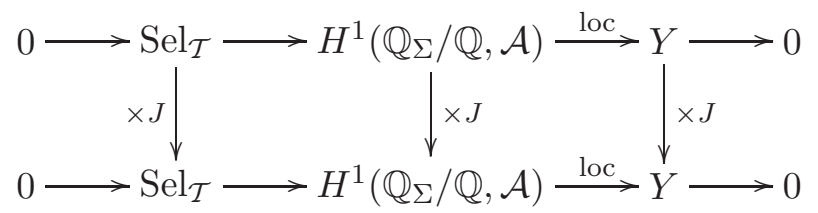

where

$$
Y=\frac{H^{1}\left(\mathbb{Q}_{p}, \mathcal{A}\right)}{H_{\mathrm{Gr}}^{1}\left(\mathbb{Q}_{p}, \mathcal{A}\right)} \oplus \bigoplus_{v \in \Sigma \backslash\{p, \infty\}} \frac{H^{1}\left(\mathbb{Q}_{v}, \mathcal{A}\right)}{H_{\mathrm{ur}}^{1}\left(\mathbb{Q}_{v}, \mathcal{A}\right)}
$$




\section{T. OCHIAI}

The cokernel of the middle vertical map is a subgroup of $H^{2}\left(\mathbb{Q}_{\Sigma} / \mathbb{Q}, \mathcal{A}[J]\right)$, which is zero since $\mathrm{Sel}_{\mathcal{T} / J \mathcal{T}}$ is a cotorsion $\mathcal{O}_{\mathfrak{I}}[[\Gamma]]$-module. By the snake lemma, we have

$$
\left(\operatorname{Sel}_{\mathcal{T}}\right) / J\left(\operatorname{Sel}_{\mathcal{T}}\right) \cong \operatorname{Coker}\left[H^{1}\left(\mathbb{Q}_{\Sigma} / \mathbb{Q}, \mathcal{A}\right)[J] \stackrel{\operatorname{loc}[J]}{\longrightarrow} Y[J]\right] .
$$

We compare Coker(loc $[J])$ with the cokernel of

$$
H^{1}(\mathbb{Q} \Sigma / \mathbb{Q}, \mathcal{A}[J]) \stackrel{\operatorname{loc}_{J}}{\longrightarrow} Y_{J}
$$

where

$$
Y_{J}=\frac{H^{1}\left(\mathbb{Q}_{p}, \mathcal{A}[J]\right)}{H_{\mathrm{Gr}}^{1}\left(\mathbb{Q}_{p}, \mathcal{A}[J]\right)} \oplus \bigoplus_{v \in \Sigma \backslash\{p, \infty\}} \frac{H^{1}\left(\mathbb{Q}_{v}, \mathcal{A}[J]\right)}{H_{\mathrm{ur}}^{1}\left(\mathbb{Q}_{v}, \mathcal{A}[J]\right)} .
$$

By Corollary 4.12, Coker $\left(\operatorname{loc}_{J}\right)$ is zero in cases (a), (b) and (c). Let us admit the following claim for a while.

Claim 7.3. The natural map $s_{J}: Y_{J} \longrightarrow Y[J]$ is surjective.

Let us consider the following diagram.

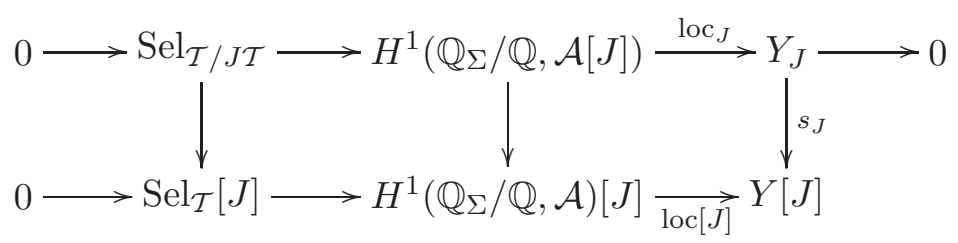

Since $s_{J} \circ \operatorname{loc}_{J}$ is surjective by Claim 7.3, $\operatorname{loc}[J]$ has to be surjective. We have shown that $\left(\operatorname{Sel}_{\mathcal{T}}\right) / J\left(\operatorname{Sel}_{\mathcal{T}}\right)$ is zero or, equivalently, we have shown that $\left(\operatorname{Sel}_{\mathcal{T}}\right)^{\vee}[J]$ is zero by taking the Pontryagin dual. This implies $\left(\mathrm{Sel}_{\mathcal{T}}\right)_{\text {null }}^{\vee}[J]=0$. By [Och05, Lemma 3.1], $M / J M$ is a pseudo-null $\mathbb{H}_{\mathcal{F}}^{\text {n.o }} / J$ module if and only if $M[J]$ is a pseudo-null $\mathbb{H}_{\mathcal{F}}^{\text {n.o }} / J$-module for every pseudo-null $\mathbb{H}_{\mathcal{F}}^{\text {n.o }}$-module $M$. Hence, $\left(\operatorname{Sel}_{\mathcal{T}}\right)_{\text {null }}^{\vee} / J\left(\operatorname{Sel}_{\mathcal{T}}\right)_{\text {null }}^{\vee}$ must be a pseudo-null $\mathbb{H}_{\mathcal{F}}^{\text {n.o }} / J$-module.

We will show Claim 7.3 in the rest of the proof. We have the following exact sequence by using the snake lemma:

$$
0 \longrightarrow H_{\mathrm{Gr}}^{1}\left(\mathbb{Q}_{p}, \mathcal{A}\right)[J] \longrightarrow H^{1}\left(\mathbb{Q}_{p}, \mathcal{A}\right)[J] \stackrel{t_{p}}{\longrightarrow} \frac{H^{1}\left(\mathbb{Q}_{p}, \mathcal{A}\right)}{H_{\mathrm{Gr}}^{1}\left(\mathbb{Q}_{p}, \mathcal{A}\right)}[J] \longrightarrow H_{\mathrm{Gr}}^{1}\left(\mathbb{Q}_{p}, \mathcal{A}\right) / J H_{\mathrm{Gr}}^{1}\left(\mathbb{Q}_{p}, \mathcal{A}\right)
$$

Since $H_{\mathrm{Gr}}^{1}\left(\mathbb{Q}_{p}, \mathcal{A}\right)^{\vee}$ has no torsion $\mathbb{H}_{\mathcal{F}}^{\text {n.o }}$-submodule, the map $t_{p}$ must be surjective. Similarly, we have the following exact sequence for every $v \in \Sigma \backslash\{p, \infty\}$ :

$$
0 \longrightarrow H_{\mathrm{ur}}^{1}\left(\mathbb{Q}_{v}, \mathcal{A}\right)[J] \longrightarrow H^{1}\left(\mathbb{Q}_{v}, \mathcal{A}\right)[J] \stackrel{t_{v}}{\longrightarrow} \frac{H^{1}\left(\mathbb{Q}_{v}, \mathcal{A}\right)}{H_{\mathrm{ur}}^{1}\left(\mathbb{Q}_{v}, \mathcal{A}\right)}[J] \longrightarrow H_{\mathrm{ur}}^{1}\left(\mathbb{Q}_{v}, \mathcal{A}\right) / J H_{\mathrm{ur}}^{1}\left(\mathbb{Q}_{v}, \mathcal{A}\right) .
$$

The Pontryagin dual of the last term is $U_{\mathfrak{I}}$ in case (a). Hence, $t_{v}$ is surjective for every $v \in \Sigma \backslash\{p, \infty\}$ by the assumption of Lemma 7.2. In cases (b) and (c), $t_{v}$ is surjective for every $v \in \Sigma \backslash\{p, \infty\}$ without any assumption. Finally, we have the following commutative diagram.

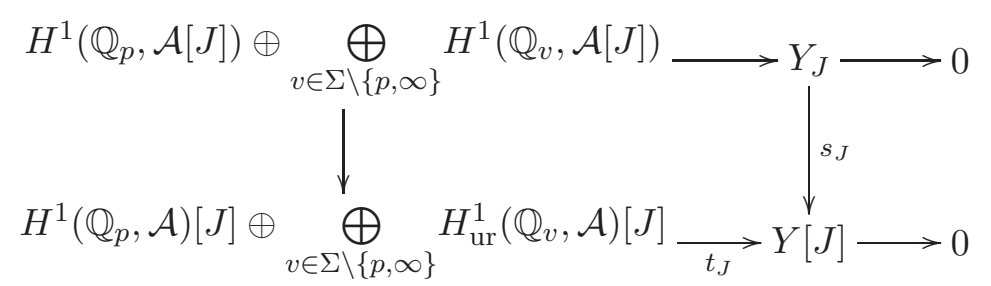

The bottom horizontal map $t_{J}$ is equal to $t_{p} \oplus \bigoplus_{v \in \Sigma \backslash\{p, \infty\}} t_{v}$, which is surjective as shown above. The left vertical map is surjective by definition. Since the square in the diagram is commutative, $s_{J}$ must be surjective. This completes the proof of Claim 7.3. 


\section{ON THE TWO-VARIABLE IWASAWA MAIN CONJECTURE}

\section{(a) IMC for $\mathcal{T}_{I}$}

Let $I=\operatorname{Ker}(\mathfrak{I}) \mathbb{H}_{\mathcal{F}}^{\text {n.o }}$ with $\mathfrak{I} \in \mathfrak{X}_{\text {arith }}\left(\mathbb{H}_{\mathcal{F}}^{\text {ord }}\right)_{\geqslant 0}$. The specialization of $\mathcal{T}$ at $I$ is the cyclotomic deformation of $f_{\mathfrak{I}}$ as we saw in $\S 1$. By Mazur-Tate-Teitelbaum, we have $L_{p}^{\mathrm{MTT}}\left(\mathcal{T}_{I}\right) \in \mathbb{H}_{\mathcal{F}}^{\text {n.o }} / I$ which has the following interpolation property for each finite order character $\eta$ of $\Gamma$ and for each integer $1 \leqslant j \leqslant w(\mathfrak{I})+1:$

$$
\chi^{j} \eta\left(L_{p}^{\mathrm{MTT}}\left(\mathcal{T}_{I}\right)\right)=\left(1-\frac{\left(\omega^{i-j} \eta\right)(p) p^{j-1}}{a_{p}\left(f_{\mathfrak{I}}\right)}\right)\left(\frac{p^{j-1}}{a_{p}\left(f_{\mathfrak{I}}\right)}\right)^{q(i, j, \eta)} G\left(\omega^{j-i} \eta\right) \frac{L\left(f_{\mathfrak{I}}, \omega^{i-j}, j\right)}{(2 \pi \sqrt{-1})^{j-1} \Omega_{\infty, \mathfrak{I}}^{ \pm}},
$$

where $C_{\infty, \mathfrak{I}}^{ \pm}$is a complex period given by Definition 6.6. Note that the ideal $\left(L_{p}^{\mathrm{MTT}}\left(\mathcal{T}_{I}\right)\right)$ is welldefined since $C_{\infty, \mathfrak{I}}^{ \pm}$is unique up to multiplication by a unit in $\mathcal{O}_{f_{\mathfrak{I}}}$. Recall that $\operatorname{Sel}_{I}^{\vee}$ is a cotorsion $\mathbb{H}_{\mathcal{F}}^{\text {n.o }} / I$-module (cf. $\left.\S 4.2\right)$.

ConjeCture 7.4. Let $I=\operatorname{Ker}(\mathfrak{I}) \mathbb{H}_{\mathcal{F}}^{\text {n.o }}$ with $\mathfrak{I} \in \mathfrak{X}_{\text {arith }}\left(\mathbb{H}_{\mathcal{F}}^{\text {ord }}\right)_{\geqslant 0}$. We have the following equality:

$$
\operatorname{length}_{\left(\mathbb{H}_{\mathcal{F}}^{\text {n.o }} / I\right)_{\mathfrak{l}}}\left(\operatorname{Sel}_{I}^{\vee}\right)_{\mathfrak{l}}=\operatorname{ord}_{\mathfrak{l}}\left(L_{p}^{\mathrm{MTT}}\left(\mathcal{T}_{I}\right)\right),
$$

for each height-one prime $\mathfrak{l}$ of $\mathbb{H}_{\mathcal{F}}^{\text {n.o }} / I$.

As a corollary of Theorem 3 in $\S 2$, we have the following result.

Corollary 7.5. Let $I=\operatorname{Ker}(\mathfrak{I}) \mathbb{H}_{\mathcal{F}}^{\text {n.o }}$ with $\mathfrak{I} \in \mathfrak{X}_{\text {arith }}\left(\mathbb{H}_{\mathcal{F}}^{\text {ord }}\right)_{\geqslant 0}$. Assume the same conditions as those in Theorem 2. Suppose further that $P_{\tau}$ which appeared in condition (i) of Theorem 2 is a unit in $\mathbb{H}_{\mathcal{F}}^{\text {n.o }}$ and that $\left(\left(\mathbb{T}_{\mathcal{F}}^{\text {ord }}\right)^{*}\right)_{I_{v}}[\operatorname{Ker}(\mathfrak{I})]$ is trivial for every $\mathfrak{I} \in \mathfrak{X}_{\text {arith }}\left(\mathbb{H}_{\mathcal{F}}^{\text {ord }}\right)_{\geqslant 0}$ and for every $v \in \Sigma \backslash\{p, \infty\}$. Then, the following statements are equivalent.

(1) The two-variable IMC holds for $\mathcal{T}$.

(2) The cyclotomic IMC (cf. Conjecture 7.4) formulated by Mazur-Tate-Teitelbaum holds for every specialization $f_{\mathfrak{I}}$ of $\mathcal{F}$ with $\mathfrak{I} \in \mathfrak{X}_{\text {arith }}\left(\mathbb{H}_{\mathcal{F}}^{\text {ord }}\right)_{\geqslant 0}$.

(3) There exists an $\mathfrak{I}_{0} \in \mathfrak{X}_{\text {arith }}\left(\mathbb{H}_{\mathcal{F}}^{\text {ord }}\right)_{\geqslant 0}$ where the cyclotomic IMC holds for $f_{\mathfrak{I}_{0}}$.

Remark 7.6. Concerning the condition that $\left(\left(\mathbb{T}_{\mathcal{F}}^{\text {ord }}\right)^{*}\right)_{I_{v}}[\operatorname{Ker}(\mathfrak{I})]$ is trivial for $v \in \Sigma \backslash\{p, \infty\}$, we refer to Theorem 3.3 for detailed information on when it is trivial (cf. Remark 4.5). For example, it is not difficult to see that $\left(\left(\mathbb{T}_{\mathcal{F}}^{\text {ord }}\right)^{*}\right)_{I_{v}}[\operatorname{Ker}(\mathfrak{I})]$ is trivial when $\operatorname{ord}_{v}(N) \leqslant 1$ of the image of the local monodromy at $v$ is finite, where $N$ is the tame conductor of $\mathcal{F}$. The condition on $P_{\tau}$ also holds in fairly general situations thanks to the study of the local Galois image by Serre and others (see also the discussion for a concrete example given in $\S 9$ ). We expect that the conclusion of Corollary 7.5 holds without any assumption. However, we cannot prove it at the moment.

Proof of Corollary 7.5. The restriction map $\operatorname{Sel}_{I} \longrightarrow \mathrm{Sel}_{\mathcal{T}}[I]$ is an isomorphism by Proposition 5.2 and by the assumption of Corollary 7.5. Hence, we have

$$
\operatorname{length}_{\left(\mathbb{H}_{\mathcal{F}}^{\text {n.o }} / I\right)_{\mathfrak{r}}}\left(\left(\operatorname{Sel}_{I}\right)^{\vee}\right)_{\mathfrak{r}}=\operatorname{length}_{\left(\mathbb{H}_{\mathcal{F}}^{\text {n.o }} / I\right)_{\mathfrak{r}}}\left(\left(\operatorname{Sel}_{\mathcal{T}}\right)^{\vee} / I\left(\operatorname{Sel}_{\mathcal{T}}\right)^{\vee}\right)_{\mathfrak{r}}
$$

for every height-one prime $\mathfrak{l}$ of $\mathbb{H}_{\mathcal{F}}^{\text {n.o }} / I$. Let $\mathfrak{l}$ be a height-one prime of $\mathbb{H}_{\mathcal{F}}^{\text {n.o }} / I$ and $\widetilde{\mathfrak{l}}$ be a height-one prime of $\mathbb{H}_{\mathcal{F}}^{\text {n.o }}$ which is the pre-image of $\mathfrak{l}$ via $\mathbb{H}_{\mathcal{F}}^{\text {n.o }} \rightarrow \mathbb{H}_{\mathcal{F}}^{\text {n.o }} / I$. Then, we have

$$
\operatorname{length}_{\left(\mathbb{H}_{\mathcal{F}}^{\text {n.o }} / I\right)_{\mathfrak{r}}}\left(\left(\operatorname{Sel}_{\mathcal{T}}\right)^{\vee} / I\left(\operatorname{Sel}_{\mathcal{T}}\right)^{\vee}\right)_{\mathfrak{r}}=\operatorname{length}_{\left(\mathbb{H}_{\mathcal{F}}^{\text {n.o }}\right)_{\mathfrak{r}}}\left(\left(\operatorname{Sel}_{\mathcal{T}}\right)^{\vee}\right)_{\mathfrak{l}}
$$

by Lemma 7.2. On the other hand, since the $p$-adic period $C_{p, \mathfrak{I}, b}$ is known to be a $p$-adic unit by Kitagawa's construction, we have

$$
\operatorname{ord}_{\left(\mathbb{H}_{\mathcal{F}}^{\text {n.o }} / I\right)_{\mathfrak{r}}}\left(L_{p}^{\mathrm{MTT}}\left(\mathcal{T}_{I}\right)\right)=\operatorname{ord}_{\left(\mathbb{H}_{\mathcal{F}}^{\text {n.o }} / I\right)_{\mathfrak{r}}}\left(L_{p}^{\mathrm{Ki}}(\mathcal{T}) \bmod I\right)
$$

for every height-one prime $\mathfrak{l}$ of $\mathbb{H}_{\mathcal{F}}^{\text {n.o }} / I$. Thus, we have proved the implication $1 \Longrightarrow 2$. The implication $2 \Longrightarrow 3$ is trivial. Finally, we prove the implication $3 \Longrightarrow 1$. We have the inequality

$$
\operatorname{length}_{\left(\mathbb{H}_{\mathcal{F}}^{\text {n.o }}\right)_{\mathfrak{l}}}\left(\operatorname{Sel}_{\mathcal{T}}\right)_{\mathfrak{l}}^{\vee} \leqslant \operatorname{ord}_{\left(\mathbb{H}_{\mathcal{F}}^{\text {noo }}\right)_{\mathfrak{l}}}\left(L_{p}^{\mathrm{Ki}}(\mathcal{T})\right)
$$




\section{T. OCHIAI}

for every height-one prime $\widetilde{\mathfrak{l}}$ of $\mathbb{H}_{\mathcal{F}}^{\text {n.o }}$ by Theorem 3 in $\S 2$. By the assumption of the third assertion, we have the following equality:

$$
\operatorname{length}_{\left(\mathbb{H}_{\mathcal{F}}^{\text {n.o }} / I_{0}\right)_{\mathfrak{r}}}\left(\left(\operatorname{Sel}_{\mathcal{T}}\right)^{\vee} / I_{0}\left(\operatorname{Sel}_{\mathcal{T}}\right)^{\vee}\right)_{\mathfrak{l}}=\operatorname{ord}_{\left(\mathbb{H}_{\mathcal{F}}^{\text {n.o }} / I_{0}\right)_{\mathfrak{r}}}\left(L_{p}^{\mathrm{Ki}}(\mathcal{T})\right)
$$

for every height-one prime $\mathfrak{l}$ of $\mathbb{H}_{\mathcal{F}}^{\text {n.o }} / I_{0}$, where $I_{0}=\operatorname{Ker}\left(\mathfrak{I}_{0}\right) \mathbb{H}_{\mathcal{F}}^{\text {n.o }}$. For each height-one prime $\widetilde{\mathfrak{l}}$ of $\mathbb{H}_{\mathcal{F}}^{\text {n.o }}$, it is not difficult to see that we have the equality

$$
\operatorname{length}_{\left(\mathbb{H}_{\mathcal{F}}^{\text {n.o }}\right)_{\mathfrak{l}}}\left(\operatorname{Sel}_{\mathcal{T}}\right)_{\mathfrak{l}}^{\vee}=\operatorname{ord}_{\left(\mathbb{H}_{\mathcal{F}}^{\text {n.o }}\right)_{\mathfrak{l}}}\left(L_{p}^{\mathrm{Ki}}(\mathcal{T})\right)
$$

by combining the inequality $(15)$ and the equality (16) for a height-one prime $\mathfrak{l}$ of $\mathbb{H}_{\mathcal{F}}^{\text {n.o }} / I_{0}$ so that $\widetilde{\mathfrak{l}}$ is the pre-image of $\mathfrak{l}$ via $\mathbb{H}_{\mathcal{F}}^{\text {n.o }} \rightarrow \mathbb{H}_{\mathcal{F}}^{\text {n.o }} / I$. Thus, we complete the proof.

(b) IMC for $\mathcal{T}_{(\gamma-\chi(\gamma))}$

Following $\S 4.2, \operatorname{Sel}_{(\gamma-\chi(\gamma))}$ is a cotorsion $\mathbb{H}_{\mathcal{F}}^{\text {ord }}$-module. On the other hand, we define $L_{p}\left(\mathcal{T}_{(\gamma-\chi(\gamma))}\right)$ to be the image of $L_{p}^{\mathrm{Ki}}(\mathcal{T})$ in $\mathbb{H}_{\mathcal{F}}^{\text {n.o }} /(\gamma-\chi(\gamma))=\mathbb{H}_{\mathcal{F}}^{\text {ord }}$. The one-variable IMC is formulated as follows.

ConjeCture 7.7. We have the following equality:

$$
\operatorname{length}_{\left(\mathbb{H}_{\mathcal{F}}\right)_{\mathfrak{l}}}\left(\operatorname{Sel}_{(\gamma-\chi(\gamma))}^{\vee}\right)_{\mathfrak{l}}=\operatorname{ord}_{\mathfrak{l}}\left(L_{p}\left(\mathcal{T}_{(\gamma-\chi(\gamma))}\right)\right) \text {, }
$$

for each height-one prime $\mathfrak{l}$ of $\mathbb{H}_{\mathcal{F}}^{\text {ord }}$.

We have the following corollary of Theorem 3 in $\S 2$.

Corollary 7.8. We have the following.

(1) The two-variable main conjecture (Conjecture 2.4) implies Conjecture 7.7.

(2) Assume further the conditions listed in Theorem 2 in $\S 2$. with $P_{\tau}$ a unit in $\mathbb{H}_{\mathcal{F}}^{\text {n.o }}$. Then Conjecture 7.7 implies the two-variable IMC (Conjecture 2.4).

This is proved in the same manner as case (a) above by using Lemma 7.2.

(c) IMC for $\mathcal{T}_{\left(\gamma-\kappa\left(\gamma^{\prime}\right) \gamma^{\prime}\right)}$

Following $\S 4.2, \operatorname{Sel}_{\left(\gamma-\kappa\left(\gamma^{\prime}\right) \gamma^{\prime}\right)}$ is a cotorsion $\mathbb{H}_{\mathcal{F}}^{\text {ord }}$-module. On the other hand, we define $L_{p}\left(\mathcal{T}_{\left(\gamma-\kappa\left(\gamma^{\prime}\right) \gamma^{\prime}\right)}\right)$ to be the image of $L_{p}^{\mathrm{Ki}}(\mathcal{T})$ in $\mathbb{H}_{\mathcal{F}}^{\text {n.o }} /\left(\left(\gamma-\kappa\left(\gamma^{\prime}\right) \gamma^{\prime}\right)\right)=\mathbb{H}_{\mathcal{F}}^{\text {ord }}$.

Conjecture 7.9. Let $\mathcal{T}=\mathcal{T}_{\mathcal{F}}^{(i)}$. We have the following equality:

$$
\operatorname{length}_{\left(\mathbb{H}_{\mathcal{F}}^{\text {ord }}\right)_{\mathfrak{l}}}\left(\operatorname{Sel}_{\left(\gamma-\kappa\left(\gamma^{\prime}\right) \gamma^{\prime}\right)}^{\vee}\right)_{\mathfrak{l}}+e_{\mathfrak{l}}=\operatorname{ord}_{\mathfrak{l}}\left(L_{p}\left(\mathcal{T}_{\left(\gamma-\kappa\left(\gamma^{\prime}\right) \gamma^{\prime}\right)}\right)\right) \text {, }
$$

for each height-one prime $\mathfrak{l}$ of $\mathbb{H}_{\mathcal{F}}^{\text {ord }}$, where

$$
e_{\mathfrak{l}}= \begin{cases}\operatorname{ord}_{\mathfrak{l}}\left(1-A_{p}(\mathcal{F})\right) & \text { if } \mathrm{F}^{-} \mathcal{A}[\mathfrak{M}]^{I_{p}} \neq 0, \\ 0 & \text { if } \mathrm{F}^{-} \mathcal{A}[\mathfrak{M}]^{I_{p}}=0 .\end{cases}
$$

A corollary of Theorem 3 in $\S 2$ is given as follows.

Corollary 7.10. We have the following.

(i) The two-variable main conjecture (Conjecture 2.4) implies Conjecture 7.9.

(ii) Assume further the conditions listed in Theorem 2 of $\S 2$ with $P_{\tau}$ a unit in $\mathbb{H}_{\mathcal{F}}^{\text {n.o }}$. Then Conjecture 7.9 implies the two-variable IMC (Conjecture 2.4).

This is proved in the same manner as cases (a) and (b) by using Lemma 7.2. 


\section{ON THE TWO-VARIABLE IWASAWA MAIN CONJECTURE}

\section{(d) IMC conjecture for $\mathcal{T}_{\left(\gamma^{2}-\kappa^{2}\left(\gamma^{\prime}\right) \gamma^{\prime}\right)}$}

The Selmer group for the diagonal specialization $\mathcal{T}_{\left(\gamma^{2}-\kappa^{2}\left(\gamma^{\prime}\right) \gamma^{\prime}\right)}$ is not a cotorsion $\mathbb{H}_{\mathcal{F}}^{\text {ord }}$-module in general.

ConjeCture 7.11. We have the following.

(1) Let $\mathcal{T}=\mathcal{T}_{\mathcal{F}}^{(i)}$. The group $\left(\operatorname{Sel}_{\left(\gamma^{2}-\kappa^{2}\left(\gamma^{\prime}\right) \gamma^{\prime}\right)}\right)^{\vee}$ has rank one or zero as an $\mathbb{H}_{\mathcal{F}}^{\text {ord }}$-module.

(2) Assume that $T_{0} \otimes \omega^{i^{\prime}}$ is isomorphic to its Kummer dual $\left(T_{0} \otimes \omega^{i^{\prime}}\right)^{*}(1)$ with certain $0 \leqslant i^{\prime} \leqslant p-2$, where $T_{0}:=\mathcal{T}_{\left(\gamma^{2}-\kappa^{2}\left(\gamma^{\prime}\right) \gamma^{\prime}\right)} /\left(\gamma^{\prime}-1\right) \mathcal{T}_{\left(\gamma^{2}-\kappa^{2}\left(\gamma^{\prime}\right) \gamma^{\prime}\right)}$. In this case, we have

$$
\operatorname{rank}_{\mathbb{H}_{\mathcal{F}} \operatorname{rrd}}\left(\operatorname{Sel}_{\left(\gamma^{2}-\kappa^{2}\left(\gamma^{\prime}\right) \gamma^{\prime}\right)}\right)^{\vee}= \begin{cases}1 & \text { if } \epsilon(l)=-1 \text { for every } l>0, \\ 0 & \text { if } \epsilon(l)=1 \text { for every } l>0,\end{cases}
$$

where $\epsilon(l)$ is the sign of the functional equation of $L$-function for a specialization of $\mathcal{F} \otimes \omega^{i^{\prime}-i}$ as explained in the following remark.

\section{Remark 7.12 .}

(1) Suppose that $T_{0} \otimes \omega^{i^{\prime}}$ is isomorphic to the Kummer dual of itself. For each $l$, we put $P_{l}=$ $\gamma^{\prime}-\kappa^{a(l)}\left(\gamma^{\prime}\right)$ for each integer $l>0$ with $a(l):=2\left(i^{\prime}-i\right)+2 l(p-1)$. For each $l>0, \mathcal{T} /\left(P_{l}\right) \mathcal{T}$ is isomorphic to the Tate-twist $T_{f_{l}}(a(l) / 2+1)$ of Deligne's Galois representation $T_{f_{l}}$ for an eigen-cuspform $f_{l}$ of weight $2+a(l)$. The sign $\epsilon(l)= \pm 1$ is the sign of the functional equation $\Lambda\left(f_{l}, s\right)=\epsilon(l) \Lambda\left(f_{l}, 2+a(l)-s\right)$ where $\Lambda\left(f_{l}, s\right)$ is the Hecke $L$-function for $f_{l}$ with its $\Gamma$-factor.

(2) The phenomena for the generic rank on the line $\left(\gamma^{2}-\kappa^{2}\left(\gamma^{\prime}\right) \gamma^{\prime}\right)$ was first studied and conjectured at least under the condition as in part (1) (see, for example, [NP00, §0]). We believe that such phenomena are always true even in the case without the functional equation.

Suppose that $\left(\operatorname{Sel}_{\left(\gamma^{2}-\kappa^{2}\left(\gamma^{\prime}\right) \gamma^{\prime}\right)}\right)^{\vee}$ is a torsion $\mathbb{H}_{\mathcal{F}}^{\text {ord }}$-module. We define $L_{p}\left(\mathcal{T}_{\left(\gamma^{2}-\kappa^{2}\left(\gamma^{\prime}\right) \gamma^{\prime}\right)}\right) \in \mathbb{H}_{\mathcal{F}}^{\text {ord }}$ to be the specialization of the two-variable $p$-adic $L$-function $L_{p}^{\mathrm{Ki}}(\mathcal{T}) \in \mathbb{H}_{\mathcal{F}}^{\text {n.o }}$ via $\mathbb{H}_{\mathcal{F}}^{\text {n.o }} \longrightarrow \mathbb{H}_{\mathcal{F}}^{\text {n.o }} /\left(\gamma^{2}-\right.$ $\left.\kappa^{2}\left(\gamma^{\prime}\right) \gamma^{\prime}\right) \cong \mathbb{H}_{\mathcal{F}}^{\text {ord }}$, in this case.

Conjecture 7.13. Suppose that $\left(\operatorname{Sel}_{\left(\gamma^{2}-\kappa^{2}\left(\gamma^{\prime}\right) \gamma^{\prime}\right)}\right)^{\vee}$ is a torsion $\mathbb{H}_{\mathcal{F}}^{\text {ord }}$-module. Then, we have the following equality:

$$
\operatorname{length}_{\left(\mathbb{H}_{\mathcal{F}}^{\text {ord }}\right)_{\mathfrak{l}}}\left(\operatorname{Sel}_{\left(\gamma^{2}-\kappa^{2}\left(\gamma^{\prime}\right) \gamma^{\prime}\right)}^{\vee}\right) \mathfrak{l}=\operatorname{ord}_{\mathfrak{l}}\left(L_{p}\left(\mathcal{T}_{(\gamma-\chi(\gamma))}\right)\right),
$$

for each height-one prime $\mathfrak{l}$ of $\mathbb{H}_{\mathcal{F}}^{\text {ord }}$.

Corollary 7.14. Suppose that $\left(\operatorname{Sel}_{\left(\gamma^{2}-\kappa^{2}\left(\gamma^{\prime}\right) \gamma^{\prime}\right)}\right)^{\vee}$ is a torsion $\mathbb{H}_{\mathcal{F}}^{\text {ord }}$-module.

(1) The two-variable IMC (Conjecture 2.4) implies Conjecture 7.13.

(2) Assume further the conditions listed in Theorem 2 of $\S 2$ with $P_{\tau}$ a unit in $\mathbb{H}_{\mathcal{F}}^{\text {n.o. }}$. Then Conjecture 7.13 implies the two-variable IMC (Conjecture 2.4).

Finally, in a general case where $\left(\operatorname{Sel}_{\left(\gamma^{2}-\kappa^{2}\left(\gamma^{\prime}\right) \gamma^{\prime}\right)}\right)^{\vee}$ is not necessarily a torsion $\mathbb{H}_{\mathcal{F}}^{\text {ord }}$-module, we propose the following IMC.

ConjeCture 7.15. Suppose that $\left(\operatorname{Sel}_{\left(\gamma^{2}-\kappa^{2}\left(\gamma^{\prime}\right) \gamma^{\prime}\right)}\right)^{\vee}$ is an $\mathbb{H}_{\mathcal{F}}^{\text {ord }}$-module with generic rank

$$
r=\operatorname{dim}_{\operatorname{Frac}\left(\mathbb{H}_{\mathcal{F}}^{\text {ord }}\right)}\left(\operatorname{Sel}_{\left(\gamma^{2}-\kappa^{2}\left(\gamma^{\prime}\right) \gamma^{\prime}\right)}\right)^{\vee} \otimes_{\mathbb{H}_{\mathcal{F}}^{\text {ord }}} \operatorname{Frac}\left(\mathbb{H}_{\mathcal{F}}^{\text {ord }}\right) .
$$

Let $\mathfrak{X}$ be the $\mathbb{H}_{\mathcal{F}}^{\text {ord }}$-torsion part of the $\mathbb{H}_{\mathcal{F}}^{\text {ord }}$-module $\left(\operatorname{Sel}_{\left(\gamma^{2}-\kappa^{2}\left(\gamma^{\prime}\right) \gamma^{\prime}\right)}\right)^{\vee}$. Then the following statements hold. 


\section{T. OCHIAI}

(1) The order $\operatorname{ord}_{\left(\gamma^{2}-\kappa^{2}\left(\gamma^{\prime}\right) \gamma^{\prime}\right)}\left(L_{p}(\mathcal{T})\right)$ is equal to $r$.

(2) For every height-one prime $\mathfrak{l}$ of $\mathbb{H}_{\mathcal{F}}^{\text {ord }}$, we have

$$
\operatorname{length}_{\left(\mathbb{H}_{\mathcal{F}}^{\text {ord }}\right)_{\mathfrak{l}}}(\mathfrak{X})_{\mathfrak{l}}=\operatorname{ord}_{\mathfrak{l}}\left(L_{p}\left(\mathcal{T}_{\left(\gamma^{2}-\kappa^{2}\left(\gamma^{\prime}\right) \gamma^{\prime}\right)}\right)\right) \text {, }
$$

where $L_{p}\left(\mathcal{T}_{\left(\gamma^{2}-\kappa^{2}\left(\gamma^{\prime}\right) \gamma^{\prime}\right)}\right)$ is defined to be the image of $L_{p}(\mathcal{T}) /\left(\gamma^{2}-\kappa^{2}\left(\gamma^{\prime}\right) \gamma^{\prime}\right)^{r}$ via $\mathbb{H}_{\mathcal{F}}^{\text {n.o }} \longrightarrow$ $\mathbb{H}_{\mathcal{F}}^{\text {n.o }} /\left(\gamma^{2}-\kappa^{2}\left(\gamma^{\prime}\right) \gamma^{\prime}\right) \cong \mathbb{H}_{\mathcal{F}}^{\text {ord }}$.

\section{Pseudo-null submodule}

In this section, we give a sufficient condition (Proposition 8.1) for $\left(\mathrm{Sel}_{\mathcal{T}}\right)^{\vee}$ to have no non-trivial pseudo-null submodule. Our proof relies on the method in Greenberg's paper [Gre87] (see also Remark 8.2). The result in this section is used in $\S 9$ in order to study examples where we can determine the structure of the Selmer group. In this section, we do not necessarily assume Condition (Ir). Instead of Condition (Ir), we will assume the following condition.

CONDition (Fr). $\mathbb{T}_{\mathcal{F}}^{\text {ord }}$ (respectively $\mathcal{T}_{\mathcal{F}}^{(i)}$ ) is free of rank two over $\mathbb{H}_{\mathcal{F}}^{\text {ord }}$ (respectively $\mathbb{H}_{\mathcal{F}}^{\text {n.o }}$ ).

As remarked in $\S 2$, Condition (Ir) implies Condition (Fr). Since we could find no reference for the pseudo-null submodule of the Selmer group for a Galois deformation, we decide to assume only a weaker condition (Fr) in this section for our later use. Our main proposition here is as follows.

Proposition 8.1. Let $\mathcal{T}=\mathcal{T}_{\mathcal{F}}^{(i)}$ be a nearly ordinary deformation satisfying Condition (Fr) and let $\Sigma$ be the set of ramified places for $\mathcal{T}$ (see $\S 2$ for the notation). Assume the following conditions:

(1) $\mathbb{H}_{\mathcal{F}}^{\text {n.o }}$ is a regular local ring;

(2) $\Sigma$ consists only of $\{p, \infty\}$.

Then $\left(\mathrm{Sel}_{\mathcal{T}}\right)^{\vee}$ has no non-trivial pseudo-null $\mathbb{H}_{\mathcal{F}}^{\text {n.o }}$-submodule.

Remark 8.2. Under similar assumptions, Greenberg [Gre87, Proposition 5] has proved that the Pontryagin dual of the Selmer group for the cyclotomic deformation of an ordinary $p$-adic representation $T \cong \mathbb{Z}_{p}^{d}$ has no pseudo-null $\mathbb{Z}_{p}[[\Gamma]]$-submodule when $T$ is unramified outside $\{p, \infty\}$. Our proof follows the idea of [Gre87, Proposition 5]. Since we treat the two-variable case, it is sometimes technically difficult to imitate his argument over the cyclotomic (one-variable) Iwasawa algebra, as is seen in our proof below.

Before giving the proof, we prepare several lemmas. Although our main proposition stated above only treats the case where $\Sigma$ consists only of $\{p, \infty\}$, we allow $\Sigma$ to contain primes other than $p$ in most of this section unless we state otherwise.

First, we prove the following lemma known as the weak Leopoldt conjecture for $\mathcal{T}$.

Lemma 8.3. We have $H^{2}\left(\mathbb{Q}_{\Sigma} / \mathbb{Q}, \mathcal{A}\right)=0$.

Proof. Note that $H^{2}\left(\mathbb{Q}_{\Sigma} / \mathbb{Q}, \mathcal{A}\right)$ is equal to the inductive limit $\lim _{s, t} H^{2}\left(\mathbb{Q}_{\Sigma} / \mathbb{Q}, A_{s, t}^{(j, k)}\right)$ for any pair $(j, k)$ with $1 \leqslant j \leqslant k-1$, where $A_{s, t}^{(j, k)}$ is the module defined in $\S 4.1$. From now on we assume further that $2 j \neq k$. It suffices to show that $H^{2}\left(\mathbb{Q}_{\Sigma} / \mathbb{Q}, A_{s, t}^{(j, k)}\right)=0$ for every $s, t$ under this condition. Since the Galois group $\operatorname{Gal}\left(\mathbb{Q}_{\Sigma} / \mathbb{Q}\right)$ has cohomological dimension $t w o, H^{3}\left(\mathbb{Q} \Sigma / \mathbb{Q}, A_{s, t}^{(j, k)}[p]\right)$ is zero. By the natural exact sequence

$H^{2}\left(\mathbb{Q}_{\Sigma} / \mathbb{Q}, A_{s, t}^{(j, k)}[p]\right) \longrightarrow H^{2}\left(\mathbb{Q}_{\Sigma} / \mathbb{Q}, A_{s, t}^{(j, k)}\right) \stackrel{\times p}{\longrightarrow} H^{2}\left(\mathbb{Q}_{\Sigma} / \mathbb{Q}, A_{s, t}^{(j, k)}\right) \longrightarrow H^{3}\left(\mathbb{Q}_{\Sigma} / \mathbb{Q}, A_{s, t}^{(j, k)}[p]\right) \longrightarrow \cdots$ 


\section{ON THE TWO-VARIABLE IWASAWA MAIN CONJECTURE}

$H^{2}\left(\mathbb{Q}_{\Sigma} / \mathbb{Q}, A_{s, t}^{(j, k)}\right)$ must be a $p$-divisible abelian group. On the other hand, by Kato's result $[$ Kat04, $\S 14], H^{2}\left(\mathbb{Q}_{\Sigma} / \mathbb{Q}, A_{s, t}^{(j, k)}\right)$ is finite for each $s, t$ under the above assumption on $(j, k)$. Hence, $H^{2}\left(\mathbb{Q}_{\Sigma} / \mathbb{Q}, A_{s, t}^{(j, k)}\right)$ must be zero.

Lemma 8.4. Assume that $\mathbb{H}_{\mathcal{F}}^{\text {n.o }}$ is regular and that $\mathbb{H}_{\mathcal{F}}^{\text {n.o }}$ satisfies Condition $(F r) . H^{1}(\mathbb{Q} \Sigma / \mathbb{Q}, \mathcal{A})^{\vee}$ has no non-trivial pseudo-null $\mathbb{H}_{\mathcal{F}}^{\text {n.o }}$-submodule.

Proof. Let $N$ be the largest pseudo-null submodule of $H^{1}\left(\mathbb{Q}_{\Sigma} / \mathbb{Q}, \mathcal{A}\right)^{\vee}$. Let $h$ be an arbitrary irreducible element of $\mathbb{H}_{\mathcal{F}}^{\text {n.o. }}$. By taking the short exact sequence:

$$
0 \longrightarrow \mathcal{A}[h] \longrightarrow \mathcal{A} \stackrel{\times h}{\longrightarrow} \mathcal{A} \longrightarrow 0,
$$

and by using Lemma 8.3, we have

$$
H^{1}\left(\mathbb{Q}_{\Sigma} / \mathbb{Q}, \mathcal{A}\right) /(h) H^{1}\left(\mathbb{Q}_{\Sigma} / \mathbb{Q}, \mathcal{A}\right) \cong H^{2}\left(\mathbb{Q}_{\Sigma} / \mathbb{Q}, \mathcal{A}[h]\right) .
$$

By a similar argument as that used in the proof of Lemma 8.3 depending on the Galois cohomological dimension of $\operatorname{Gal}\left(\mathbb{Q}_{\Sigma} / \mathbb{Q}\right), H^{2}\left(\mathbb{Q}_{\Sigma} / \mathbb{Q}, \mathcal{A}[h]\right)^{\vee}$ is shown to be torsion-free over the local domain $\mathbb{H}_{\mathcal{F}}^{\text {n.o }} /(h)$. Consequently, $H^{1}\left(\mathbb{Q}_{\Sigma} / \mathbb{Q}, \mathcal{A}\right)^{\vee}[h]$ must be a torsion-free $\mathbb{H}_{\mathcal{F}}^{\text {n.o }} /(h)$-module by taking the Pontryagin dual of (17). The torsion part $N[h]$ is also a torsion-free $\mathbb{H}_{\mathcal{F}}^{\text {n.o }} /(h)$-module since $N[h]$ is a sub $\mathbb{H}_{\mathcal{F}}^{\text {n.o }} /(h)$-module of $H^{1}\left(\mathbb{Q}_{\Sigma} / \mathbb{Q}, \mathcal{A}\right)^{\vee}[h]$. On the other hand, the torsion part $N[h]$ of $N$ for the height-one prime $(h)$ is a torsion $\mathbb{H}_{\mathcal{F}}^{\text {n.o }} /(h)$-module because $N$ is a pseudo-null $\mathbb{H}_{\mathcal{F}}^{\text {n.o }}$-module. Thus, $N[h]$ is zero for any irreducible element $h \in \mathbb{H}_{\mathcal{F}}^{\text {n.o. }}$. This completes the proof for $N=0$.

A finitely generated $\mathbb{H}_{\mathcal{F}}^{\text {n.o }}$-module $V$ is called reflexive if the canonical homomorphism $V \longrightarrow$ $V^{* *}:=\operatorname{Hom}_{\mathbb{H}_{\mathcal{F}}^{\text {n.o }}}\left(\operatorname{Hom}_{\mathbb{H}_{\mathcal{F}}^{\text {n.o }}}\left(V, \mathbb{H}_{\mathcal{F}}^{\text {n.o }}\right), \mathbb{H}_{\mathcal{F}}^{\text {n.o }}\right)$ is an isomorphism. We have the following lemma.

Lemma 8.5. Assume that $\mathbb{H}_{\mathcal{F}}^{\text {n.o }}$ is a regular local ring. Then, $H^{1}\left(\mathbb{Q}_{p}, \mathrm{~F}^{-} \mathcal{A}\right)^{\vee}$ is a reflexive $\mathbb{H}_{\mathcal{F}}^{\text {n.o }}$ module.

Proof. First, we show that $H^{1}\left(\mathbb{Q}_{p}, \mathrm{~F}^{-} \mathcal{A}\right)^{\vee}$ is torsion-free over $\mathbb{H}_{\mathcal{F}}^{\text {n.o. }}$. By taking the Pontryagin dual, it is equivalent to the statement that $H^{1}\left(\mathbb{Q}_{p}, \mathrm{~F}^{-} \mathcal{A}\right)$ is a divisible $\mathbb{H}_{\mathcal{F}}^{\text {n.o }}$-module. Consider the long exact sequence of the $G_{\mathbb{Q}_{p}}$-cohomology of

$$
0 \longrightarrow \mathrm{F}^{-} \mathcal{A}[h] \longrightarrow \mathrm{F}^{-} \mathcal{A} \stackrel{\times h}{\longrightarrow} \mathrm{F}^{-} \mathcal{A} \longrightarrow 0,
$$

for an irreducible element $h \in \mathbb{H}_{\mathcal{F}}^{\text {n.o }}, H^{1}\left(\mathbb{Q}_{p}, \mathrm{~F}^{-} \mathcal{A}\right) /(h) H^{1}\left(\mathbb{Q}_{p}, \mathrm{~F}^{-} \mathcal{A}\right)$ is a $\mathbb{H}_{\mathcal{F}}^{\text {n.o }}$-submodule of $H^{2}\left(\mathbb{Q}_{p}, \mathrm{~F}^{-} \mathcal{A}[h]\right)$. By the local Tate duality theorem, $H^{2}\left(\mathbb{Q}_{p}, \mathrm{~F}^{-} \mathcal{A}[h]\right)$ is the Pontryagin dual of $H^{0}\left(\mathbb{Q}_{p},\left(\mathrm{~F}^{-} \mathcal{A}(-1)\right)^{\vee} /(h)\left(\mathrm{F}^{-} \mathcal{A}(-1)\right)^{\vee}\right)$. This group must be zero, because $\left(\left(\mathrm{F}^{-} \mathcal{A}(-1)\right)^{\vee}\right)_{G_{\mathbb{Q}_{p}}}$ has support whose codimension is equal to or greater than two. Hence, $H^{1}\left(\mathbb{Q}_{p}, \mathrm{~F}^{-} \mathcal{A}\right)$ is a divisible $\mathbb{H}_{\mathcal{F}}^{\text {n.o }}$ module. Since the Pontryagin dual $H^{1}\left(\mathbb{Q}_{p}, \mathrm{~F}^{-} \mathcal{A}\right)^{\vee}$ has no non-trivial $\mathbb{H}_{\mathcal{F}}^{\text {n.o }}$-torsion submodule, the structure theorem of finitely generated $\mathbb{H}_{\mathcal{F}}^{\text {n.o }}$-modules (cf. Proposition 5.17 and Proposition 5.1.8 in [NSW00]) gives us an exact sequence

$$
0 \longrightarrow H^{1}\left(\mathbb{Q}_{p}, \mathrm{~F}^{-} \mathcal{A}\right)^{\vee} \longrightarrow V \longrightarrow Z \longrightarrow 0,
$$

where $V$ is a reflexive $\mathbb{H}_{\mathcal{F}}^{\text {n.o }}$-module and $Z$ is a pseudo-null $\mathbb{H}_{\mathcal{F}}^{\text {n.o }}$-module. Let $h^{\prime} \in \mathbb{H}_{\mathcal{F}}^{\text {n.o }}$ be an arbitrary non-zero irreducible element and let us consider the snake lemma in the following commutative diagram.

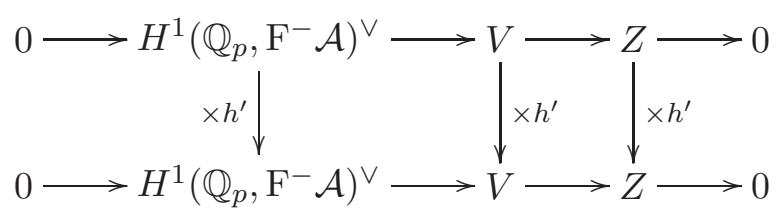




\section{T. OCHIAI}

By applying the snake lemma to the above diagram, we have an injection

$$
Z\left[h^{\prime}\right] \hookrightarrow\left(H^{1}\left(\mathbb{Q}_{p}, \mathrm{~F}^{-} \mathcal{A}\right)\left[h^{\prime}\right]^{\vee}\right)_{\mathbb{H}_{\mathcal{F}}^{\text {n.o }}} /\left(h^{\prime}\right) \text {-tor } .
$$

Since $H^{1}\left(\mathbb{Q}_{p}, \mathrm{~F}^{-} \mathcal{A}\right)\left[h^{\prime}\right]^{\vee}$ is naturally an $\mathbb{H}_{\mathcal{F}}^{\text {n.o }} /\left(h^{\prime}\right)$-submodule of $H^{1}\left(\mathbb{Q}_{p}, \mathrm{~F}^{-} \mathcal{A}\left[h^{\prime}\right]\right)^{\vee}$, this also gives us an injection

$$
Z\left[h^{\prime}\right] \hookrightarrow H^{1}\left(\mathbb{Q}_{p}, \mathrm{~F}^{-} \mathcal{A}\left[h^{\prime}\right]\right)_{\mathbb{H}_{\mathcal{F}}^{\text {n.o }}}^{\vee}\left(h^{\prime}\right) \text {-tor } .
$$

By a similar argument as above, we show that $H^{1}\left(\mathbb{Q}_{p}, \mathrm{~F}^{-} \mathcal{A}\left[h^{\prime}\right]\right)^{\vee}$ is a torsion-free $\mathbb{H}_{\mathcal{F}}^{\text {n.o }} /\left(h^{\prime}\right)$-module by condition (2) of the lemma. Hence, $Z$ must be zero. This completes the proof.

Lemma 8.6. Assume the three conditions stated in Proposition 8.1. Then, we have the following exact sequence:

$$
0 \longrightarrow \operatorname{Sel}_{\mathcal{T}} \longrightarrow H^{1}\left(\mathbb{Q}_{\{p, \infty\}} / \mathbb{Q}, \mathcal{A}\right) \stackrel{\text { loc }}{\longrightarrow} H^{1}\left(\mathbb{Q}_{p}, \mathrm{~F}^{-} \mathcal{A}\right) \longrightarrow 0 .
$$

Proof. By Corollary 4.12, we have the following exact sequence:

$$
0 \longrightarrow \operatorname{Sel}_{\mathcal{T}} \longrightarrow H^{1}\left(\mathbb{Q}_{\{p, \infty\}} / \mathbb{Q}, \mathcal{A}\right) \stackrel{\text { loc }}{\longrightarrow} \frac{H^{1}\left(\mathbb{Q}_{p}, \mathcal{A}\right)}{H_{\mathrm{Gr}}^{1}\left(\mathbb{Q}_{p}, \mathcal{A}\right)} \longrightarrow 0
$$

Since the restriction map $H^{1}\left(\mathbb{Q}_{p}, \mathrm{~F}^{-} \mathcal{A}\right) \longrightarrow H^{1}\left(I_{p}, \mathrm{~F}^{-} \mathcal{A}\right)^{G_{\mathbb{Q} p}}$ is an isomorphism, we have an exact sequence

$$
0 \longrightarrow \frac{H^{1}\left(\mathbb{Q}_{p}, \mathcal{A}\right)}{H_{\mathrm{Gr}}^{1}\left(\mathbb{Q}_{p}, \mathcal{A}\right)} \longrightarrow H^{1}\left(\mathbb{Q}_{p}, \mathrm{~F}^{-} \mathcal{A}\right) \longrightarrow H^{2}\left(\mathbb{Q}_{p}, \mathrm{~F}^{+} \mathcal{A}\right) .
$$

Note that $H^{2}\left(\mathbb{Q}_{p}, \mathrm{~F}^{+} \mathcal{A}\right)$ is zero by the same argument as the proof of Claim 4.8. This completes the proof of the lemma.

Lemma 8.7. Let $R$ be a Noetherian complete regular local ring and let $M$ be an $R$-module which has the following presentation

$$
0 \longrightarrow W \longrightarrow U \longrightarrow M \longrightarrow 0,
$$

where $U$ is a finitely generated $R$-module which has no non-trivial pseudo-null $R$-submodule and $W$ is a reflexive $R$-module. Then $M$ has no non-trivial pseudo-null $R$-submodule.

Proof. Suppose that the largest pseudo-null $R$-submodule $M_{\text {null }}$ of $M$ is non-trivial. We denote by $U_{0} \subset U$ the inverse image of $M_{\text {null }}$ via the natural projection $U \rightarrow M$. Since $U$ has no non-trivial pseudo-null $R$-submodule, $U_{0}$ also has no non-trivial pseudo-null $R$-submodule. By the structure theorem of finitely generated $R$-modules, we have the following exact sequence:

$$
0 \longrightarrow U_{0} \longrightarrow E \oplus W^{\prime} \longrightarrow Z \longrightarrow 0,
$$

where $E$ is an elementary torsion $R$-module, $W^{\prime}$ is a reflexive $R$-module and $Z$ is a pseudo-null $R$-module. Thus, we also have the following exact sequence:

$$
0 \longrightarrow W \longrightarrow E \oplus W^{\prime} \longrightarrow Z^{\prime} \longrightarrow 0
$$

where $Z^{\prime}$ is an extension of $Z$ by $M_{\text {null }}$. In particular, $Z^{\prime}$ is a non-trivial pseudo-null $R$-module. Since $W$ is reflexive and $Z^{\prime}$ is pseudo-null, $E$ must be trivial. Thus, we have an injection $W \hookrightarrow$ $W^{\prime}$ whose cokernel is a non-trivial pseudo-null $R$-module. Note that $\operatorname{Ext}_{R}^{1}\left(W^{\prime} / W, R\right)$ is zero since $W^{\prime} / W$ is pseudo-null (see [OV02, Proposition 3.4] for example). The injection $W \hookrightarrow W^{\prime}$ induces an isomorphism $\operatorname{Hom}_{R}\left(W^{\prime}, R\right) \stackrel{\sim}{\longrightarrow} \operatorname{Hom}_{R}(W, R)$. Hence, $W \hookrightarrow W^{\prime}$ must be an isomorphism since $W$ and $W^{\prime}$ are reflexive $R$-modules. This contradicts the assumption that $M_{\text {null }}$ is non-trivial. The proof is complete.

Finally we give the proof of Proposition 8.1. 


\section{ON THE TWO-VARIABLE IWASAWA MAIN CONJECTURE}

Proof of Proposition 8.1. By Lemma 8.6, we have the following exact sequence:

$$
0 \longrightarrow H^{1}\left(\mathbb{Q}_{p}, \mathrm{~F}^{-} \mathcal{A}\right)^{\vee} \longrightarrow H^{1}\left(\mathbb{Q}_{\{p, \infty\}} / \mathbb{Q}, \mathrm{F}^{-} \mathcal{A}\right)^{\vee} \longrightarrow\left(\operatorname{Sel}_{\mathcal{T}}\right)^{\vee} \longrightarrow 0 .
$$

The module $H^{1}\left(\mathbb{Q}_{p}, \mathrm{~F}^{-} \mathcal{A}\right)^{\vee}$ is reflexive over $\mathbb{H}_{\mathcal{F}}^{\text {n.o }}$ by Lemma 8.5. The $\mathbb{H}_{\mathcal{F}}^{\text {n.o }}$-module $H^{1}\left(\mathbb{Q}_{\{p, \infty\}} / \mathbb{Q}\right.$, $\left.\mathrm{F}^{-} \mathcal{A}\right)^{\vee}$ has no non-trivial pseudo-null $\mathbb{H}_{\mathcal{F}}^{\text {n.o }}$-submodule by Lemma 8.4. Thus, we complete the proof by applying Lemma 8.7 .

\section{Examples}

In this section, we study examples of two-variable nearly ordinary deformations where we can determine the structure of the Selmer group or we prove the equality in addition to the inequality result proved by using Beilinson-Kato elements.

First, we prepare some preliminary results.

Proposition 9.1 [Gre87, Theorem 2]. Let $T$ be a $\operatorname{Gal}\left(\mathbb{Q}_{\Sigma} / \mathbb{Q}\right)$-module which is free of finite rank over $\mathbb{Z}_{p}$. Suppose that $T$ is ordinary and critical at $p$. Then we have

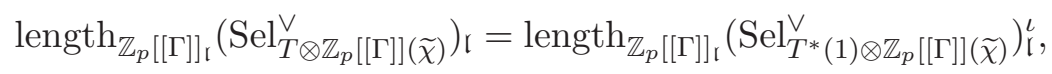

for every height-one prime $\mathfrak{l}$ in $\mathbb{Z}_{p}[[\Gamma]]_{\mathfrak{r}}$ where $\iota$ is the canonical involution of $\mathbb{Z}_{p}[[\Gamma]]$ induced by $g \mapsto g^{-1}$ for $g \in \Gamma$.

We recall the following lemma.

Lemma 9.2. Let $R$ be a Noetherian complete regular local ring of Krull dimension $n \geqslant 2$ and let $N$ be a pseudo-null $R$-module. Let $I$ be a height-one prime of $R$ such that $R / I$ is a regular local ring of Krull dimension $n-1$. Then, we have the following equality for every height-one prime ideal in $R / I$ :

$$
\operatorname{length}_{(R / I)_{\mathfrak{l}}}\left(N[I]_{\mathfrak{l}}\right)=\operatorname{length}_{(R / I)_{\mathfrak{l}}}(N / I N)_{\mathfrak{l}} \text {. }
$$

In particular, $N[I]$ is a pseudo-null $R / I$-module if and only if $N / I N$ is a pseudo-null $R / I$-module.

Although this lemma might be known to the experts, we refer the reader to [Och05, Lemma 3.1] for the proof if necessary.

Lemma 9.3. Let $R$ be a Noetherian complete regular local ring of Krull dimension greater than or equal to two. Let $M$ (respectively $N$ ) be a torsion $R$-module $R /(f)$ (respectively $R /(g)$ ) with $f \in R$ (respectively $g \in R$ ). Suppose that we have a family $\left\{J_{l}\right\}_{1 \leqslant l<\infty}$ of non-zero elements of $R$ satisfying the following properties.

(1) We have an injection $M \hookrightarrow \prod_{1 \leqslant l<\infty} M / J_{l} M$.

(2) For each $i, R / J_{l}$ is a regular local ring.

(3) The modules $M / J_{l} M$ and $N / J_{l} N$ are torsion over $R / J_{l}$.

(4) We have $\operatorname{char}_{R / J_{l}}\left(M / J_{l} M\right) \supset \operatorname{char}_{R / J_{l}}\left(N / J_{l} N\right)$ for each $l \geqslant 1$.

Then, we have $\operatorname{char}_{R}(M) \supset \operatorname{char}_{R}(N)$.

Proof. It suffices to show that the image of $g$ via $R \rightarrow M$ is zero. By conditions (3) and (4), the image of $g$ via $R \rightarrow M / J_{l} M$ is zero for any $l$. This completes the proof by condition (1).

\subsection{IMC for Ramanujan's cuspform}

Let $\Delta \in S_{12}\left(\mathrm{SL}_{2}(\mathbb{Z})\right)$ be the unique eigen-cuspform of level 1 and weight 12 , whose $q$-expansion is equal to $q \prod_{1 \leqslant n<\infty}\left(1-q^{n}\right)^{24}$. The only known non-ordinary primes for $\Delta$ are $p=2,3,5,7,2411$ 


\section{T. OCHIAI}

at the moment. For all other primes $p$, we have the ordinary $\Lambda$-adic newform $\mathcal{F}(\Delta) \in \mathbb{Z}_{p}\left[\left[\Gamma^{\prime}\right]\right][[q]]$ such that the specialization of $\mathcal{F}$ under $\mathbb{Z}_{p}\left[\left[\Gamma^{\prime}\right]\right][[q]] \longrightarrow \mathbb{Z}_{p}[[q]], \gamma^{\prime} \mapsto \kappa^{10}\left(\gamma^{\prime}\right)$ coincides with the $q$-expansion of the $p$-stabilization $\Delta^{(p)}$ of $\Delta$ (we omit the prime $p$ in the notation $\mathcal{F}(\Delta)$ unless there is a possibility of confusion). See [Hid93, $\S 7.6]$ for the explanation on the Hida family for $\Delta$. Condition (Nor) is always satisfied. The two-variable Iwasawa theory for $\mathcal{T}=\mathcal{T}_{\mathcal{F}(\Delta)}^{(i)}$ at $p$ is of our interest.

Question 9.4. For which ordinary prime $p$ of $\Delta$ and for which integer $i$ with $0 \leqslant i \leqslant p-2$ is the characteristic ideal $\operatorname{char}_{\mathbb{H}_{\mathcal{F}}^{\text {noo }}}\left(\operatorname{Sel}_{\mathcal{T}_{\mathcal{F}(\Delta)}^{(i)}}\right)^{\vee}$ or the ideal $\left(L_{p}\left(\mathcal{T}_{\mathcal{F}(\Delta)}^{(i)}\right)\right)$ non-trivial?

Recall that the value $L(\Delta, j) /(2 \pi \sqrt{-1})^{j} \Omega_{\infty, \Delta}^{(-1)^{j-1}}$ is equal to $2^{3} \cdot 3^{4} \cdot 5 \cdot 7 / 691,2^{4} \cdot 3,2 \cdot 7,5^{2}, 3^{2}, 2^{2} \cdot 5$ when $j=1, \ldots, 6$. We have

$$
\frac{L(\Delta, j)}{(2 \pi \sqrt{-1})^{j} \Omega_{\infty, \Delta}^{(-1)^{j-1}}}=-\frac{L(\Delta, 12-j)}{(2 \pi \sqrt{-1})^{j} \Omega_{\infty, \Delta}^{(-1)^{11-j}}}
$$

by the functional equation. In particular, the value $L(\Delta, j) /(2 \pi \sqrt{-1})^{j} \Omega_{\infty, \Delta}^{(-1)^{j-1}}$ is a $p$-adic unit for every $j$ with $1 \leqslant j \leqslant 11$ and for $p \geqslant 11$ with $p \neq 691$.

Let $p$ be an ordinary prime of $\Delta$ where Condition (Ir) is satisfied (in particular, $p \neq 691$ ). For $1 \leqslant i \leqslant 11$, we have

$$
\begin{aligned}
\left(\chi^{i} \circ \kappa^{10}\right) L_{p}^{\mathrm{Ki}}\left(\mathcal{T}_{\mathcal{F}(\Delta)}^{(i)}\right) & =\left(1-\frac{p^{i-1}}{a_{p}\left(\Delta^{(p)}\right)}\right) \frac{L\left(\Delta^{(p)}, i\right)}{(2 \pi \sqrt{-1})^{i-1} \Omega_{\infty, \Delta}^{(-1)^{i-1}}} \\
& =\left(1-\frac{p^{i-1}}{a_{p}\left(\Delta^{(p)}\right)}\right)\left(1-\frac{a_{p}(\Delta)}{p^{i}}\right) \frac{L(\Delta, i)}{(2 \pi \sqrt{-1})^{i-1} \Omega_{\infty, \Delta}^{(-1)^{i-1}}} .
\end{aligned}
$$

For $i \neq 1$, this is a $p$-adic unit, hence we have $L_{p}^{\mathrm{Ki}}\left(\mathcal{T}_{\mathcal{F}(\Delta)}^{(i)}\right) \in \mathbb{Z}_{p}\left[\left[\Gamma \times \Gamma^{\prime}\right]\right]^{\times}$. For $i=1, L_{p}^{\mathrm{Ki}}\left(\mathcal{T}_{\mathcal{F}(\Delta)}^{(1)}\right)$ is a unit if and only if $a_{p}(\Delta) \not \equiv 1$ modulo $p$. As for the structure of the Selmer group, we have the following result.

Lemma 9.5. The Selmer group $\left(\operatorname{Sel}_{\mathcal{T}}\right)^{\vee}$ has no non-trivial pseudo-null $\Lambda^{(2)}$-submodule for $\mathcal{T}=$ $\mathcal{T}_{\mathcal{F}(\Delta)}^{(i)}$.

Proof. It suffices to see that our nearly ordinary deformation $\mathcal{T}$ associated to $\Delta$ satisfies the two conditions in Proposition 8.1. Condition (1) is deduced by observing the dimension of the space of weight 12 cuspforms (cf. [Hid93, $\S 7.6]$ ). Condition (2) is clear since $\Delta$ has level one. This completes the proof.

We summarize our argument above in the following proposition.

Proposition 9.6. Let $p$ be an ordinary prime of $\Delta$ where Condition (Ir) is satisfied.

(1) When $2 \leqslant i \leqslant 11, L_{p}^{\mathrm{Ki}}\left(\mathcal{T}_{\mathcal{F}(\Delta)}^{(i)}\right)$ is trivial and $\operatorname{Sel}_{\mathcal{T}_{\mathcal{F}(\Delta)}^{(i)}}=0$.

(2) When $i=1, L_{p}^{\mathrm{Ki}}\left(\mathcal{T}_{\mathcal{F}(\Delta)}^{(1)}\right)$ is non-trivial if and only if $a_{p}(\Delta) \equiv 1$ modulo $p$.

Remark 9.7.

(1) For $i=0$ or for $12 \leqslant i \leqslant p-2$, we do not have a precise conjecture about when or how often $L_{p}^{\mathrm{Ki}}\left(\mathcal{T}_{\mathcal{F}(\Delta)}^{(i)}\right)$ is non-trivial.

(2) The primes where $a_{p}(\Delta) \equiv 1$ modulo $p$ are called anomalous primes for $\Delta$. Among smaller primes, $p=11$ and 23 are known to be anomalous. We do not know how many other anomalous primes for $\Delta$ exist. 


\section{ON THE TWO-VARIABLE IWASAWA MAIN CONJECTURE}

According to the above remark, we will investigate the case $p=11$ in the next subsection.

\subsection{Ramanujan's cuspform at $p=11$}

In this subsection, we discuss the two-variable Iwasawa theory for $\mathcal{F}(\Delta)$ at $p=11$, where we have a Hida family $\mathcal{T}=\mathcal{T}_{\mathcal{F}(\Delta)}^{(1)} \cong \mathbb{Z}_{p}\left[\left[\Gamma \times \Gamma^{\prime}\right]\right]^{\oplus 2}$ such that:

(1) the specialization $\mathcal{T} / \Phi^{(1,2)} \mathcal{T}$ is isomorphic to the $p$-Tate module of $X_{0}(11)$;

(2) $\mathcal{T} / \Phi^{(j, 12)} \mathcal{T}$ is a lattice of the representation $T_{\Delta}(j) \otimes \omega^{1-j}$, where $T_{\Delta} \cong \mathbb{Z}_{p}^{\oplus 2}$ is the $p$-adic Galois representation associated to $\Delta$ by Deligne.

From now on, we shall denote $\mathbb{Z}_{p}\left[\left[\Gamma \times \Gamma^{\prime}\right]\right]$ by $\Lambda^{(2)}$ for short. Our results on the Iwasawa theory for $\mathcal{T}$ in this section are as follows.

RESUlts ON THE IWASAWA THEORY FOR $\mathcal{T}$. Let $\mathcal{T}=\mathcal{T}_{\mathcal{F}(\Delta)}^{(1)}$ with $p=11$.

(1) We have length $\Lambda_{\mathfrak{l}}^{(2)}\left(\operatorname{Sel}_{\mathcal{T}}^{\vee}\right)_{\mathfrak{l}}=\operatorname{ord}_{\mathfrak{l}}\left(L_{p}^{\mathrm{Ki}}(\mathcal{T})\right)$ for every height-one prime $\mathfrak{l}$ in $\Lambda^{(2)}$.

(2) We have $\left(\operatorname{Sel}_{\mathcal{T}}\right)^{\vee} \cong \Lambda^{(2)} /\left(\gamma^{2}-\kappa^{2}\left(\gamma^{\prime}\right) \gamma^{\prime}\right)$.

We will show statement (2) first and the equality in statement (1) will be proved later. Let us take an infinite family of elements $\left\{P_{l} \in \Lambda^{(2)}\right\}_{1 \leqslant l<\infty}$ given by $P_{l}=\gamma^{\prime}-\kappa^{2 l(p-1)}\left(\gamma^{\prime}\right)$. Then we have the following claim.

Claim 9.8. Let us denote by $T_{l}$ the representation associated to the ordinary eigen-cuspform $f_{2+2 l(p-1)}$ of weight $2+2 l(p-1)$ in the Hida family for $\Delta$.

(i) $\mathcal{T} /\left(P_{l}\right) \mathcal{T}$ is the cyclotomic deformation of $T_{l} \otimes \omega$.

(ii) The natural restriction map $\left(\operatorname{Sel}_{\mathcal{T}}\right)^{\vee} /\left(P_{l}\right)\left(\operatorname{Sel}_{\mathcal{T}}\right)^{\vee} \longrightarrow \operatorname{Sel}_{\mathcal{T} /\left(P_{l}\right) \mathcal{T}}$ is an isomorphism.

(iii) We have the isomorphism $\operatorname{Sel}_{\mathcal{T} /\left(P_{l}\right) \mathcal{T}} \cong \mathbb{Z}_{p}[[\Gamma]] /\left(\gamma-\chi^{1+l(p-1)}(\gamma)\right)$.

Proof. Statement (1) is nothing but the definition of $\mathcal{T}$. We have $H^{0}(\mathbb{Q}, \mathcal{A})=0$ by $[$ Ser72, 5.5.2] and by an argument using Nakayama's lemma (cf. the proof of Claim 9.11), where $\mathcal{A}=\mathcal{T} \otimes_{\Lambda^{(2)}}$ $\operatorname{Hom}_{\mathbb{Z}_{p}}\left(\Lambda^{(2)}, \mathbb{Q}_{p} / \mathbb{Z}_{p}\right)$. By definition, the set of ramified primes $\Sigma$ for $\mathcal{T}$ is $\{p, \infty\}$. Hence, statement (2) is a corollary of Proposition 5.2. Let us show the statement (3) in the remainder. For any $l \geqslant 0, \operatorname{Sel}_{\mathcal{T} /\left(P_{l}, p\right) \mathcal{T}}$ is isomorphic to $\operatorname{Sel}_{\mathcal{T} /\left(P_{0}, p\right) \mathcal{T}}$ by the congruence property. On the other hand, $\mathcal{T} /\left(P_{0}, p\right) \mathcal{T}$ is the cyclotomic deformation $X_{0}(11)[11] \otimes_{\mathbb{Z}_{p}} \mathbb{Z}_{p}[[\Gamma]](\tilde{\chi})$ of the group of 11-torsion elements $X_{0}(11)[11]$ of the modular elliptic curve $X_{0}(11)$. Hence, by [Gre91], $\operatorname{Sel}_{\mathcal{T} /\left(P_{0}, p\right) \mathcal{T}}$ is isomorphic to $\mathbb{Z}_{p}[[\Gamma]] /(\gamma-1, p) \cong \mathbb{Z} / p \mathbb{Z}$. By the control theorem for modulo- $(p)$ reduction which can be proved in the same manner as those in $\S 5$, we have an isomorphism $\left(\operatorname{Sel}_{\mathcal{T} /\left(P_{l}\right) \mathcal{T}}\right)^{\vee} /(p)\left(\operatorname{Sel}_{\mathcal{T} /\left(P_{l}\right) \mathcal{T}}\right)^{\vee} \cong$ $\left(\operatorname{Sel}_{\mathcal{T} /\left(P_{l}, p\right) \mathcal{T}}\right)^{\vee} \cong \mathbb{Z} / p \mathbb{Z}$. Since $\left(\operatorname{Sel}_{\mathcal{T} /\left(P_{l}\right) \mathcal{T}}\right)^{\vee}$ has no finite $\mathbb{Z}_{p}[[\Gamma]]$-submodule (cf. [Gre94, Proposition $10]),\left(\operatorname{Sel}_{\mathcal{T} /\left(P_{l}\right) \mathcal{T}}\right)^{\vee}$ must be a free $\mathbb{Z}_{p}$-module of rank one for any $l \geqslant 0$. Finally, let us denote the

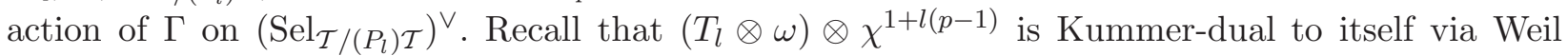
pairing. By Proposition 9.1 and statement $(1),\left(\operatorname{Sel}_{\mathcal{T} /\left(P_{l}\right) \mathcal{T}}\right)^{\vee} \otimes \chi^{-1-l(p-1)} \cong\left(\operatorname{Sel}_{\left(\mathcal{T} /\left(P_{l}\right) \mathcal{T}\right) \otimes \chi^{1+l(p-1)}}\right)^{\vee}$ is a free rank-one $\mathbb{Z}_{p}$-module with trivial $\Gamma$-action. Hence, we have $\left(\operatorname{Sel}_{\mathcal{T} /\left(P_{l}\right) \mathcal{T}}\right)^{\vee} \cong \mathbb{Z}_{p}\left(\chi^{1+l(p-1)}\right)$ for every $l \geqslant 1$, where $\mathbb{Z}_{p}\left(\chi^{1+l(p-1)}\right)$ is a free rank-one $\mathbb{Z}_{p}$-module on which $\Gamma$ acts via $\chi^{1+l(p-1)}$. This completes the proof of statement (3) and hence Claim 9.8 .

Let us prove the following claim.

Claim 9.9. We have length $\Lambda_{\mathfrak{l}}^{(2)}\left(\operatorname{Sel}_{\mathcal{T}}^{\vee}\right)_{\mathfrak{l}} \leqslant \operatorname{ord}_{\mathfrak{l}}\left(\gamma^{2}-\kappa^{2}\left(\gamma^{\prime}\right) \gamma^{\prime}\right)$ for every height-one prime $\mathfrak{l}$ in $\Lambda^{(2)}$.

Proof. Let $g$ be an element of $\Lambda^{(2)}$ such that $(g)=\operatorname{char}_{\Lambda^{(2)}}\left(\operatorname{Sel}_{\mathcal{T}}\right)^{\vee}$. Since $\left(\operatorname{Sel}_{\mathcal{T}}\right)^{\vee}$ has no non-trivial pseudo-null $\Lambda^{(2)}$-submodule, we have an injection $\left(\operatorname{Sel}_{\mathcal{T}}\right)^{\vee} \hookrightarrow \Lambda^{(2)} /(g)$ with a pseudo-null cokernel. 


\section{T. OCHIAI}

We may replace $\left(\operatorname{Sel}_{\mathcal{T}}\right)^{\vee}$ by $\Lambda^{(2)} /(g)$ to prove the claim. Let us apply Lemma 9.3 for $R=\Lambda^{(2)}$, $M=\Lambda^{(2)} /\left(\gamma^{2}-\kappa^{2}\left(\gamma^{\prime}\right) \gamma^{\prime}\right)$ and $N=\Lambda^{(2)} /(g)$. Let $P_{l}=\gamma^{\prime}-\kappa^{2 l(p-1)}\left(\gamma^{\prime}\right) \in \Lambda^{(2)}$ as above. Note that each $P_{l}$ is contained in $\mathbb{Z}_{p}\left[\left[\Gamma^{\prime}\right]\right]$. Since $P_{l}$ are relatively prime to each other, we have an injection

$$
\mathbb{Z}_{p}\left[\left[\Gamma^{\prime}\right]\right] \hookrightarrow \prod_{1 \leqslant l<\infty} \mathbb{Z}_{p}\left[\left[\Gamma^{\prime}\right]\right] /\left(P_{l}\right)
$$

On the other hand, $M$ is finite flat of degree two over $\mathbb{Z}_{p}\left[\left[\Gamma^{\prime}\right]\right]$. Hence, by applying the base extension $\otimes_{\mathbb{Z}_{p}\left[\left[\Gamma^{\prime}\right]\right]} M$ to (18), we have an injection $M \hookrightarrow \prod_{1 \leqslant l<\infty} M /\left(P_{l}\right) M$. Thus, we have shown condition (1) of Lemma 9.3. Condition 2 is satisfied since $P_{l}$ are polynomials of degree one.

Claim 9.10. We have length $\Lambda_{\mathfrak{l}}^{(2)}\left(\operatorname{Sel}_{\mathcal{T}}^{\vee}\right)_{\mathfrak{l}}=\operatorname{ord}_{\mathfrak{l}}\left(\gamma^{2}-\kappa^{2}\left(\gamma^{\prime}\right) \gamma^{\prime}\right)$ for every height-one prime $\mathfrak{l}$ in $\Lambda^{(2)}$.

Proof. Let us consider the specialization at $k=2$. The image of the two ideals $\left(\gamma^{2}-\kappa^{2}\left(\gamma^{\prime}\right) \gamma^{\prime}\right) \subset \Lambda^{(2)}$ and $(g) \subset \Lambda^{(2)}$ in $\Lambda^{(2)} /\left(\gamma^{\prime}-1\right)=\mathbb{Z}_{p}[[\Gamma]]$ are both equal to $(\gamma-1)$.

Hence, $\left(\operatorname{Sel}_{\mathcal{T}}\right)^{\vee}$ is a torsion $\Lambda^{(2)}$-module whose characteristic ideal is $\left(\gamma^{2}-\kappa^{2}\left(\gamma^{\prime}\right) \gamma^{\prime}\right)$. By Greenberg,

$$
\left(\operatorname{Sel}_{\mathcal{T}}\right)^{\vee} /\left(P_{0}\right)\left(\operatorname{Sel}_{\mathcal{T}}\right)^{\vee} \cong\left(\operatorname{Sel}_{T_{f_{2}} \otimes \mathbb{Z}_{p}[[\Gamma]](\widetilde{\chi})}\right)^{\vee}
$$

is isomorphic to $\mathbb{Z}_{p}$. In particular, $\left(\operatorname{Sel}_{\mathcal{T}}\right)^{\vee} /\left(P_{0}\right)\left(\operatorname{Sel}_{\mathcal{T}}\right)^{\vee}$ is a cyclic module over $\mathbb{Z}_{p}[[\Gamma]] \cong \mathbb{Z}_{p}[[\Gamma \times$ $\left.\left.\Gamma^{\prime}\right]\right] /\left(P_{0}\right)$. By Nakayama's lemma, $\left(\operatorname{Sel}_{\mathcal{T}}\right)^{\vee}$ has to be a cyclic module over $\mathbb{Z}_{p}\left[\left[\Gamma \times \Gamma^{\prime}\right]\right]$. Consequently, we have $\left(\operatorname{Sel}_{\mathcal{T}}\right)^{\vee} \cong \Lambda^{(2)} /\left(\gamma^{2}-\kappa^{2}\left(\gamma^{\prime}\right) \gamma^{\prime}\right)$.

Next, we shall study IMC for this $\mathcal{T}$. Theorems 1 and Theorem 2 given in $\S 2$ imply the following claim.

CLAIM 9.11. We have $\operatorname{length}_{\Lambda_{\mathfrak{l}}^{(2)}}\left(\operatorname{Sel}_{\mathcal{T}}^{\vee}\right)_{\mathfrak{l}} \leqslant \operatorname{ord}_{\mathfrak{l}}\left(L_{p}^{\mathrm{Ki}}(\mathcal{T})\right)$ for every height-one prime $\mathfrak{l}$ in $\Lambda^{(2)}$.

Proof. We shall check conditions (i) and (ii) of Theorem 2 for $\mathcal{T}$. By the nearly ordinary condition of $\mathcal{T}$, the image of $G_{\mathbb{Q}_{p}^{u r}\left(\mu_{p} \infty\right)}$ is contained in the group $\left\{\left(\begin{array}{ll}1 & * \\ 0 & 1\end{array}\right) \in \mathrm{GL}_{2}\left(\Lambda^{(2)}\right)\right\}$. Let us consider also the action of $G_{\mathbb{Q}_{p}^{u r}\left(\mu_{p} \infty\right)}$ on $\mathcal{T} / \mathfrak{M T} \cong \mathbb{F}_{p}^{\oplus 2}$ which is contained in the group $\left\{\left(\begin{array}{ll}1 & * \\ 0 & 1\end{array}\right) \in \mathrm{GL}_{2}\left(\mathbb{F}_{p}\right)\right\}$. Recall that the residual representation $\mathcal{T} / \mathfrak{M T}$ is isomorphic to the group of 11-torsion points of $X_{0}(11)$ by properties of $\mathcal{T}$ introduced at the beginning of $\S 9.2$. Since $X_{0}(11)$ has split multiplicative reduction at 11 , we have a $G_{\mathbb{Q}_{p}}$-equivariant isomorphism $X_{0}(11)\left(\overline{\mathbb{Q}}_{p}\right) \cong \overline{\mathbb{Q}}_{p}^{\times} / q^{\mathbb{Z}}$ with $q \in p \mathbb{Z}_{p}$ by the uniformization theory by Tate. Hence, we have

$$
0 \longrightarrow \mu_{p} \longrightarrow \mathcal{T} / \mathfrak{M T} \longrightarrow q^{\mathbb{Z}} / q^{\frac{1}{p} \mathbb{Z}} \longrightarrow 0 .
$$

We find $\tau \in G_{\mathbb{Q}_{p}^{\text {ur }}\left(\mu_{p} \infty\right)}$ such that the image $\left(\begin{array}{cc}1 & p_{\tau} \\ 0 & 1\end{array}\right)$ of $\tau$ in $\operatorname{Aut}(\mathcal{T} / \mathfrak{M T}) \cong \mathrm{GL}_{2}\left(\mathbb{F}_{p}\right)$ satisfies $p_{\tau} \neq 0$ because $q=11^{5} u$ with $u \in \mathbb{Z}_{11}^{\times}$. Thus, $\tau$ is presented as $\left(\begin{array}{cc}1 & P_{\tau} \\ 0 & 1\end{array}\right)$ under certain choice of basis $\operatorname{Aut}(\mathcal{T}) \cong \mathrm{GL}_{2}\left(\Lambda^{(2)}\right)$, where $P_{\tau}$ is a unit of $\Lambda^{(2)}$.

For condition (ii), $G_{\mathbb{Q}} \longrightarrow \operatorname{Aut}(\mathcal{T} / \mathfrak{M T})$ contains an element $\left(\begin{array}{cc}-1 & 0 \\ 0 & -1\end{array}\right)$ by the surjectivity of the representation of $G_{\mathbb{Q}}$ on the group of 11 -torsion points of $X_{0}(11)$ shown in [Ser72, 5.5.2]. This completes the proof by Theorem 2 .

Since we already have an inequality as in Claim 9.11, it suffices to see that $\operatorname{char}_{\Lambda^{(2)}}\left(\operatorname{Sel}_{\mathcal{T}}\right)^{\vee}$ modulo $\left(\gamma^{\prime}-1\right)$ is equal to the ideal of $\mathbb{Z}_{p}[[\Gamma]]$ generated by $L_{p}(\mathcal{T})$ modulo $\gamma^{\prime}-1$. By Claim 9.10, the ideal $\operatorname{char}_{\Lambda^{(2)}}\left(\operatorname{Sel}_{\mathcal{T}}\right)^{\vee}$ modulo $\left(\gamma^{\prime}-1\right)$ of $\mathbb{Z}_{p}[[\Gamma]]$ is equal to $\left(\gamma^{2}-1\right)=(\gamma-1)$ (note that $\gamma+1$ is a unit in $\left.\mathbb{Z}_{p}[[\Gamma]]\right)$. On the other hand, by the interpolation property given in Theorem $1, L_{p}^{\mathrm{Ki}}(\mathcal{T})$ modulo $\gamma^{\prime}-1$ is equal to $C_{p, 2} \times L_{p}^{\mathrm{MTT}}\left(f_{2}\right) \in \mathbb{Z}_{p}[[\Gamma]]$, where $L_{p}^{\mathrm{MTT}}\left(f_{2}\right) \in \mathbb{Z}_{p}[[\Gamma]]$ is the $p$-adic $L$ function by Mazur, Tate and Teitelbaum [MTT86]. Since $C_{p, 2}$ is a $p$-adic unit by Proposition 6.4, it suffices to prove the following claim to have the equality of the IMC for $\mathcal{T}$. 


\section{ON THE TWO-VARIABLE IWASAWA MAIN CONJECTURE}

Claim 9.12. We have the equality $\left(L_{p}^{\mathrm{MTT}}\left(f_{2}\right)\right)=(\gamma-\chi(\gamma))$ in $\mathbb{Z}_{p}[[\Gamma]]$.

Proof. We denote by $g \in \mathbb{Z}_{p}[[\Gamma]]$ the quotient $L_{p}^{\mathrm{MTT}}\left(f_{2}\right) /(\gamma-\chi(\gamma))$. We would like to show that $g$ is a unit in $\mathbb{Z}_{p}[[\Gamma]]$. For any element $h \in \mathbb{Z}_{p}[[\Gamma]]$, we regard $h$ to be the function on $\mathbb{Z}_{p}$ by setting $h(s)=\chi^{s}(h)$ for $s \in \mathbb{Z}_{p}$. The trivial zero conjecture [MTT86], which was already proved by Greenberg and Stevens [GS93], gives us an equality as follows:

$$
\left.L_{p}^{\mathrm{MTT}}\left(f_{2}\right)(s)^{\prime}\right|_{s=1}=\chi(\gamma) \log _{p}(\chi(\gamma)) \times\left. g(s)\right|_{s=1}=\mathcal{L}_{p} \times \frac{L\left(f_{2}, 1\right)}{\Omega_{\infty, 2}^{+}},
$$

where $\mathcal{L}_{p} \in \mathbb{Q}_{p}$ is the $L$-invariant defined to be $\log _{p}(q) / \operatorname{ord}_{p}(q)$ by using the Tate period $q$ for the Tate curve $X_{0}(11) / \mathbb{Q}_{p}$. By numerical calculation, we have $\operatorname{ord}_{p}\left(\mathcal{L}_{p}\right)=1=\operatorname{ord}_{p}\left(\chi(\gamma) \log _{p}(\chi(\gamma))\right)$ for $X_{0}(11)$ (cf. [MTT86, §13]). Consequently, $\left.g(s)\right|_{s=1} \in \mathbb{Z}_{p}$ is a unit. By the Weierstrass preparation theorem, $g \in \mathbb{Z}_{p}[[\Gamma]]$ must be a unit. This completes the proof of Claim 9.12.

\section{ACKNowledgements}

The author would like to thank Ralph Greenberg, Yoshitaka Hachimori and Kazuo Matsuno for stimulating discussion. He is also grateful to the anonymous referee who gave suggestions on the construction of the paper to make it more understandable.

\section{REFERENCES}

BK90 S. Bloch and K. Kato, L-functions and Tamagawa numbers of motives, in The Grothendieck Festschrift I, Progr. Math. 86 (1990), 333-400.

Bou85 N. Bourbaki, Eléments de mathématique, in Algèbre commutative (Masson, Paris, 1985), Chapters $5-7$.

CP89 J. Coates and B. Perrin-Riou, On p-adic L-functions attached to motives over Q, Adv. Stud. Pure Math. 17 (1989), 23-54.

Del69 P. Deligne, Formes modulaires et représentations l-adiques, in Séminaires Bourbaki no. 355, Lecture Notes in Mathematics, vol. 179 (Springer, Berlin, 1969), 139-172.

Del79 P. Deligne, Valeurs de fonctions L et périodes d'intégrales, in Automorphic forms, representations and L-functions, Proceedings of Symposia in Pure Mathematics, vol. 33(2) (American Mathematical Society, Providence, RI, 1979), 313-346.

EPW06 M. Emerton, R. Pollack and T. Weston, Variation of the Iwasawa invariants in Hida families, Invent. Math. 163 (2006), 523-580.

FC90 G. Faltings and C-L. Chai, Degeneration of abelian varieties, with an appendix by D. Mumford, Ergebnisse der Mathematik und ihrer Grenzgebiete (3), vol. 22 (Springer, Berlin, 1990).

Fon94 J. M. Fontaine (ed.), Périodes p-adiques, in Séminaire de Bures, 1988, Astérisque 223 (1994).

Fuk03 T. Fukaya, Coleman power series for $K_{2}$ and $p$-adic zeta functions of modular forms, Doc. Math. Extra Vol. (2003), 387-442.

Gre87 R. Greenberg, Iwasawa theory for p-adic representations, Adv. Stud. Pure Math. 17 (1987), 97-137.

Gre91 R. Greenberg, Iwasawa theory for motives, London Mathematical Society Lecture Note Series, vol. 153 (Cambridge University Press, Cambridge, 1991), 211-234.

Gre94 R. Greenberg, Iwasawa theory for p-adic deformations of motives, Proceedings of Symposia in Pure Mathematics, vol. 55(2) (American Mathematical Society, Providence, RI, 1994), 193-223.

Gre99 R. Greenberg, Iwasawa theory for elliptic curves, in Arithmetic theory of elliptic curves, Lecture Notes in Mathematics, vol. 1716 (Springer, Berlin, 1999), 51-144.

GS93 R. Greenberg and G. Stevens, $p$-adic L-functions and p-adic periods of modular forms, Invent. Math. 111 (1993), 407-447. 


\section{ON THE TWO-VARIABLE IWASAWA MAIN CONJECTURE}

GV00 R. Greenberg and V. Vatsal, On the Iwasawa invariants of elliptic curves, Invent. Math. 142 (2000), 17-63.

Hid86a H. Hida, Iwasawa modules attached to congruences of cusp forms, Ann. Sci. École Norm. Sup. (4) 19 (1986), 231-273.

Hid86b H. Hida, Galois representations into $\mathrm{GL}_{2}\left(\mathbb{Z}_{p}[[X]]\right)$ attached to ordinary cusp forms, Invent. Math. 85 (1986), 545-613.

Hid93 H. Hida, Elementary theory of L-functions and Eisenstein series, London Mathematical Society Student Texts, vol. 26 (Cambridge University Press, Cambridge 1993).

Kat93 K. Kato, Lectures on the approach to Iwasawa theory for Hasse-Weil L-functions via B $B_{\mathrm{dR}}$. I, in Arithmetic algebraic geometry, Lecture Notes in Mathematics, vol. 1553 (Springer, Berlin, 1993), 50-163.

Kat04 K. Kato, p-adic Hodge theory and values of zeta functions of modular forms, Astérisque 295 (2004), $117-290$.

Kit94 K. Kitagawa, On standard p-adic L-functions of families of elliptic cusp forms, in p-adic monodromy and the Birch and Swinnerton-Dyer conjecture, Contemporary Mathematics, vol. 165 (American Mathematical Society, Providence, RI, 1994), 81-110.

Maz72 B. Mazur, Rational points of abelian varieties with values in towers of number fields, Invent. Math. 18 (1972), 183-266.

MTT86 B. Mazur, J. Tate and J. Teitelbaum, On p-adic analogues of the conjectures of Birch and Swinnerton-Dyer, Invent. Math. 84 (1986), 1-48.

MW86 B. Mazur and A. Wiles, On p-adic analytic families of Galois representations, Compositio Math. 59 (1986), 231-264.

NP00 J. Nekovar and A. Plater, On the parity of ranks of Selmer groups, Asian J. Math. 4 (2000), 437-497.

NSW00 J. Neukirch, A. Schmidt and K. Wingberg, Cohomology of number fields, Grundlehren der Mathematischen Wissenschaften, vol. 323 (Springer, Berlin, 2000).

OV02 Y. Ochi and O. Venjakob, On the structure of Selmer groups over p-adic Lie extensions, J. Algebraic Geom. 11 (2002), 547-580.

Och01 T. Ochiai, Control theorem for Greenberg's Selmer groups for Galois deformations, J. Number Theory 88 (2001), 59-85.

Och03 T. Ochiai, A generalization of the Coleman map for Hida deformations, Amer. J. Math. 125 (2003), 849-892.

Och05 T. Ochiai, Euler system for Galois deformation, Ann. Inst. Fourier (Grenoble) 55 (2005), 113-146.

Ogg69 A. Ogg, On the eigenvalues of Hecke operators, Math. Ann. 179 (1969), 101-108.

Rub91 K. Rubin, The 'main conjectures' of Iwasawa theory for imaginary quadratic fields, Invent. Math. 103 (1991), 25-68.

Ser72 J. P. Serre, Propriétés galoisiennes des points d'ordre fini des courbes elliptiques, Invent. Math. 15 (1972), 259-331.

Wi186 A. Wiles, On p-adic representations for totally real fields, Ann. of Math. (2) 123 (1986), 407-456.

Wil88 A. Wiles, On $\lambda$-adic representations associated to modular forms, Invent. Math. 94 (1988), 529-573.

Tadashi Ochiai ochiai@math.wani.osaka-u.ac.jp

Department of Mathematics, Osaka University, 1-16, Machikaneyama, Toyonaka, Osaka, Japan, 560-0043 